\title{
The Lerch Zeta function III. Polylogarithms and special values
}

\author{
Jeffrey C. Lagarias ${ }^{1}$ and Wen-Ching Winnie Li $\mathrm{i}^{*}$
}

*Correspondence:
wli@math.psu.edu
2Department of Mathematics,
Pennsylvania State University,
University Park, PA 16802-8401,
USA
Full list of author information is
available at the end of the article

available at the end of the article

\section{严 Springer}

\begin{abstract}
This paper studies algebraic and analytic structures associated with the Lerch zeta function, complex variables viewpoint taken in part II. The Lerch transcendent $\Phi(s, z, c):=\sum_{n=0}^{\infty} \frac{z^{n}}{(n+c)^{s}}$ is obtained from the Lerch zeta function $\zeta(s, a, c)$ by the change of variable $z=e^{2 \pi i a}$. We show that it analytically continues to a maximal domain of holomorphy in three complex variables $(s, z, c)$, as a multivalued function defined over the base manifold $\mathbb{C} \times\left(\mathbb{P}^{1}(\mathbb{C}) \backslash\{0,1, \infty\}\right) \times(\mathbb{C} \backslash \mathbb{Z})$ and compute the monodromy functions describing the multivaluedness. For positive integer values $s=m$ and $c=1$ this function is closely related to the classical $m$-th order polylogarithm $\operatorname{Lim}(z)$. We study its behavior as a function of two variables $(z, c)$ for "special values" where $s=m$ is an integer. For $m \geq 1$ we show that it is a one-parameter deformation of $L i_{m}(z)$, which satisfies a linear ODE, depending on $c \in \mathbb{C}$, of order $m+1$ of Fuchsian type on the Riemann sphere. We determine the associated $(m+1)$-dimensional monodromy representation, which is a non-algebraic deformation of the monodromy of $L i_{m}(z)$.
\end{abstract}

Mathematics Subject Classification: Primary 11M35; Secondary 33B30

\section{Contents}

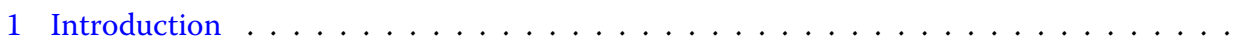

1.1 Analytic continuation in three complex variables . . . . . . . . . . . .

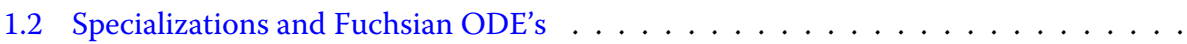

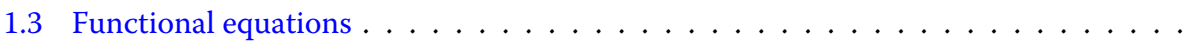

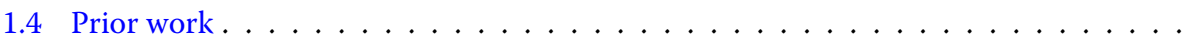

1.5 Present work

2 Summary of main results

3.1 Analytic continuation of Lerch zeta function . . . . . . . . . . . . . . .

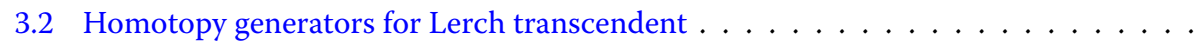

3.3 Multivalued continuation of Lerch transcendent . . . . . . . . . . . . . . .

3.4 Conditions for vanishing monodromy $\ldots \ldots \ldots \ldots \ldots$

3.5 Extended analytic continuation . . . . . . . . . . . . . . .

4 Differential-difference operators and monodromy functions . . . . . . . . . . . .

5 Specialization of Lerch transcendent: $s$ a non-positive integer $\ldots \ldots \ldots$. . . . . .

6 Double specialization: periodic zeta function $\ldots \ldots \ldots \ldots \ldots$

7 Specialization of Lerch transcendent: $s$ a positive integer . . . . . . . . . . . . . .

8 Double specialization: Deformed polylogarithm $\ldots \ldots \ldots \ldots$

๑) 2016 Lagarias and Li. This article is distributed under the terms of the Creative Commons Attribution 4.0 International License (http://creativecommons.org/licenses/by/4.0/), which permits unrestricted use, distribution, and reproduction in any medium, provided you give appropriate credit to the original author(s) and the source, provide a link to the Creative Commons license, and indicate if changes were made. 


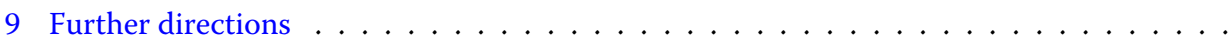

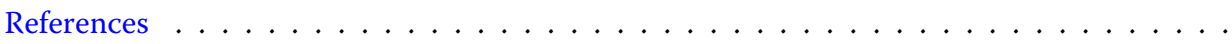

\section{Introduction}

In this paper we study the Lerch transcendent $\Phi(s, z, c)$, defined by

$$
\Phi(s, z, c):=\sum_{n=0}^{\infty} \frac{z^{n}}{(n+c)^{s}}
$$

which is obtained from the Lerch zeta function

$$
\zeta(s, a, c)=\sum_{n=0}^{\infty} e^{2 \pi i n a}(n+c)^{-s}
$$

under the change of variable $z:=e^{2 \pi i a}$. The Lerch transcendent $\Phi(s, z, c)$ is called by some authors the "Lerch zeta function" (e.g. Oberhettinger [62]), although $\zeta(s, a, c)$ is the function originally studied by Lerch [50] in 1887 . One obtains by double specialization at $z=1$ and $c=1$ the Riemann zeta function

$$
\zeta(s)=\Phi(s, 1,1)=\sum_{n=0}^{\infty} \frac{1}{(n+1)^{s}},
$$

and this expansion is valid in the half-plane $\operatorname{Re}(s)>1$.

In his 1900 problem list Hilbert [34] raised a question related to the Lerch transcendent. This question appears just after the 18-th problem, perhaps intended as a prologue to several of the subsequent problems. Hilbert remarked that functions that satisfy algebraic partial differential equations form a class of "significant functions", but that a number of important functions seem not to belong to this class. He wrote

The function of the two variables $s$ and $x$ defined by the infinite series

$$
\zeta(s, x)=x+\frac{x^{2}}{2^{s}}+\frac{x^{3}}{3^{s}}+\frac{x^{4}}{4^{s}}+\cdots
$$

which stands in close relation with the function $\zeta(s)$, probably satisfies no algebraic partial differential equation. In the investigation of this question the functional equation

$$
x \frac{\partial \zeta(s, x)}{\partial x}=\zeta(s-1, x)
$$

will have to be used.

The function $\zeta(s, x)$ is sometimes called Jonquiére's function because it was studied in 1889 by Jonquiére [36]. ${ }^{1}$ It is obtained as $\zeta(s, x)=x \Phi(s, x, 1)$, where $\Phi(s, x, 1)$ is from the specialization of the Lerch transcendent at value $c=1$. In 1920 Ostrowski [64] justified Hilbert's assertion by proving that $\zeta(s, x)$ satisfies no algebraic differential equation. Further work done on this question is discussed in Garunkštis and Laurenčikas [26].

The Lerch transcendent $\Phi(s, z, c)$, which has an extra variable $c$, circumvents Hilbert's objection and belongs to Hilbert's class of "significant functions". This comes about as

${ }^{1}$ Under the substitution $x=e^{2 \pi i a}$ it has also been called the periodic zeta function (Apostol [3, Sec.12.7]). 
follows. We introduce the two linear partial differential operators with polynomial coefficients

$$
\mathrm{D}_{\Phi}^{-}:=z \frac{\partial}{\partial z}+c, \quad \text { and } \quad \mathrm{D}_{\Phi}^{+}:=\frac{\partial}{\partial c} .
$$

One can show that the Lerch transcendent satisfies two independent ladder relations:

$$
\mathrm{D}_{\Phi}^{-} \Phi(s, z, c)=\Phi(s-1, z, c)
$$

and

$$
\mathrm{D}_{\Phi}^{+} \Phi(s, z, c)=-s \Phi(s+1, z, c)
$$

see Theorem 2.3 below. By combining these operators, one finds that the Lerch transcendent satisfies a linear partial differential equation with polynomial coefficients,

$$
\left(\mathrm{D}_{\Phi}^{-} \mathrm{D}_{\Phi}^{+}\right) \Phi(s, z, c):=\left(z \frac{\partial}{\partial z} \frac{\partial}{\partial c}+c \frac{\partial}{\partial c}\right) \Phi(s, z, c)=-s \Phi(s, z, c),
$$

so that it is a "significant function" in Hilbert's sense. We comment more on this linear PDE below.

The Lerch transcendent yields classical polylogarithms under suitable specialization of its variables (up to an inessential factor). Taking $s=m$ a positive integer, and further taking $c=1$, yields a function closely related to the $m$-th order (Euler) polylogarithm

$$
L i_{m}(z):=\sum_{n=1}^{\infty} \frac{z^{n}}{n^{m}}
$$

namely

$$
L i_{m}(z)=z \Phi(m, z, 1)
$$

We, therefore, define the function

$$
L i_{s}(z, c):=z \Phi(s, z, c)=\sum_{n=0}^{\infty} \frac{z^{n+1}}{(n+c)^{s}}
$$

and call it the extended polylogarithm. This function interpolates all polylogarithms via the parameter $s$; simultaneously it gives a deformation of the polylogarithm with deformation parameter $c$. For nonpositive integers $s=-m \leq 0$ it is known that the resulting function $L i_{-m}(z, c)$ is a rational function of the two variables $(z, c)$; we term it the negative polylogarithm of order $-m$.

The Lerch transcendent has a connection with mathematical physics. The first author will show in [45] that the Lerch zeta function has a fundamental association with the real Heisenberg group. The relation is visible in the commutation relations

$$
\mathrm{D}_{\Phi}^{+} \mathrm{D}_{\Phi}^{-}-\mathrm{D}_{\Phi}^{-} \mathrm{D}_{\Phi}^{+}=I
$$


satisfied by the operators (1.5) and (1.6). We may reformulate the linear PDE that the Lerch transcendent satisfies, using the modified operator

$$
\Delta_{\Phi}:=\frac{1}{2}\left(\mathrm{D}_{\Phi}^{+} \mathrm{D}_{\Phi}^{-}+\mathrm{D}_{\Phi}^{-} \mathrm{D}_{\Phi}^{+}\right)=z \frac{\partial}{\partial z} \frac{\partial}{\partial c}+c \frac{\partial}{\partial c}+\frac{1}{2} I .
$$

One may then rewrite the PDE (1.7) in the eigenfunction form

$$
\Delta_{\Phi} \Phi(s, z, c)=-\left(s-\frac{1}{2}\right) \Phi(s, z, c) .
$$

This linear partial differential operator formally has the $x p$-form suggested as a possible form of a Hilbert-Polya operator encoding the zeta zeros as eigenvalues (Berry and Keating $[8,9])$; more details are given in Sect. 9.

The work of this paper determines new basic analytic properties of this function, which may bring insight to its specializations such as the Riemann zeta function and the polylogarithms. We construct an analytic continuation of the Lerch transcendent in all three variables, revealing its fundamental character as a multivalued function. We give an exact determination of its multivaluedness, specified by monodromy functions, and determine the effect of this multivalued analytic continuation on the partial differential equations and difference equations above. An important feature is that this analytic continuation does not extend to certain sets over a base manifold which we term singular strata; these form the branch locus for the multivaluedness. In the $(z, c)$-variables these are points where either $c$ is a nonpositive integer and/or where $z=1$ or $z=0$. In particular this three-variable analytic continuation omits the specialization to the Riemann zeta function given in (1.3), which occurs at the singular stratum point $(z, c)=(1,1)$. The Lerch transcendent does possess additional analytic continuations in fewer variables valid on some singular strata outside the analytic continuation in three variables; for example, the Hurwitz zeta function $\zeta(s, c)=\sum_{n=0}^{\infty}(n+c)^{-s}$ arises on (one branch of) the singular stratum $z=1$ with $c$ variable. These additional analytic continuations typically include meromorphic continuations in the $s$-variable to all $s \in \mathbb{C}$. However, for many parameter ranges these functions in fewer variables are not continuous limits of the three-variable analytic continuations.

A particular goal of the paper is to understand the relation of this analytic continuation to the multivalued structure associated with the polylogarithm. The specialization to obtain the polylogarithm takes $c=1$ (and also $s=m \geq 1$, a positive integer), which lies on a singular stratum over the Lerch zeta manifold $\mathcal{M}$ in the $(a, c)$-variables described in Sect. 1.1. In part II, however, we showed that the three-variable analytic continuation has removable singularities at the points $c=n$ for $n \geq 1$ and hence extends to a larger manifold $\mathcal{M}^{\sharp}$. The polylogarithm case $c=1$ is, therefore, covered in this extended analytic continuation when projected to the manifold $\mathcal{N}^{\sharp}$ in the $(z, c)$-variables described below.

The Lerch transcendent has the new feature that its analytic continuation introduces a new singular stratum consisting of the $z=0$ manifold, which is a branch locus around which it is multivalued, whose monodromy must be determined. This singular stratum is not directly visible in the $(a, c)$ variables used in the Lerch zeta function. Upon specializing two variables to obtain the polylogarithm in the $z$-variable, we obtain a new determination of its monodromy structure, and we also obtain an interesting one-parameter deformation of the polylogarithm in the $c$-variable. 
An additional reason for interest in this $c$-deformation, apart from containing the polylogarithms, concerns the behavior of functional equations satisfied by the Lerch transcendent. A major property of the Lerch zeta function $\zeta(s, a, c)$ is that it satisfies three-term and four-term functional equations relating certain linear combinations of functions at parameter values $s$ to those at $1-s$. The functional equation of the Riemann zeta function $\zeta(s)$ and $\zeta(1-s)$ can be derived for these functional equations, proceeding by a limiting process to a singular stratum yielding functional equations for the Hurwitz zeta function (when $\operatorname{Re}(s)>0$ ) and for the periodic zeta function (when $\operatorname{Re}(s)<1$ ), and from these recovering that of the Riemann zeta function, see Apostol [3, Chap.12]. The Lerch transcendent inherits multivalued versions of these functional equations, well-defined for all non-integer values of the $c$-parameter, but they fail to extend to the parameter values corresponding to polylogarithms, as we explain in Sect. 1.3.

The following Sects. 1.1-1.5 discuss the results of this paper in general terms, and the main results are stated in detail in Sect. 2.

\subsection{Analytic continuation in three complex variables}

We establish an analytic continuation of the Lerch transcendent in three complex variables $(s, z, c)$ as a multivalued function of the variables $(z, c)$, which are entire functions of $s$. The special choice $s=n$, for $n$ a positive integer, gives a one-parameter deformation of the $n$-th order polylogarithm.

The existence of the analytic continuation follows using the results of Part II, which gave a multivalued analytic continuation of the Lerch zeta function $\zeta(s, a, c)$, to a covering manifold of the manifold

$$
\mathcal{M}=\{(s, a, c) \in \mathbb{C} \times(\mathbb{C} \backslash \mathbb{Z}) \times(\mathbb{C} \backslash \mathbb{Z})\} .
$$

That paper also gave an extended analytic continuation to a covering manifold of

$$
\mathcal{M}^{\sharp}=\left\{(s, a, c) \in \mathbb{C} \times(\mathbb{C} \backslash \mathbb{Z}) \times\left(\mathbb{C} \backslash \mathbb{Z}_{\leq 0}\right)\right\} .
$$

In this paper we lift this continuation to the Lerch transcendent $\Phi(s, z, c)$ using the multivalued inverse change of variable

$$
a=\frac{1}{2 \pi i}(\log z)
$$

to the map $z=e^{2 \pi i a}$. We choose a branch ${ }^{2}$ of the logarithm that agrees with the usual definition in the upper-half plane and on its boundary takes $\log (1)=0, \log (-1)=\pi i$. Our choice of base point on $\mathcal{M}^{\sharp}$ is $\left(s_{0}, a_{0}, c_{0}\right)=\left(\frac{1}{2}, \frac{1}{2}, \frac{1}{2}\right)$ and with this choice of branch the point $z_{0}=-1$ lifts to $a_{0}=\frac{1}{2}$. The base manifold now becomes

$$
\mathcal{N}:=\left\{(s, z, c) \in \mathbb{C} \times\left(\mathbb{P}^{1}(\mathbb{C}) \backslash\{0,1, \infty\}\right) \times(\mathbb{C} \backslash \mathbb{Z})\right\}
$$

and, in the extended analytic continuation case,

$$
\mathcal{N}^{\sharp}:=\left\{(s, z, c) \in \mathbb{C} \times\left(\mathbb{P}^{1}(\mathbb{C}) \backslash\{0,1, \infty\}\right) \times\left(\mathbb{C} \backslash \mathbb{Z}_{\leq 0}\right)\right\} .
$$

The formulas for the monodromy functions describing the multivaluedness become correspondingly more complicated than those in Part II and give a representation $\rho_{s}$ for fixed $s \in \mathbb{C}$ of the fundamental group

$$
\pi_{1}\left(\mathcal{N}, \mathbf{x}_{0}^{\prime}\right) \simeq \pi_{1}\left(\mathbb{P}^{1}(\mathbb{C}) \backslash\{0,1, \infty\}, z_{0}\right) \times \pi_{1}\left(\mathbb{C} \backslash \mathbb{Z}, c_{0}\right)
$$

${ }^{2}$ Please note the conventions on logarithms given at the end of Sect. 2. 
with specified base point $\mathbf{x}_{0}^{\prime}=\left(s_{0}, z_{0}, c_{0}\right)=\left(\frac{1}{2},-1, \frac{1}{2}\right)$, acting on a direct sum vector space spanned by the monodromy functions. ${ }^{3}$ This vector space is generally infinite dimensional, but it degenerates at values where $s$ is an integer, see Theorem 2.4.

Two main features of the analytic continuation are as follows:

(1) The Lerch transcendent becomes single-valued on a certain covering manifold of $\mathcal{N}$, which is a regular covering (i.e. Galois covering) with solvable covering group.

(2) The Lerch transcendent continues to satisfy the two independent differentialdifference Eqs. (1.5) and (1.6) on the covering manifold.

We distinguish between the regular stratum, which are all those parameter values where the analytic continuation exists, and singular strata which correspond to parameter values at which the analytic continuation gives a branch point. That is, the analytic continuation does not apply to certain (complex) codimension one singular strata of $(z, c)$ parameter values, which include all values where the Lerch zeta function formally becomes the Hurwitz zeta function or Riemann zeta function. The singular strata values are all $(z, c)$ such that either $z=0$ or $z=1$ or $c$ is a nonpositive integer, or both. On certain singular strata, continuous limiting values may exist for restricted ranges of the $s$-parameter, as illustrated in some results in Part I, e.g. [46, Theorem 2.3].

In parallel to results given in Part II, the analytic continuation above has removable singularities at positive integer values of $c$ and extends to an analytic continuation over the larger manifold $\mathcal{N}^{\sharp}$ above.

\subsection{Specializations and Fuchsian ODE's}

We study consequences of this analytic continuation for functions of fewer variables obtained by specializing the variables. These specializations include the $n$-th order polylogarithms, corresponding to a "nonsingular" specialization at $c=1$, and $s=n \geq 1$ a positive integer. The classical specializations, giving rise to the Hurwitz zeta function or Riemann zeta function, approach singular strata where the analytic continuation breaks down. Here limiting values do exist for some ranges of the singular strata parameters, and a better understanding of the nature of these degenerations seems to be of particular interest.

First, we consider specialization to the point $c=1$. This value is a "nonsingular" value for the extended analytic continuation to $\mathcal{N}^{\sharp}$. We deduce the complete multivalued analytic continuation of the extended polylogarithm. This covers the case of Hilbert's example function in the variables $(s, x)$ above. As already noted, this specialization loses the algebraic PDE property.

Second, we consider the specialization of variables that treats $s$ as a constant. This specialization retains the linear PDE property in the $(z, c)$-variables, but loses the differentialdifference equation property that depends on variation in $s$. In the case of $s=-m$ a non-positive integer, the functions are rational functions of two variables $(z, c)$, which are polynomial in the variable $c$, and which remain well-defined on certain singular strata in $c$ and $z$. For positive integers, i.e. $s=m \in \mathbb{Z}_{\geq 1}$, this specialization gives a one-parameter deformation, with deformation parameter $c$, of the classical $m$-th order polylogarithm $L i_{m}(z)$, which corresponds to taking $c=1$.

${ }^{3}$ The base point can be moved to $\mathbf{x}_{s}^{\prime}=\left(s,-1, \frac{1}{2}\right)$ since the manifold $\mathcal{N}$ has a product structure splitting off the $s$-coordinate, in which it is simply-connected. 
Third, specializing to integer values $s=m \geq 1$ and additionally specializing $c \in \mathbb{C}$ to be fixed, the specialized function $L i_{m}(z, c)$ satisfies a linear ordinary differential equation of order $m+1$ in the $z$-variable. This ODE is of Fuchsian type with regular singular points at $\{0,1, \infty\}$ for all values of the $c$-parameter; in particular this differential equation is defined for singular stratum parameter values $c \in \mathbb{Z}_{\leq 0}$. We determine the monodromy representation of the fundamental group $\pi_{1}\left(\mathbb{P}^{1} \backslash\{0,1, \infty\},-1\right)$ for this equation as a function of the deformation parameter $c$. The monodromy is unipotent for $c \in \mathbb{Z}$, is quasi-unipotent for $c \in \mathbb{Q}$, and otherwise lies in a Borel subgroup of $G L(m+1, \mathbb{C})$ but is not quasi-unipotent. A second interesting feature is that this deformation of the monodromy varies continuously on the regular stratum, but has discontinuous behavior of the monodromy representation at the singular strata values $c \in \mathbb{Z}_{\leq 0}$.

It is known that in the case $c=1$ a mixed Hodge structure can be attached to the collection of polylogarithms for all $n \geq 1$, viewed as pro-unipotent connection over $\mathbb{P}^{1} \backslash\{0,1, \infty\}$ as described in Bloch [11]. We have not addressed the question whether a mixed Hodge structure can be associated to the singular strata cases $c \in \mathbb{Z}_{\leq 0}$, where the monodromy is unipotent.

\subsection{Functional equations}

It is well known that the Lerch zeta function $\zeta(s, a, c)$ satisfies a functional equation relating parameter values $s$ and $1-s$, which can be given in an asymmetric three-term form given by Lerch [49] as

$$
\zeta(1-s, a, c)=(2 \pi)^{-s} \Gamma(s)\left\{e^{\frac{\pi i s}{2}} e^{-2 \pi i a c} \zeta(s, 1-c, a)+e^{-\frac{\pi i s}{2}} e^{2 \pi i c(1-a)} \zeta(s, c, 1-a)\right\},
$$

and called Lerch's transformation formula, cf. [46, Theorem 5.1]. It also satisfies two symmetric four-term functional equations noted by Weil [74, p. 57], discussed in Part I and Part II. To state these, define the two functions $L^{ \pm}(s, a, c)$ by

$$
L^{ \pm}(s, a, c):=\zeta(s, a, c) \pm e^{-2 \pi i a} \zeta(s, 1-a, 1-c)=\sum_{n \in \mathbb{Z}}(\operatorname{sgn}(n))^{k} \frac{e^{2 \pi i n a}}{(n+c)^{s}}
$$

in which $\pm:=(-1)^{k}$. Second, define their completions (adding archimedean Euler factors) by

$$
\hat{L}^{ \pm}(s, a, c) ;=\pi^{\frac{s+k}{2}} \Gamma\left(\frac{s+k}{2}\right) L^{ \pm}(s, a, c),
$$

with $k \in\{0,1\}$ and superscripts $\pm=(-1)^{k}$. Here [47, Theorem 2.1] states that the two symmetrized four-term functional equations

$$
\hat{L}^{+}(s, a, c)=e^{-2 \pi i a c} \hat{L}^{+}(1-s, 1-c, a)
$$

and

$$
\hat{L}^{-}(s, a, c)=i e^{-2 \pi i a c} \hat{L}^{-}(1-s, 1-c, a)
$$

hold for all $(s, a, c)$ in a simply connected domain, the fundamental polycylinder

$$
\Omega:=\{0<\operatorname{Re}(s)<1\} \times\{0<\operatorname{Re}(a)<1\} \times\{0<\operatorname{Re}(c)<1\} .
$$

Written out, the first functional equation (1.13) has four terms and states, 


$$
\begin{aligned}
\pi^{-\frac{s}{2}} & \Gamma\left(\frac{s}{2}\right)\left(\zeta(s, a, c)+e^{-2 \pi i a} \zeta(s, 1-a, 1-c)\right) \\
& =e^{-2 \pi i a c} \pi^{\frac{s-1}{2}} \Gamma\left(\frac{1-s}{2}\right)\left(\zeta(1-s, 1-c, a)+e^{2 \pi i c} \zeta(1-s, c, 1-a)\right) .
\end{aligned}
$$

These functional equations are non-local in the $(a, c)$-variables, and it is important that they leave the fundamental polycylinder $\Omega$ invariant. One can combine two four-term functional equations in such a way as to recover the three-term functional equation for the Lerch zeta function valid on the fundamental polycylinder.

The four-term functional Eqs. (1.13), (1.14) project to functional equations for the Lerch transcendent, but become complicated to state since they involve exponential and logarithmic changes of variable. If we let $z=e^{2 \pi i a}$ then we find the projected image of the fundamental polycylinder $\Omega$ to the manifold $\mathcal{N}$ is the simply-connected domain

$$
\mathcal{D}_{\mathcal{N}}:=\{0<\operatorname{Re}(s)<1\} \times\left\{z \in \mathbb{C} \backslash \mathbb{R}_{\geq 0}\right\} \times\{0<\operatorname{Re}(c)<1\} .
$$

If we set

$$
L_{\Phi}^{ \pm}(s, z, c):=\Phi(s, z, c) \pm \frac{1}{z} \Phi\left(s, \frac{1}{z}, 1-c\right),
$$

with all variables in $\mathcal{D}_{\mathcal{N}}$ and complete these two functions with appropriate archimedean Euler factors as above then we will obtain four-term functional equations with all four terms lying in $\mathcal{D}_{\mathcal{N}}$. We then obtain under analytic continuation four-term functional equations in multivalued functions of shape

$$
\hat{L}_{\Phi}^{+}(s, z, c)=z^{-c} \hat{L}_{\Phi}^{+}\left(1-s, e^{-2 \pi i c}, \frac{1}{2 \pi i} \log z\right),
$$

and

$$
\hat{L}_{\Phi}^{-}(s, z, c)=i z^{-c} \hat{L}_{\Phi}^{-}\left(1-s, e^{-2 \pi i c}, \frac{1}{2 \pi i} \log z\right),
$$

provided that correct choices are made of branches of all the multivalued functions on each side of the equation. In principle the results of this paper permit simultaneous determination of the multivaluedness of the four terms in the functional equation following paths in $\mathcal{N}$ starting from the fundamental polycylinder $\Omega$, but this paper does not carry out such a determination.

We conclude the topic of functional equations by pointing out two important issues, which remain to be resolved.

(1) The two four-term functional equations for the Lerch transcendent are well-defined on the manifold $\mathcal{N}$ but are not well-defined on the extended manifold $\mathcal{N}^{\sharp}$. The extended manifold $\mathcal{N}^{\sharp}$ glues in the integer values $c=n \geq 1$ in the Lerch transcendent $(s, z, c)$ parameters, and these extra values include exactly the value $c=1$ relevant to studying polylogarithms. This obstruction to extension occurs because always at least one of the four terms in the functional equation lies on a genuine singular stratum. A consequence is that four-term functional equations do not appear when studying the polylogarithm itself. Problems also occur with extending the three-term functional equations.

It is possible that further information can be extracted from these functional equations at the polylogarithm values, if one approaches these points along specific paths 
for restricted ranges of parameter values. In the $c$-deformation of the polylogarithm we study, the multivalued functional equations relating values at $s$ and $1-s$ "turn on" when $c$ takes a non-integer value. Perhaps some modified functional equations in fewer variables survive in the limit as a value $c=n \geq 1$ is approached for suitable ranges of the $s$-variable, because continuous limits to singular strata exist for some range of $s$, as shown in part I. One may also ask whether there is a "vanishing cycle" interpretation for some of this limit behavior.

(2) On the other hand, at the polylogarithmic points $c=m \geq 1$, new functional equations appear. Polylogarithms $L i_{m}(z)$ are well known to satisfy functional equations of quite different shape, specific to each value of $m$, sometimes relating different values of $m$ shifted by integers. These functional equations are relevant to geometry and physics, and relate these functions at different values of $z$. For the Euler dilogarithm there is a well-known functional equation found by Spence [70] in 1809, often given in the form

$L i_{2}\left(\frac{x}{1-x} \frac{y}{1-y}\right)=L i_{2}\left(\frac{x}{1-y}\right)+L i_{2}\left(\frac{y}{1-x}\right)-L i_{2}(x)-L i_{2}(y)-\log (1-x) \log (1-y)$,

see Lewin [53, Sec. 1.2]. Since $L i_{1}(x)=-\log (1-x)$ this functional equation relates polylogarithms with the two different $s$-parameter values $s=2$ and $s=1$. These shifts in the $s$-parameter are different from the $s$ to $1-s$ parameter shift in the four-term functional equation. The functional equation (1.17) can be transformed into the well-known 5-term functional equation for the Rogers dilogarithm, given by $L_{2}(x)=L i_{2}(x)+\frac{1}{2} \log x \log (1-x)$, which is

$$
L_{2}(x)+L_{2}(y)-L_{2}(x y)=L_{2}\left(\frac{x-x y}{1-x y}\right)+L_{2}\left(\frac{y-x y}{1-x y}\right),
$$

cf. Rogers [69], Zagier [80,81]. These particular functional equations have an important relation to three-dimensional geometry, specifically to Cheeger-Cherns-Simons invariants of hyperbolic 3-manifolds, cf. Dupont [20], Neumann [61]. The functional equations and related ones for higher polylogarithms seem specific to integer values $s=m>1$ of the $s$-parameter and are not known to survive deformation in $s$. Nonetheless, one may ask what is the fate of the functional equations of the dilogarithm under the $c$-deformation presented in this paper, which in (1.17) involve the integer values $s=2$ and $s=1$.

We remark that there are geometric generalizations of the dilogarithm to higher dimensional cases, which aim to preserve functional equations having a geometric meaning, see Gel'fand and MacPherson [27], Hain and MacPherson [32]. These higher-dimensional generalizations have more variables but seem not directly relatable to higher polylogarithms $L i_{n}(x)$.

\subsection{Prior work}

There is a long history of work on analytic continuation of the Lerch transcendent. After Lerch's 1887 work, in 1889 Jonquière [36] studied the two variable function $\zeta(s, x):=$ $\sum_{n=1}^{\infty} \frac{x^{n}}{n^{s}}$, obtaining various contour integral representations and a functional equation, with $s$ and $x$ allowed to take some complex values; this is the function considered by Hilbert [34]. In 1906, Barnes [4] studied the Lerch transcendent with some restrictions on 
its variables and noted some aspects of its multivalued nature. In the period 1900-2000, there was much further work on these functions obtaining analytic continuations in two of the variables, omitting one of either $a$ or $x$ (resp. $c$ for the Lerch zeta function) while pursuing other objectives, such as functional equations, which we pass over here.

Concerning analytic continuation in three variables, in 2000 Kanemitsu, Katsurada, and Yoshimoto [38] obtained an analytic continuation of the Lerch transcendent in three variables to a single-valued function on various large domains in $\mathbb{C}^{3}$. These authors also obtained formulas for special values at negative integers, related to those given below in $\$ 5$. They did not address the issue of further analytic continuation to a multivalued function. In 2008, Guillera and Sondow [30] also give a single-valued analytic continuation of $\Phi(s, z, c)$ for certain ranges of $(s, z, c)$, mostly restricting $c$ to be real-valued. Very recently, Costin and Garoufalidis [16] obtained a multivalued analytic continuation for the function $\zeta(x, s)$, calling it the "fractional polylogarithm" and denoting it $L i_{\alpha}(x)=\sum_{n=1}^{\infty} \frac{x^{n}}{n^{\alpha}}$ in variables $(\alpha, x)$ on a cover of $\mathbb{C} \times\left(\mathbb{P}^{1}(\mathbb{C}) \backslash\{0,1, \infty\}\right)$; such a continuation appears here as a special case of Theorem 3.6. Vepstas [72] also obtained results applicable to analytic continuation of the fractional polylogarithm.

The detailed multivalued nature of the Lerch zeta function $\zeta(s, a, c)$ itself in all variables appears to have been first worked out in Part II ([46]). We note that an old approach of Barnes [4] might be further developed to effect an analytic continuation of the Lerch transcendent in three variables.

Polylogarithms have their own independent history, as generalizations of the logarithm, and trace back to work of Euler [22], cf. [44, Sect. 2.4]. Much classical work on them is presented in the book of Lewin [52] and in the volume [54]. The appearance of the dilogarithm in many contexts in mathematics and physics is described in Zagier [80,81] and Oesterlé [63]. It appears in the computation of volumes of hyperbolic tetrahedra, and from there to define invariants of hyperbolic manifolds, related to its functional equations, see Neumann [61]. Polylogarithms appear in the theory of motives, in iterated integrals and mixed motives, see the discussion in Bloch [11] and Hain [31]. Generalized polylogarithms given by iterated integrals are considered in Minh et al. [55] and Joyner [37]. They appear in Beilinson's conjectures on special values of $L$-functions, in defining regulators ([5]), cf. Beilinson and Deligne [6], Huber and Wildeshaus [35]. Geometric versions of polylogarithms have been formulated (Goncharov [28,29], Cartier [12]), as well as an elliptic curve generalization of the polylogarithm (Beilinson and Levin [7]).

The multivaluedness of the polylogarithms encodes period data and also data on mixed Hodge structures. In addition $p$-adic and $\ell$-adic analogues of polylogarithms have been introduced and studied (Coleman [15], Besser [10], Furusho [24,25], Nakamura and Wajtkowiak [60], and Wojtkowiak [77-79]). In another direction, an exponentiated quantum deformation of the dilogarithm, the quantum dilograrithm, which satisfies a deformed functional equation, was proposed by Faddeev and Kashaev [23]) in 1994. It has since been much studied, see the survey of Kashaev and Nakanishi [40]. Certain dilogarithm identities play a role in integrable models and in conformal field theory (Nahm et al [58], Kirillov $[41,42])$. Motivic realizations of polylogarithms are discussed in Wildeshaus [76].

There has been much other work on the Lerch zeta function and Lerch transcendent, treatments of which can be found in books of Erdelyi et al [21, Sect. 1.10-1.12], Laurenčikas and Garunkštis [49], Srivastava and Choi [71, Chap. 2], Kanemitsu and Tsukada [39, Chaps. 3-5] and Chakraborthy, Kanemitsu and Tsukada [13, Chap. 3]. 


\subsection{Present work}

From the viewpoint of earlier work, the main point of this paper is to make an explicit study of the multivaluedness of the analytic continuation of the Lerch transcendent and to determine the effects of this analytic continuation on its other algebraic structures.

(i) We determine explicit formulas for the monodromy functions and their behavior under specialization. On the conceptual side, these formulas illuminate a new way in which the the non-positive integer values $s=-n \leq 0$ are "special values" of the associated functions, namely they are distinguished points in the $s$-parameter space in the sense that these are the unique values where all monodromy functions vanish identically, see Theorem 2.4 .

(ii) It is well known that the special values $\zeta(-n)(n \geq 0)$ at negative integers are rational numbers whose arithmetic properties allow $p$-adic interpolation ${ }^{4}$ which leads to the construction of $p$-adic $L$-functions. In Sect. 6 we show that at these special values $s=-n$ one can recover information from nearby nonsingular strata values $z \Phi(-n, z, c)$ (taking limits $c \rightarrow 0^{+}$) that is sufficient to interpolate $p$-adic $L$-functions; this is achieved using periodic zeta function values.

(iii) From the viewpoint of polylogarithms and iterated integrals, we show that under specialization this Lerch transcendent provides a complete set of solutions to a oneparameter Fuchsian deformation of the polylogarithm differential equation in the parameter $c$, and we determine its monodromy representation. This deformation of the polylogarithm may in future shed interesting light on its behavior.

The results of this paper suggest that further study be made of the limiting structure of functional equations in a neighborhood of the polylogarithm point $c=1$. As noted in Sect. 1.3 there are two sources of functional equations, which relate these functions for different values of the $s$-parameter. The three-term and four-term functional equations come from number theory and represent a generalization of the functional equations for the Riemann zeta function, relating functions with values $s$ and $1-s$. These functional equations break down at the integer parameter values $c=m \geq 1$. But exactly at those $c$ values these functions satisfy many additional functional equations which relate function values at various $s$-parameter values shifted by integers, some of which have geometric meaning. It seems of interest to determine how these additional functional equations deform in the $c$-parameter.

The extra variables in the Lerch transcendent potentially make visible new connections between these number-theoretic and geometric viewpoints. The variable $z$ in the Lerch transcendent, added to the Hurwitz zeta function variable $c$, gives it the property of satisfying a linear partial differential equation, together with raising and lowering operators, whose form connects to mathematical physics. On the number theory side, the Lerch transcendent may potentially yield new information about the Hurwitz zeta function and the Riemann zeta function, even though these functions live on singular strata. This potentially may occur by explicit limiting processes (for certain parameter ranges), using also regularization methods, and perhaps through analysis of the indirect influence of its monodromy. In Sect. 9 we suggest a number of other directions for further work.

${ }^{4}$ The recipe of Kubota and Leopoldt [43] permitting interpolation requires that suitable correction factors be applied to the values $\zeta(-n)$, related to the Euler factor at the prime $p$. 


\section{Summary of main results}

We obtain the analytic continuation and monodromy functions for the Lerch transcendent as a multivalued function defined on the complex 3 -fold

$$
\mathcal{N}:=\left\{(s, z, c) \in \mathbb{C} \times\left(\mathbb{P}^{1}(\mathbb{C}) \backslash\{0,1, \infty\}\right) \times(\mathbb{C} \backslash \mathbb{Z})\right\}
$$

The universal cover $\tilde{\mathcal{N}}$ of $\mathcal{N}$ can be identified with homotopy classes $[\gamma]$ of paths $\gamma$ in $\mathcal{N}$, and we refer to a point $[\gamma] \in \tilde{\mathcal{N}}$, where the curves start at the fixed base point $\mathbf{x}_{0}^{\prime}=\left(\frac{1}{2},-1, \frac{1}{2}\right)$ and end at a point $\gamma(1)=(s, z, c) \in \mathcal{N}$, lying over $(s, z, c)$. The point $[\gamma] \in \tilde{\mathcal{N}}$ is sometimes written as $(s, z, c,[\gamma])$ to stress the end point $(s, z, c)$ of $\gamma$. The universal covers for other manifolds containing $\mathcal{N}$ and for covers of the extended manifold $\mathcal{N}^{\sharp}$ are defined similarly.

Our notation used here for paths $\gamma$ generalizes the notation used in part II, which was restricted to be a loop having $\gamma(0)=\gamma(1)=\mathbf{x}_{0}=\left(\frac{1}{2}, \frac{1}{2}, \frac{1}{2}\right)$, with associated homotopy class $[\gamma] \in \pi_{1}\left(\mathcal{M}, \mathbf{x}_{0}\right)$. In part II we wrote $Z(s, a, c,[\gamma])$ to denote the function element centered at the endpoint $\gamma(1)$, with $(s, a, c)$ denoting local coordinates in a neighborhood of the endpoint of the loop $\gamma$. Reaching the point $(s, a, c)$ from $\gamma(1)$ can be thought of as following an additional path $\gamma^{\prime}$ from $\gamma^{\prime}(0)=\gamma(1)$ to $\gamma^{\prime}(1)=(s, a, c)$ that remains in a simply connected region obtained by cutting the manifold $\{(s, a, c) \in \mathbb{C} \times(\mathbb{C} \backslash \mathbb{Z}) \times(\mathbb{C} \backslash \mathbb{Z})\}$ along the lines $\{a=m+i t: t \leq 0\}$ for $m \in \mathbb{Z}$ and similarly in the $c$-variable. In this paper $\gamma$ denotes a path, to be thought of as the analogue of the composed path $\gamma \circ \gamma^{\prime}$, paths being composed left to right, as in Hatcher [33, p. 26]. Thus $\gamma$ need not be a closed path.

Theorem 2.1 (Lerch Transcendent Analytic Continuation)

(1) The Lerch transcendent $\Phi(s, z, c)$ on $\mathcal{N}$ analytically continues to a single-valued holomorphic function $\tilde{Z}([\gamma])=\tilde{Z}(s, z, c,[\gamma])$ on the universal cover $\tilde{\mathcal{N}}$ of $\mathcal{N}$.

(2) The function $\tilde{Z}(s, z, c,[\gamma])$ becomes single-valued on a two-step solvable regular (i.e. Galois) covering manifold $\tilde{\mathcal{N}}^{\text {solv }}$ of $\mathcal{N}$, which can be taken to be the manifold fixed by the second commutator subgroup of $\pi_{1}\left(\mathcal{N}, \mathbf{x}_{0}^{\prime}\right)$.

This is established in Sect. 3, where Theorems 3.4 and 3.5 give more detailed statements, which imply the result above. In particular we show that all monodromy functions vanish identically on a certain normal subgroup $\Gamma^{\prime}$ of $\pi_{1}\left(\mathcal{N}, \mathbf{x}_{0}^{\prime}\right)$ which contains the second derived subgroup (second commutator subgroup) $\left(\pi_{1}\left(\mathcal{N}, \mathbf{x}_{0}^{\prime}\right)\right)^{\prime \prime}$.

Our next result shows that the singularities at $c \in \mathbb{Z}_{\geq 1}$ are removable, giving an analytic continuation to a solvable covering of $\mathcal{N}^{\#}$, as follows (cf. Theorem 3.6).

Theorem 2.2 (Lerch Transcendent Extended Analytic Continuation)

(1) The Lerch transcendent $\Phi(s, z, c)$ analytically continues to a single-valued holomorphic function $\tilde{Z}(s, z, c,[\gamma])$ on the universal cover $\tilde{\mathcal{N}}^{\sharp}$ of the manifold

$$
\mathcal{N}^{\sharp}:=\left\{(s, z, c) \in \mathbb{C} \times\left(\mathbb{P}^{1}(\mathbb{C}) \backslash\{0,1, \infty\}\right) \times\left(\mathbb{C} \backslash \mathbb{Z}_{\leq 0}\right)\right\} .
$$

(2) The function $\tilde{Z}(s, z, c,[\gamma])$ becomes single-valued on a 2-step solvable covering manifold $\tilde{\mathcal{N}}^{\sharp \text { solv }}$ of $\mathcal{N}^{\sharp}$, which can be taken to be the covering that is fixed by the second commutator subgroup of $\pi_{1}\left(\mathcal{N}^{\sharp}, \mathbf{x}_{0}^{\prime}\right)$. 
In Sect. 4 we observe that the Lerch transcendent and its analytic continuation satisfy two differential-difference equations and a linear partial differential equation, as follows (cf. Theorem 4.1):

Theorem 2.3 (Lerch Transcendent Differential-Difference Operators)

(1) The Lerch transcendent $\Phi(s, z, c)$ satisfies the differential-difference equations:

$$
\left(z \frac{\partial}{\partial z}+c\right) \Phi(s, z, c)=\Phi(s-1, z, c),
$$

and

$$
\frac{\partial}{\partial c} \Phi(s, z, c)=-s \Phi(s+1, z, c) .
$$

These differential-difference equations are also satisfied by the analytic continuation $\tilde{Z}(s, z, c,[\gamma])$ of the Lerch transcendent $\Phi(s, z, c)$ on the universal cover $\tilde{\mathcal{N}}$.

(2) The Lerch transcendent $\Phi(s, z, c)$ satisfies the linear partial differential equation

$$
\left(z \frac{\partial}{\partial z}+c\right) \frac{\partial}{\partial c} \Phi(s, z, c)=-s \Phi(s, z, c) .
$$

The analytic continuation $\tilde{Z}(s, z, c,[\gamma])$ satisfies this equation on the universal cover $\tilde{\mathcal{N}}$ of $\mathcal{N}$.

(3) For each $[\tau] \in \pi_{1}\left(\mathcal{N}, \mathbf{x}_{0}^{\prime}\right)$ its associated monodromy function $M_{[\tau]}(\tilde{Z})(s, z, c,[\gamma])$ of the Lerch transcendent satisfies on $\tilde{\mathcal{N}}$ the two differential-difference equations and the linear partial differential equation (2.5).

As explained in Sect. 3.1, the fundamental group $\pi_{1}\left(\mathcal{N}, \mathbf{x}_{0}^{\prime}\right)$ is the product of $\pi_{1}\left(\mathbb{P}^{1}(\mathbb{C}) \backslash\{0,1, \infty\},-1\right)$, a free group on two generators $\left[Z_{0}\right]$ and $\left[Z_{1}\right]$, and $\pi_{1}\left(\mathbb{C} \backslash \mathbb{Z}, \frac{1}{2}\right)$, a free group on generators $\left[Y_{n}\right]$ for $n \in \mathbb{Z}$. We next determine the structure of the vector spaces $\mathcal{W}_{s}$, for fixed $s \in \mathbb{C}$, spanned by all the branches of the multivalued analytic continuation of the Lerch transcendent, over a neighborhood of a given point $(s, z, c) \in \mathcal{N}$, specifying a set of generators for these spaces. We define the space $\mathcal{W}_{s}$ to be a (generally infinite) direct sum of one-dimensional vector spaces given by particular monodromy generators, see Sect. 4. There is a generic basis for $s \notin \mathbb{Z}$ and for $s \in \mathbb{Z}$ there are linear relations among the generators, effectively reducing their number. The following result is established as Theorem 4.3:

Theorem 2.4 (Lerch Transcendent Monodromy Space) The Lerch transcendent monodromy space $\mathcal{W}_{s}$ at $s$ depends on the parameter $s \in \mathbb{C}$ as follows.

(i) (Generic case) If $s \notin \mathbb{Z}$, then $\mathcal{W}_{s}$ is an infinite-dimensional vector space and has as a basis the set of functions

$$
\left\{M_{\left[Z_{0}\right]^{-k}\left[Z_{1}\right]\left[Z_{0}\right]^{k}}(\tilde{Z}): k \in \mathbb{Z}\right\} \cup\left\{M_{\left[Y_{n}\right]}^{s}(\tilde{Z}): n \in \mathbb{Z}\right\} \cup\left\{\tilde{Z}^{s}\right\}
$$

(ii) If $s=m \in \mathbb{Z}_{>0}$, then $\mathcal{W}_{m}$ is an infinite-dimensional vector space and has as a basis the set of functions

$$
\left\{M_{\left[Z_{0}\right]^{-k}\left[Z_{1}\right]\left[Z_{0}\right]^{k}}^{m}(\tilde{Z}): k \in \mathbb{Z}\right\} \cup\left\{\tilde{Z}^{s}\right\}
$$


(iii) If $s=-m \in \mathbb{Z}_{\leq 0}$, then all Lerch transcendent monodromy functions vanish identically, i.e.

$$
M_{[\tau]}^{-m}(\tilde{Z})=0 \text { for all }[\tau] \in \pi_{1}\left(\mathcal{N}, \mathbf{x}_{0}^{\prime}\right) .
$$

Thus $\mathcal{W}_{-m}=\mathbb{C} \tilde{Z}^{s}$ is a one-dimensional vector space.

In Sects. 5-8 we specialize the Lerch transcendent variables $(s, z, c)$ to cases where $s=m$ is an integer. These are exactly the cases where the monodromy functions satisfy "non-generic" linear relations. We show that as functions of the two complex variables $(z, c)$ we obtain further analytic continuation into the singular strata of the three-variable analytic continuation given in Sect. 3. Here the singular strata correspond to $z=0,1$ and/or $c \in \mathbb{Z}_{\leq 0}$. For convenience we state results in terms of the extended polylogarithm $L i_{m}(c, z)=z \Phi(m, c, z)$.

In Sect. 5 we treat the case where $s=-m \leq 0$ is a non-positive integer. Here we may note that $L i_{-m}(c, z)$ satisfies the ordinary differential equation of order $m+1$ in the $c$-variable

$$
\frac{d^{m+1}}{d c^{m+1}} L i_{-m}(c, z)=0,
$$

which is independent of $z$. It implies that $L i_{-} m(c, z)$ is necessarily a polynomial in $c$ of degree at most $m$, having coefficients which are functions of $z$. It is known that these coefficients are rational functions of $z$; this follows from the observation of Apostol [2] in 1951 that the function $L i_{-m}(z, c)$ extends to a rational function in the $(z, c)$ variables. Here we determine formulas for these rational functions using the differential equation, as follows (Theorem 5.1).

Theorem 2.5 (c-Deformed Negative Polylogarithms) For $s=-m \in \mathbb{Z}_{\leq 0}$ the function $L i_{-m}(z, c)$ analytically continues to a rational function of $z$ and $c$ on $\mathbb{P}^{1}(\mathbb{C}) \times \mathbb{P}^{1}(\mathbb{C})$. Here $L i_{0}(z, c)=z q_{0}(z)$ and

$$
L i_{-m}(z, c)=z\left(\sum_{k=0}^{m}\left(\begin{array}{l}
m \\
k
\end{array}\right) c^{k} q_{m-k}(z)\right), \text { for } m \geq 1,
$$

in which the $q_{m}(z)$ are rational functions of $z$ given by $q_{0}(z)=\frac{1}{1-z}$ and

$$
q_{m+1}(z)=z \frac{d}{d z}\left(q_{m}(z)\right) \text { for } m \geq 0 .
$$

We also determine recursion relations for these rational functions and show they have a reflection symmetry $z^{m+1} r_{m}\left(\frac{1}{z}\right)=r_{m}(z)$. (Theorem 5.3). The rational function $L i_{-m}(c, z)$ takes well-defined values on the Riemann sphere for all $(z, c) \in \mathbb{P}^{1}(\mathbb{C}) \times \mathbb{P}^{1}(\mathbb{C})$ and thus extends to the singular strata regions given by the complex hyperplanes $c \in \mathbb{Z}_{\leq 0}$, resp. $z=0$. On the singular stratum $z=0$ these functions take finite values, but on the singular stratum $z=1$, they have a nontrivial polar part and take the constant value $\infty \in \mathbb{P}^{1}(\mathbb{C})$.

In Sect. 6 we consider the double specialization when $s=-m \leq 0$ is a nonpositive integer and $c=0$. We show the function then agrees with the analytically continued value of the periodic zeta function $F(a, s):=\sum_{n=1}^{\infty} e^{2 \pi i n a} n^{-s}$, when $z=e^{2 \pi i a}$ lies on the unit circle (Theorem 6.1). 
Theorem 2.6 (Periodic Zeta Function Special Values)

(1) For $z \in \mathbb{P}^{1}(\mathbb{C}) \backslash\{0,1, \infty\}$ and $s=-m \in \mathbb{Z}_{\leq 0}$ there holds

$$
L i_{-m}(z, 0)=\lim _{c \rightarrow 0^{+}} L i_{-m}(z, c)=\lim _{c \rightarrow 0^{+}} z \Phi(-m, z, c),
$$

where the limit is taken through values of $c$ in $0<\Re(c)<1$.

(2) For $0<\Re(a)<1$ the periodic zeta function $F(a, s)=\sum_{n=1}^{\infty} \frac{e^{2 \pi i n a}}{n^{s}}$ analytically continues to an entire function of s. In particular, for $s=-m \in \mathbb{Z}_{\leq 0}$ there holds

$$
F(a,-m)=e^{-2 \pi i a} L i_{-m}\left(e^{2 \pi i a}, 0\right)=q_{m}\left(e^{2 \pi i a}\right) .
$$

This equality (2.12) is non-trivial because it involves a limiting procedure, since the point $c=0$ lies in a singular stratum of the analytic continuation of the Lerch zeta function given in part II. This equality permits one to construct $p$-adic L-functions by interpolation from values of the Lerch zeta function. In contrast, it appears that one cannot recover values of the Hurwitz zeta function at $s=-m$ directly from the Lerch transcendent by such a limiting procedure, letting $a \rightarrow 0^{+}$or $a \rightarrow 1^{-}$; these limits do not exist.

In Sect. 7 we treat the one-variable specialization where $s=m \geq 1$ is a positive integer. We state results in terms of the function of two variables $L i_{m}(z, c)$, observing that it satisfies the (slightly different) linear PDE

$$
\left(z \frac{\partial}{\partial z} \frac{\partial}{\partial c}+(c-1) \frac{\partial}{\partial c}\right) L i_{m}(z, c)=-m L i_{m}(z, c) .
$$

We obtain the following result as Theorem 7.1.

Theorem 2.7 (c-Deformed Polylogarithm Analytic Continuation) For each positive integer $s=m \geq 1$ the function $L_{m}(z, c)$ has a meromorphic continuation in two variables $(z, c)$ to the universal cover of $\left(\mathbb{P}^{1}(\mathbb{C}) \backslash\{0,1, \infty\}\right) \times \mathbb{C}$. For fixed $\tilde{z}$ on the universal cover, this function is meromorphic as a function of $c \in \mathbb{C}$, with its singularities consisting of poles of exact order $m$ at each of the points $c \in \mathbb{Z}_{\leq 0}$.

This result gives an analytic continuation of $L i_{m}(z, c)$ to negative integer $c$, which are points that fall in the singular strata outside of the analytic continuation given in Sect. 3 .

In Sect. 8 we consider the double specialization, in which $s=m \geq 1$ is a positive integer and $c$ is fixed. Here we consider the functions $L i_{m}(z, c)$ of one variable $z$ and show they have monodromy functions which are a deformation of the monodromy of the polylogarithm $L i_{m}(z)$, viewing $c$ as a deformation parameter, with $c=1$ giving the polylogarithm, this value of $c$ is inside the three-variable analytic continuation given in $\$ 3$. Namely we observe that $F(z):=L i_{m}(z, c)$ satisfies an ordinary differential equation $D_{m+1}^{c} F(z)=0$, where

$$
D_{m+1}^{c}=z^{2} \frac{d}{\mathrm{~d} z}\left(\frac{1-z}{z}\right)\left(z \frac{d}{\mathrm{~d} z}+c-1\right)^{m} \in \mathbb{C}\left[z, \frac{d}{\mathrm{~d} z}\right] .
$$

In Theorem 8.1 we obtain the following result:

Theorem 2.8 (c-Deformed Polylogarithm Ordinary Differential Equation) Let $m \in \mathbb{Z}_{\geq 0}$ and let $c \in \mathbb{C}$ be fixed. 
(1) The function $F(z)=L i_{m}(z, c)$ satisfies the ordinary differential equation

$$
D_{m+1}^{c} F(z)=0,
$$

where $D_{m+1}^{c} \in \mathbb{C}\left[z, \frac{d}{\mathrm{~d} z}\right]$ is the linear ordinary differential operator

$$
D_{m+1}^{c}:=z^{2} \frac{d}{\mathrm{~d} z}\left(\frac{1-z}{z}\right)\left(z \frac{d}{\mathrm{~d} z}+c-1\right)^{m}
$$

of order $m+1$.

(2) The operator $D_{m+1}^{c}$ is a Fuchsian operator for all $c \in \mathbb{C}$. For each $c \in \mathbb{C}$ its singular points on the Riemann sphere are all regular and are contained in the set $\{0,1, \infty\}$.

(3) A basis of solutions of $D_{m+1}^{c}$ for $c \in \mathbb{C} \backslash \mathbb{Z}_{\leq 0}$ is, for $z \in \mathbb{C} \backslash\{(-\infty, 0] \cup[1, \infty)\}$, given by

$$
\mathcal{B}_{m+1, c}:=\left\{L i_{m}(z, c), z^{1-c}(\log z)^{m-1}, z^{1-c}(\log z)^{m-2}, \ldots, z^{1-c}\right\}
$$

(4) A basis of solutions of $D_{m+1}^{c}$ for $c=-k \in \mathbb{Z}_{\leq 0}$ is, for $z \in \mathbb{C} \backslash\{(-\infty, 0] \cup[1, \infty)\}$, given by

$$
\mathcal{B}_{m+1, c}^{*}:=\left\{L i_{m}^{*}(z,-k), z^{1-c}(\log z)^{m-1}, z^{1-c}(\log z)^{m-2}, \ldots, z^{1-c}\right\}
$$

in which

$$
L i_{m}^{*}(z,-k):=\sum_{\substack{n=0 \\ n \neq k}}^{\infty} \frac{z^{n+1}}{(n-k)^{m}}+\frac{1}{m !} z^{k+1}(\log z)^{m} .
$$

We next study the monodromy representation of the fundamental group $\pi_{1}\left(\mathbb{P}^{1}(\mathbb{C}) \backslash\{0,1, \infty\},-1\right)=\left\langle\left[Z_{0}\right],\left[Z_{1}\right]\right\rangle$ on the multivalued solutions of this differential equation. The associated monodromy representation is finite-dimensional, of dimension $m+1$, and independent of $c \in \mathbb{C}$. We show that the image of the monodromy representation lies in a Borel subgroup of $G L(m+1, \mathbb{C})$ and lies in a unipotent subgroup exactly when $c \in \mathbb{Z}$. The result splits into two cases, one for the "non-singular" strata values of $c$ and the other for the singular strata values $c \in \mathbb{Z}_{\leq 0}$. This first case is given in Theorem 8.4, as follows:

Theorem 2.9 (c-Deformed Polylogarithm Monodromy-Nonsingular Case) For each integer $s=m \geq 1$ and each $c \in \mathbb{C} \backslash \mathbb{Z}_{\leq 0}$, the monodromy action for $D_{m}^{c}$ of $\pi_{1}\left(\mathbb{P}^{1}(\mathbb{C}) \backslash\{0,1, \infty\},-1\right)=\left\langle\left[Z_{0}\right],\left[Z_{1}\right]\right\rangle$ acting on the basis

$$
\mathcal{B}_{m+1, c}:=\left\{L i_{m}(z, c), z^{1-c}(\log z)^{m-1}, z^{1-c}(\log z)^{m-2}, \ldots, z^{1-c}\right\}
$$

is given by

$$
\rho_{m, c}\left(\left[Z_{0}\right]\right):=\left(\begin{array}{cccccc}
1 & 0 & 0 & \cdots & 0 & 0 \\
0 & e^{-2 \pi i c} & \frac{2 \pi i}{1 !} e^{-2 \pi i c} & \cdots & \frac{(2 \pi i)^{m-2}}{(m-2) !} e^{-2 \pi i c} & \frac{(2 \pi i)^{m-1}}{(m-1) !} e^{-2 \pi i c} \\
\vdots & \vdots & \vdots & & \vdots & \vdots \\
0 & 0 & 0 & \cdots & e^{-2 \pi i c} & \frac{2 \pi i}{1 !} e^{-2 \pi i c} \\
0 & 0 & 0 & \cdots & 0 & e^{-2 \pi i c}
\end{array}\right)
$$


and

$$
\rho_{m, c}\left(\left[Z_{1}\right]\right):=\left(\begin{array}{cccccc}
1 & -2 \pi i & 0 & \cdots & 0 & 0 \\
0 & 1 & 0 & \cdots & 0 & 0 \\
\vdots & \vdots & \vdots & \vdots & \vdots & \vdots \\
0 & 0 & 0 & \cdots & 1 & 0 \\
0 & 0 & 0 & \cdots & 0 & 1
\end{array}\right) .
$$

The image of $\rho_{m, c}$ falls in a Borel subgroup of $G L(m+1, \mathbb{C})$. The image is unipotent if $c \in \mathbb{Z}_{>1}$ and is quasi-unipotent if $c \in \mathbb{Q} \backslash \mathbb{Z}$.

The special case $c=1$ of this result corresponds to the polylogarithm case considered by Ramakrishnan [66-68]. (We remark at the end of Sect. 8 on the issue of reconciling our formulas with those of Ramakrishnan.)

We also obtain the monodromy in the singular strata cases $c \in \mathbb{Z}_{\leq 0}$, outside the analytic continuation in Sect. 3. Here the monodromy representation exhibits a discontinuous jump from the "non-singular" strata values that seems unresolvable by a change of basis of $\left\langle\left[Z_{0}\right],\left[Z_{1}\right]\right\rangle$ (cf. Theorem 8.5).

Theorem 2.10 (c-Deformed Polylogarithm Monodromy-Singular Case) For each integer $s=m \geq 1$ and each $c \in \mathbb{Z}_{\leq 0}$, the monodromy action for $D_{m}^{c}$ of the homotopy group $\pi_{1}\left(\mathbb{P}^{1}(\mathbb{C}) \backslash\{0,1, \infty\},-1\right)=\left\langle\left[Z_{0}\right],\left[Z_{1}\right]\right\rangle$ acting on the basis

$$
\mathcal{B}_{m+1, c}^{*}:=\left\{L i_{m}^{*}(z, c), z^{1-c}(\log z)^{m-1}, z^{1-c}(\log z)^{m-2}, \ldots, z^{1-c}\right\}
$$

is given by

$$
\rho_{m, c}\left(\left[Z_{0}\right]\right):=\left(\begin{array}{cccccc}
1 & \frac{2 \pi i}{1 !} & \frac{(2 \pi i)^{2}}{2 !} & \ldots & \frac{(2 \pi i)^{m-1}}{(m-1) !} & \frac{(2 \pi i)^{m}}{m !} \\
0 & 1 & \frac{2 \pi i}{1 !} & \ldots & \frac{(2 \pi i)^{m-2}}{(m-2) !} & \frac{(2 \pi i)^{m-1}}{(m-1) !} \\
\vdots & \vdots & \vdots & & \vdots & \vdots \\
0 & 0 & 0 & \ldots & 1 & \frac{2 \pi i}{1 !} \\
0 & 0 & 0 & \ldots & 0 & 1
\end{array}\right)
$$

and

$$
\rho_{m, c}\left(\left[Z_{1}\right]\right):=\left(\begin{array}{cccccc}
1 & -2 \pi i & 0 & \cdots & 0 & 0 \\
0 & 1 & 0 & \cdots & 0 & 0 \\
\vdots & \vdots & \vdots & \vdots & \vdots & \vdots \\
0 & 0 & 0 & \cdots & 1 & 0 \\
0 & 0 & 0 & \cdots & 0 & 1
\end{array}\right) .
$$

Remark on notation for logarithms We will need two different versions of the restriction of the logarithm to a single-valued function on a cut plane. The principal branch $\log u$ makes a cut on the negative real axis, and the semi-principal branch $\log u$ makes a cut on the positive real axis. In both cases the cut line is connected to the upper half-plane, so that

$$
\log 1=\log 1=0, \quad \log (-1)=\log (-1)=\pi i .
$$


The two branches differ in the values assigned to the logarithm in the lower half-plane. However if one starts from a base point in the upper half-plane of $u$, the multivalued extensions of these two functions agree. We use $\Re(s)$ and $\Im(s)$ for the real and imaginary parts of a complex variable $s$. Finally, compositions of paths are read left to right, so $g \circ h$ with $g(1)=h(0)$ means follow path $g$ and then follow path $h$, see Hatcher [32, p. 26].

\section{Analytic continuation of Lerch transcendent}

In this section we analytically continue the Lerch transcendent $\Phi(s, z, c)$ in the three complex variables $(s, z, c)$ to a multivalued function and compute its monodromy functions, using the results of part II.

\subsection{Analytic continuation of Lerch zeta function}

We recall some details of the analytic continuation of the Lerch zeta function given in part II [47]. This analytic continuation first extended the Lerch zeta function to a single-valued function of three complex variables on the extended fundamental polycylinder:

$$
\tilde{\Omega}:=\{s: s \in \mathbb{C}\} \times\{a: 0<\Re(a)<1\} \times\{c: 0<\Re(c)<1\},
$$

This region is simply connected and is invariant under the $\mathbb{Z} / 4 \mathbb{Z}$-symmetry of the functional equation $(s, a, c) \mapsto(1-s, 1-c, a)$.

To understand the multivalued structure, for fixed $s \in \mathbb{C}$ we introduce the "elementary functions"

$$
\phi_{n}^{s}(a, c)= \begin{cases}\frac{e^{-2 \pi i n a}}{(n-c)^{s}} & \text { if } n \geq 1, \\ \frac{e^{-2 \pi i n a}}{(c-n)^{s}} & \text { if } n \leq 0 .\end{cases}
$$

These functions are well-defined in the extended fundamental polycylinder, taking the principal branch $\log z$ of the $\operatorname{logarithm}$, with $w^{s}:=\exp (s \log w)$, for $\Re(w)>0$. When analytically continued in the $c$-variable, $\phi_{n}^{s}$ has a branch point of infinite order at $c=n$, which corresponds to the branch locus $\mathcal{V}(c=n):=\{(a, c): a \in \mathbb{C}, c=n\}$ inside $\mathbb{C}^{2}$.

We also introduce the "elementary functions:"

$$
\psi_{n}^{s}(a, c):= \begin{cases}e^{-2 \pi i a c} \frac{e^{2 \pi i n c}}{(n-a)^{s-1}} & \text { if } n \geq 1, \\ e^{-2 \pi i a c} \frac{e^{2 \pi i n c}}{(a-n)^{s-1}} & \text { if } n \leq 0 .\end{cases}
$$

When analytically continued in the $a$-variable, $\psi_{n}^{s}$ has a branch point of infinite order at $a=n$, which corresponds to the branch locus $\tilde{\mathcal{V}}(a=n):=\{(a, c): a=n, c \in \mathbb{C}\}$ inside $\mathbb{C}^{2}$. When we remove all the branch loci we obtain (for fixed $s \in \mathbb{C}$ ) the punctured complex surface $\{(a, c) \in(\mathbb{C} \backslash \mathbb{Z}) \times(\mathbb{C} \backslash \mathbb{Z})\}$.

The Lerch zeta function has two expansions in terms of the elementary functions. The first is

$$
\zeta(s, a, c)=\sum_{n=0}^{\infty} \phi_{-n}^{s}(a, c)=\sum_{n=-\infty}^{0} \phi_{n}^{s}(a, c) .
$$

Here the right side of this formula converges conditionally for $0<\Re(s)<1$ when $a, c$ are real with $0<a, c<1$ (and does not converge for $s$ outside this strip). We obtain a second 
expression in terms of elementary functions using the functional equation for the Lerch zeta function $Z(s, a, c,[p t])$ on $\tilde{\Omega}$, which is

$$
\zeta(s, a, c)=\sum_{n \in \mathbb{Z}} c_{n}(s) \psi_{n}^{s}(a, c)
$$

which has coefficients $c_{n}(s)$ given by

$$
c_{n}(s):=(2 \pi)^{s-1} \Gamma(1-s) \begin{cases}e^{-\frac{\pi i(1-s)}{2}} & \text { if } n \geq 1, \\ e^{\frac{\pi i(1-s)}{2}} & \text { if } n \leq 0 .\end{cases}
$$

The right side of (3.2) converges conditionally for $0<\Re(s)<1$ for real $0<a, c<1$ with $0<\Re(s)<1$. More generally the coefficients $c_{n}(s)$ are meromorphic functions, with simple poles at $s \in \mathbb{Z}_{\geq 1}$.

The multivaluedness of the Lerch zeta function is exactly that inherited from the individual multivalued members of the right sides of (3.1) and (3.2), as we now explain. To obtain the analytic continuation in three variables, we fix as base point $\mathbf{x}_{0}:=\left(\frac{1}{2}, \frac{1}{2}, \frac{1}{2}\right) \in \tilde{\Omega}$ and consider a closed path $\gamma$ starting from the base point on the base manifold $\mathcal{M}=\{(s, a, c) \in \mathbb{C} \times(\mathbb{C} \backslash \mathbb{Z}) \times(\mathbb{C} \backslash \mathbb{Z})\}$. We view the analytic continuation on the universal cover $\tilde{\mathcal{M}}$ starting from the base point. This multivalued analytic continuation defines a sequence of single-valued analytic functions $Z(s, a, c,[\gamma])$ defined on the extended fundamental polycylinder $\tilde{\Omega}$, which depend only on the homotopy class $[\gamma] \in \pi_{1}\left(\mathcal{M}, \mathbf{x}_{0}\right)$. Here the trivial loop $[p t]$ gives

$$
Z(s, a, c,[p t])=\zeta(s, a, c)
$$

The full analytic continuation outside $\tilde{\Omega}$ is obtained by extending these functions to a simply connected domain covering the whole region $\mathcal{M}$ by making a series of cuts in the $a$-plane and $c$-plane separately.

We recall from part II generators for the homotopy group $\pi_{1}\left(\mathcal{M}, \mathbf{x}_{0}\right)$. In the $a$-variable, the generators for $\pi_{1}\left(\mathbb{C} \backslash \mathbb{Z}, a=\frac{1}{2}\right)$ are $\left\{\left[X_{n}\right]: n \in \mathbb{Z}\right\}$ in which $X_{n}$ denotes a path from base point $a=\frac{1}{2}$ that lies entirely in the upper half-plane to the point $a=n+\epsilon i$, followed by a small counterclockwise oriented loop of radius $\epsilon$ around the point $a=n$, followed by return along the path. The generators $\left[X_{n}\right]$ are pictured in Fig. 1 .

In the $c$-variable, the homotopy group $\pi_{1}\left(\mathbb{C} \backslash \mathbb{Z}, c=\frac{1}{2}\right)$ has a set of generators $\left\{\left[Y_{n}\right]\right.$ : $n \in \mathbb{Z}\}$, in which $\left[Y_{n}\right]$ denotes a path from base point $c=\frac{1}{2}$ that lies entirely in the upper half-plane to the point $c=n+\epsilon i$, followed by a small counterclockwise oriented loop of

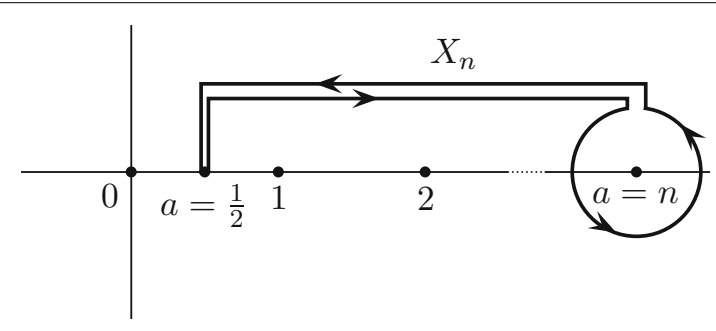

Fig. 1 Generators $\left[X_{n}\right]$ of $\pi_{1}\left(\mathbb{C} \backslash \mathbb{Z}, a=\frac{1}{2}\right)$ in the $a$-plane 


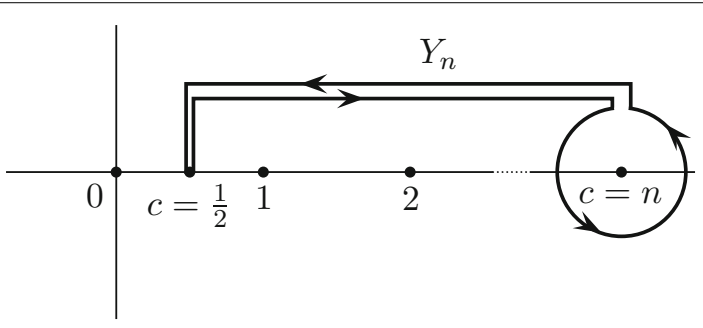

Fig. 2 Generators $\left[Y_{n}\right]$ of $\pi_{1}\left(\mathbb{C} \backslash \mathbb{Z}, c=\frac{1}{2}\right)$ in the $c$-plane

radius $\epsilon$ around the point $c=n$, followed by return along the path. The generators $\left[Y_{n}\right]$ are pictured in Fig. 2.

The monodromy of the individual elementary functions is simple. In the case of the $c=n$ singularity a counterclockwise loop traversed $k$ times in the $c$-plane (with homotopy type $\left.\left[Y_{n}\right]^{k}\right)$ sends $\phi_{n}^{s}(a, c) \mapsto e^{2 \pi i k s} \phi_{n}^{s}(a, c)$, while for the $a=n$ singularity a counterclockwise loop traversed $k$ times in the $a$-plane (corresponding to $\left[X_{n}\right]^{k}$ ) sends $\psi_{n}^{s}(a, c) \mapsto e^{2 \pi i k(1-s)} \psi_{n}^{s}(a, c)$. In part II ([47, Theorem 4.5]) we establish that the multivaluedness comes directly from the individual terms in (3.1) and (3.2). We obtain on $\tilde{\Omega}$ that

$$
Z\left(s, a, c,\left[Y_{n}\right]^{k}\right)=\zeta(s, a, c)+ \begin{cases}0 & \text { if } n \geq 1, \\ \left(e^{2 \pi i k s}-1\right) \phi_{n}^{s}(a, c) & \text { if } n \leq 0 .\end{cases}
$$

For the loops $\left[X_{n}\right]$ we obtain formulas that ${ }^{5}$ can be shown to be equivalent to

$$
Z\left(s, a, c,\left[X_{n}\right]^{k}\right)=\zeta(s, a, c)+\left(e^{-2 \pi i k s}-1\right) c_{n}(s) \psi_{n}^{s}(a, c) .
$$

Various consequences of the analytic continuation of the Lerch zeta function are deducible from (3.4) and (3.5):

(1) $Z(s, a, c,[\gamma])=\zeta(s, a, c)$ whenever $[\gamma]$ is in the commutator subgroup

$$
\mathbf{D}^{(1)}\left(\pi_{1}\left(\mathcal{M}, \mathbf{x}_{0}\right)\right):=\left[\pi_{1}\left(\mathcal{M}, \mathbf{x}_{0}\right), \pi_{1}\left(\mathcal{M}, \mathbf{x}_{0}\right)\right]
$$

of $\pi_{1}\left(\mathcal{M}, \mathbf{x}_{0}\right)([47$, Theorem 4.6]). Thus the function $Z(s, a, c,[\gamma])$ is single valued on the maximal abelian cover $\tilde{\mathcal{M}}^{a b}$ of $\mathcal{M}$. The multivaluedness on this cover is then described entirely in terms of the winding numbers $k_{n}([\gamma])\left(\right.$ resp. $\left.k_{n}^{*}([\gamma])\right)$ of the path $\gamma$ around the submanifolds $\tilde{\mathcal{V}}(a=-n)($ resp. $\mathcal{V}(c=n))$ while holding $s$ constant.

(2) For $s=-m \leq 0$ a nonpositive integer, all the monodromy functions vanish identically, i.e. for all $n, k \in \mathbb{Z}$, one has

$$
Z\left(-m, a, c,\left[X_{n}\right]^{k}\right)=Z\left(-m, a, c,\left[Y_{n}\right]^{k}\right)=\zeta(-m, a, c)
$$

([47, Theorem 7.2]).

\footnotetext{
${ }^{5}$ The formula is expressed in a different form in [48, Theorem 4.5] using gamma function identities to remove the poles
} in $c_{n}(s)$ at positive integers $s$, compare (3.33) below. 
(3) For positive integers $s=m \geq 1$ the $\left[Y_{n}\right]$-monodromy vanishes identically. However, the $\left[X_{n}\right]$ monodromy does not vanish for $s=m \geq 1$ because the functions $c_{n}(s)$ have simple poles offsetting the term $\left(e^{2 \pi i k s}-1\right)$. In fact one can show that the monodomy functions for $m \geq 1$ are

$$
Z\left(m, a, c,\left[X_{n}\right]^{k}\right)=\zeta(m, a, c)+ \begin{cases}-\frac{k}{m !} e^{-\frac{\pi i m}{2}}(2 \pi)^{m} \psi_{n}^{m}(a, c) & \text { if } m \geq 1, \\ \frac{k}{m !} e^{\frac{\pi i m}{2}}(2 \pi)^{m} \psi_{n}^{m}(a, c) & \text { if } m \leq 0 .\end{cases}
$$

The cases $s=m \geq 1$ correspond to polylogarithm parameter values.

A picture of the covering manifold structure over $\mathcal{M}$ is given in Fig. 3 in Sect. 3.2.

\subsection{Homotopy generators for Lerch transcendent}

We regard to the Lerch transcendent variables $(s, z, c)$ as lying on the manifold

$$
\mathcal{N}:=\left\{(s, z, c) \in \mathbb{C} \times\left(\mathbb{P}^{1}(\mathbb{C}) \backslash\{0,1, \infty\}\right) \times(\mathbb{C} \backslash \mathbb{Z})\right\} .
$$

which is covered by the manifold $\mathcal{M}:=\{(s, a, c) \in \mathbb{C} \times(\mathbb{C} \backslash \mathbb{Z}) \times(\mathbb{C} \backslash \mathbb{Z})\}$, with covering map $\pi^{\prime}: \mathcal{M} \rightarrow \mathcal{N}$ given by $\pi^{\prime}(s, a, c)=\left(s, e^{2 \pi i a}, c\right)$ preserving the complex-analytic structure. The covering group of deck transformations is $\mathbb{Z}$. In addition, $\mathcal{N}$ has universal cover $\tilde{\mathcal{N}}$ equal to the universal cover $\tilde{\mathcal{M}}$. Figure 3 below exhibits the set of covering maps relating $\tilde{\mathcal{M}}, \tilde{\mathcal{M}}^{a b}, \mathcal{M}$, and $\mathcal{N}$ and the associated covering groups for each factor.

To obtain an analytic continuation $\tilde{Z}$ of $\Phi(s, z, c)$ to $\tilde{\mathcal{N}}$ we use paths starting from the base point

$$
\mathbf{x}_{0}^{\prime}=(s, z, c):=\left(\frac{1}{2},-1, \frac{1}{2}\right) \in \mathcal{N},
$$

which is the image under the covering map $\pi^{\prime}\left(\mathbf{x}_{0}\right)=\mathbf{x}_{0}^{\prime}$ of the base point

$$
\mathbf{x}_{0}=(s, a, c):=\left(\frac{1}{2}, \frac{1}{2}, \frac{1}{2}\right) \in \mathcal{M}
$$

used in part II. A path $\gamma$ in $\mathcal{N}$ with basepoint $\mathbf{x}_{0}^{\prime}$ lifts via $\pi^{\prime}$ to a unique path $\tilde{\gamma}$ in $\mathcal{M}$ with basepoint $\mathbf{x}_{0}$, and we set

$$
\tilde{Z}([\gamma])=\tilde{Z}(s, z, c,[\gamma]):=Z(s, a, c,[\tilde{\gamma}]) .
$$

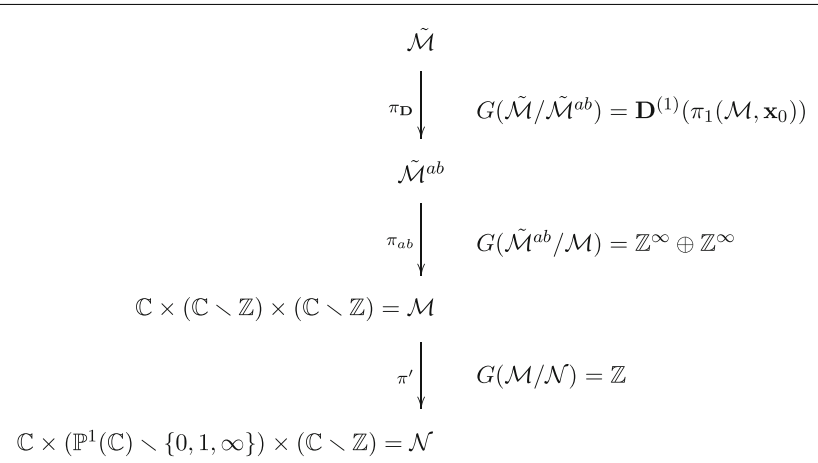

Fig. 3 Covering manifolds and automorphism groups 
This agrees with $\Phi(s, z, c)$ in a small neighborhood of $\mathbf{x}_{0}^{\prime}$ and so effects its analytic continuation to the universal cover $\tilde{\mathcal{N}} \equiv \tilde{\mathcal{M}}$. Theorem 4.2 of part II ([47]) now shows that $\tilde{Z}$ is single-valued on $\tilde{\mathcal{M}}^{a b}$.

We now specify a set of generators $\mathcal{G}^{\prime}$ of $\pi_{1}\left(\mathcal{N}, \mathbf{x}_{0}^{\prime}\right)$. The group $\pi_{1}\left(\mathcal{N}, \mathbf{x}_{0}^{\prime}\right)$ has $\pi_{1}\left(\mathcal{M}, \mathbf{x}_{0}\right)$ as a normal subgroup with quotient group $\mathbb{Z}$. In particular it contains more closed loops than $\pi_{1}\left(\mathcal{M}, \mathbf{x}_{0}\right)$, e.g. a path with endpoints $(s, a, c)$ and $(s, a+1, c)$ in $\mathcal{M}$ projects to a closed path on $\mathcal{N}$. Since $\mathcal{N}$ is a product manifold, we have

$$
\pi_{1}\left(\mathcal{N}, \mathbf{x}_{0}^{\prime}\right) \simeq \pi_{1}\left(\mathbb{P}^{1} \backslash\{0,1, \infty\},-1\right) \times \pi_{1}\left(\mathbb{C} \backslash \mathbb{Z}, \frac{1}{2}\right) .
$$

Now $\pi_{1}\left(\mathbb{P}^{1} \backslash\{0,1, \infty\},-1\right)$ is a free group on two generators $\left[Z_{0}\right]$ and $\left[Z_{1}\right]$. Our choice of loops $Z_{0}$ and $Z_{1}$ is pictured in Fig. 4.

Here we choose $Z_{0}$ to be a loop in the $z$-plane based at $z=-1$ which is a simple closed curve enclosing $z=0$ counterclockwise and not intersecting the line $L_{1}^{-}=\{1-i t: t \geq 0\}$ (so not enclosing $z=1$.) We choose $Z_{1}$ to be a simple closed curve enclosing $z=1$ counterclockwise, and not intersecting the half-line $L_{0}^{-}=\{-i t: t \geq 0\}$. The loops $Z_{0}$ and $Z_{1}$ may be viewed as closed paths in $\mathcal{N}$ which hold the variables $s=\frac{1}{2}$ and $c=\frac{1}{2}$ constant. One may also define a homotopy class $\left[Z_{\infty}\right]$ so that $\left[Z_{0}\right]\left[Z_{1}\right]\left[Z_{\infty}\right]=[$ Id $]$ is trivial. A representative of this class is a clockwise oriented loop enclosing both $z=0$ and $z=1$.

For the $c$-variable in $\mathbb{C} \backslash \mathbb{Z}$ we retain the generators $\left[Y_{n}\right]$ given in Sect. 3.1, and we obtain the full set of generators

$$
\mathcal{G}^{\prime}:=\left\{\left[Z_{0}\right],\left[Z_{1}\right]\right\} \cup\left\{\left[Y_{n}\right]: n \in \mathbb{Z}\right\}
$$

for $\pi_{1}\left(\mathcal{N}, \mathbf{x}_{0}^{\prime}\right)$.

We next determine the lifts of the loops $\left[Z_{0}\right]$ and $\left[Z_{1}\right]$ to the $a$-plane and give other relevant information on the relation of $\mathcal{M}$ and $\mathcal{N}$, as follows:

Lemma 3.1 (1) The lift $\widetilde{Z}_{0}$ to $\left(\mathcal{M}, \mathbf{x}_{0}\right)$ of the loop $Z_{0}$ on $\left(\mathcal{N}, \mathbf{x}_{0}^{\prime}\right)$ is a non-closed path with $c=s=\frac{1}{2}$ fixed and whose projection to the a-plane connects the base point $a=\frac{1}{2}$ to $a=\frac{3}{2}$ while remaining in the upper half-plane $\{a: \Im(a)>0\}$.

(2) The lift $\widetilde{Z}_{1}$ to $\left(\mathcal{M}, \mathbf{x}_{0}\right)$ of $Z_{1}$ on $\left(\mathcal{N}, \mathbf{x}_{0}^{\prime}\right)$ has $c=s=\frac{1}{2}$ fixed and is homotopic to the closed loop $X_{0}$ on $\left(\mathcal{M}, \mathbf{x}_{0}\right)$.

(3) The image group $\mathrm{H}_{1}:=\left(\pi^{\prime}\right)_{*}\left(\pi_{1}\left(\mathcal{M}, \mathbf{x}_{0}\right)\right)$ in $\pi_{1}\left(\mathcal{N}, \mathbf{x}_{0}^{\prime}\right)$ is given by

$$
\mathrm{H}_{1}:=\left\langle\left[Z_{0}\right]^{-n}\left[Z_{1}\right]\left[Z_{0}\right]^{n},\left[Y_{n}\right], \quad n \in \mathbb{Z}\right\rangle .
$$
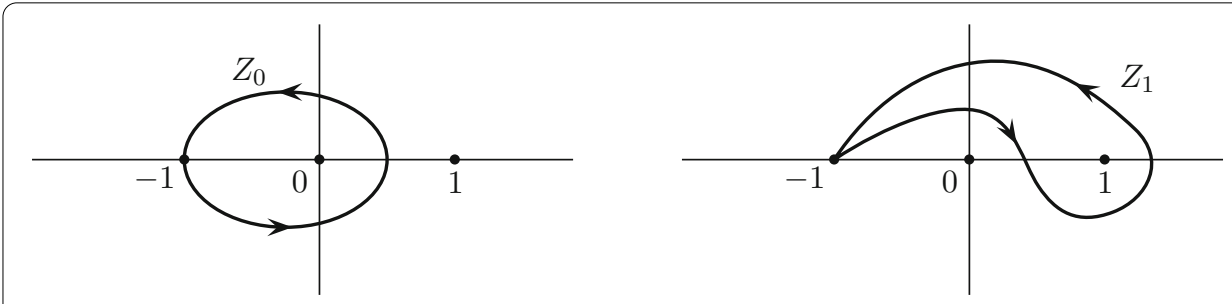

Fig. 4 Generators $\left[Z_{0}\right],\left[Z_{1}\right]$ of $\pi_{1}\left(\mathbb{P}^{1} \backslash\{0,1, \infty\},-1\right)$ in the $z$-plane 
(3) The image group $\mathrm{H}_{1}$ contains the commutator subgroup of $\pi_{1}\left(\mathcal{N}, \mathbf{x}_{0}^{\prime}\right)$, i.e.

$$
\left(\pi_{1}\left(\mathcal{N}, \mathbf{x}_{0}^{\prime}\right)\right)^{\prime}:=\left[\pi_{1}\left(\mathcal{N}, \mathbf{x}_{0}^{\prime}\right), \pi_{1}\left(\mathcal{N}, \mathbf{x}_{0}^{\prime}\right)\right] \subset \mathrm{H}_{1} .
$$

In particular, $\mathrm{H}_{1}$ is a normal subgroup of $\pi_{1}\left(\mathcal{N}, \mathbf{x}_{0}^{\prime}\right)$, and $\mathcal{M}$ is an abelian Galois covering of $\mathcal{N}$.

Proof (1) This is established by checking that images under $\pi^{\prime}$ of certain paths in the a-plane result in loops homotopic to $\left[Z_{0}\right]$ (resp. $\left.\left[Z_{1}\right]\right)$ in the $z$-plane. For $\widetilde{Z}_{0}$ we take a path from $a=\frac{1}{2}$ to the point $a=\frac{3}{2}$ that consists of a line segment in $a$-plane, from $a=\frac{1}{2}$ to $a=\frac{3}{2}$, except for a small clockwise-oriented half-circle in $\operatorname{Im}(a)>0$ centered at $a=1$, made to detour around the point $a=1$. When projected to the $z$-plane, the curve proceeds from $z=-1$ in a counterclockwise circle of radius 1 around $z=0$, with an indentation near $z=1$ to leave $z=1$ outside the loop. This image is clearly homotopic to $Z_{0}$. These are pictured in Fig. 5 .

(2) We assert that the homotopy class of the projection of the loop of $\widetilde{Z}_{1}$ pictured in Fig. 6 to the $z$-plane belongs to the homotopy class $\left[Z_{1}\right]$. To verify this, note that the path $X_{0}$ given in part II is a closed path in the $a$-plane that first moves vertically from the base point $a=\frac{1}{2}$ to $a=\frac{1}{2}+\frac{i \epsilon}{2 \pi}$, (for small enough $\epsilon$ ) then moves horizontally to $a=\frac{i \epsilon}{2 \pi}$, then moves in a counterclockwise loop of radius $\frac{\epsilon}{2 \pi}$ around $a=0$ back to $a=\frac{i \epsilon}{2 \pi}$, and finally returns to $a=\frac{1}{2}$ following the original path. One may verify that when projected to the $z$-plane, the image of $X_{0}$ moves along the $z$-axis from $z=-1$ to $z=-e^{-\epsilon}$, then proceeds in a clockwise half-circle to $z=e^{-\epsilon}$. Next, the image of the counterclockwise loop in the $a$-plane around $a=0$ is

$$
z=\exp \left(-\epsilon e^{i \theta}\right), \quad 0 \leq \theta \leq 2 \pi,
$$

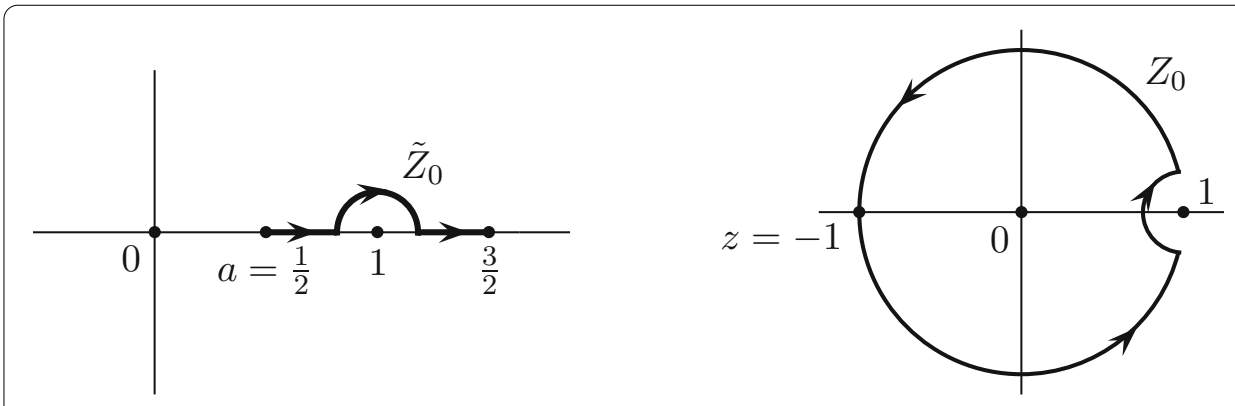

Fig. 5 Path $\tilde{Z}_{0}$ in $a$-plane and its projection to a loop $Z_{0}$ in $z$-plane
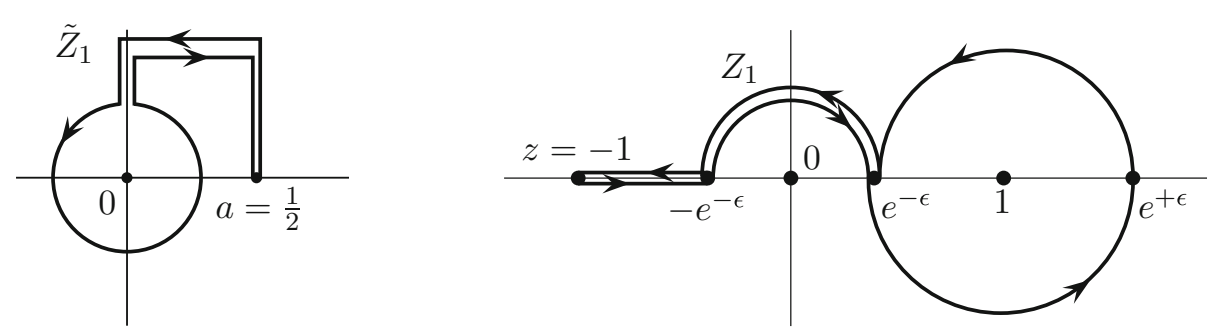

Fig. 6 Path $\tilde{Z}_{1}$ in a-plane and its projection to a loop $Z_{1}$ in $z$-plane 
which is a nearly circular path that encircles $z=1$ counterclockwise, reaching at $\theta=\pi$ the point $z=e^{\epsilon}>1$ on the real axis, with the second half of its path from $\theta=\pi$ to $\theta=2 \pi$ being the reflection of the first half in the real axis. Then it returns to $z=-1$ along the outgoing path. This path is clearly homotopic to $Z_{1}$, whence $\widetilde{\left[Z_{1}\right]}=\left[X_{0}\right]$. This is pictured in Fig. 6 .

(3) Recall that $\pi_{1}\left(\mathcal{M}, \mathbf{x}_{0}\right)$ has generating set

$$
\mathcal{G}:=\left\{\left[X_{n}\right]: n \in \mathbb{Z}\right\} \cup\left\{\left[Y_{n}\right]: n \in \mathbb{Z}\right\}
$$

in which the homotopy class $\left[X_{n}\right]$ is given by a path $X_{n}$ in the $a$-plane with basepoint $a=\frac{1}{2}$, holding $s=\frac{1}{2}, c=\frac{1}{2}$ fixed throughout, that traverses a line segment in the upper-half plane to $a=n+\epsilon i$, followed by a counterclockwise loop of radius $\epsilon$ around $a=n$, followed by return along the line segment; similarly $\left[Y_{n}\right]$ is given by a path $Y_{n}$ in the $c$-plane with basepoint $c=\frac{1}{2}$, holding $s=\frac{1}{2}$ and $a=\frac{1}{2}$ fixed throughout, that traverses a line segment in the upper-half plane to $c=n+\epsilon i$, followed by a counterclockwise loop of radius $\epsilon$ around $c=n$, followed by return along the line segment. Extending the argument of (2) we find that

$$
\left(\pi^{\prime}\right)_{*}\left(\left[X_{n}\right]\right)=\left[Z_{0}\right]^{n}\left[Z_{1}\right]\left[Z_{0}\right]^{-n}, \quad n \in \mathbb{Z}
$$

This follows since the path $\left[Z_{0}\right]^{n}\left[Z_{1}\right]$ lifted to $\mathcal{M}$ first moves from $a=\frac{1}{2}$ to the point $a=n+\frac{1}{2}$, encircles the point $a=n$ once counterclockwise and returns to $a=n+\frac{1}{2}$ and $\left[Z_{0}\right]^{-n}$, and then returns to the base point $\mathbf{x}_{0}=\left(\frac{1}{2}, \frac{1}{2}, \frac{1}{2}\right)$ in $\mathcal{M}$. We also trivially have

$$
\left(\pi^{\prime}\right)_{*}\left(\left[Y_{n}\right]\right)=\left[Y_{n}\right], \quad n \in \mathbb{Z},
$$

since the projection is constant. It follows that the image group $\mathrm{H}_{1}:=\left(\pi^{\prime}\right)_{*}\left(\pi_{1}\left(\mathcal{M}, \mathbf{x}_{0}\right)\right)$ has generating set (3.13).

(4) To verify the inclusion (3.14), note first that since both generators $\left[Z_{0}\right]$ and $\left[Z_{1}\right]$ commute with all $\left[Y_{n}\right]$ in $\pi_{1}\left(\mathcal{N}, \mathbf{x}_{0}^{\prime}\right)$, we have

$$
\left(\pi_{1}\left(\mathcal{N}, \mathbf{x}_{0}^{\prime}\right)\right)^{\prime}=\left\langle\left[Z_{0}\right]^{k}\left[Z_{1}\right]^{l}\left[Z_{0}\right]^{-k}\left[Z_{1}\right]^{-l},\left[Y_{n}\right]^{k}\left[Y_{p}\right]^{l}\left[Y_{n}\right]^{-k}\left[Y_{p}\right]^{-l}, k, l, n, p \in \mathbb{Z}\right\rangle .
$$

By (2), $\left[Z_{1}\right] \in \mathrm{H}_{1}$, whence all commutators $\left[Z_{0}\right]^{k}\left[Z_{1}\right]^{l}\left[Z_{0}\right]^{-k}\left[Z_{1}\right]^{-l}$ are in $\mathrm{H}_{1}$, and since all $\left[Y_{n}\right] \in \mathrm{H}_{1}$, we see that all generators of $\left(\pi_{1}\left(\mathcal{N}, \mathbf{x}_{0}^{\prime}\right)\right)^{\prime}$ are in $\mathrm{H}_{1}$ and the inclusion (3.14) follows.

Finally, since all subgroups of a group that contain its commutator subgroup are normal, we conclude from (3.14) that $H_{1}$ is a normal subgroup of $\pi_{1}\left(\mathcal{N}, \mathbf{x}_{0}^{\prime}\right)^{\prime}$. Thus $\mathcal{M}$ is an abelian covering of $\mathcal{N}$.

\subsection{Multivalued continuation of Lerch transcendent}

The Lerch transcendent is defined by (1.1) as an analytic function of three variables on the region

$$
\mathcal{D}_{0}=\{s: \Re(s)>1\} \times\{z:|z|<1\} \times\{c: 0<\Re(c)<1\} .
$$


on which it satisfies $\Phi\left(s, e^{2 \pi i a}, c\right)=\zeta(s, a, c)$. We now show it analytically continues to a single-valued function on the simply-connected region

$$
\mathcal{D}:=\{s: s \in \mathbb{C}\} \times\left\{z: z \in \mathbb{C} \backslash \mathbb{R}_{\geq 0}\right\} \times\{c: 0<\Re(c)<1\} \subset \mathcal{N}
$$

of $\mathcal{N}$. The region $\mathcal{D}$ is the image under the covering map $\pi^{\prime}$ of the extended fundamental polycylinder

$$
\tilde{\Omega}:=\{s: s \in \mathbb{C}\} \times\{a: 0<\mathfrak{R}(a)<1\} \times\{c: 0<\mathfrak{R}(c)<1\} \subset \mathcal{M}
$$

and the restriction $\pi^{\prime}: \tilde{\Omega} \rightarrow \mathcal{D}$ is a biholomorphic map. Theorem 2.1 of part II analytically continued $\zeta(s, a, c)$ to $\tilde{\Omega}$, which gives the analytic continuation of $\Phi(s, z, c)$ to $\mathcal{D}$, defined via

$$
\Phi\left(s, e^{2 \pi i a}, c\right)=\zeta(s, a, c) \quad \text { for }(s, a, c) \in \tilde{\Omega} .
$$

We regard $\mathcal{D}$ as embedded in the universal cover $\tilde{\mathcal{N}} \equiv \tilde{\mathcal{M}}$ by lifting it to $\tilde{\Omega}$ in $\mathcal{M}$ followed by the embedding of $\tilde{\Omega}$ in $\tilde{\mathcal{M}}$.

To describe the multivalued nature of the analytic continuation of the Lerch transcen$\operatorname{dent} \tilde{Z}$, we recall some definitions from part II.

Definition 3.2 Let $f: \tilde{\mathcal{N}} \rightarrow \mathbb{C}$ be a continuous function on the universal cover $\tilde{\mathcal{N}}$ of a manifold $\mathcal{N}$ and let $[\tau] \in \pi_{1}\left(\mathcal{N}, \mathbf{x}_{0}^{\prime}\right)$ be a homotopy class. The operator $\mathrm{Q}_{[\tau]}$ takes the function $f$ to the $[\tau]$-translated function $\mathrm{Q}_{[\tau]}(f): \tilde{\mathcal{N}} \rightarrow \mathbb{C}$ defined by

$$
\mathrm{Q}_{[\tau]}(f)([\gamma]):=f([\tau \gamma])
$$

where $\gamma$ is an arbitrary path with basepoint $\gamma(0)=\mathbf{x}_{0}^{\prime}$ and $\tau \gamma$ is the composed path (first follow $\tau$ and then $\gamma$, as in [32, p. 26]).

Definition 3.3 Let $f: \tilde{\mathcal{N}} \rightarrow \mathbb{C}$ be a continuous function and let $[\tau] \in \pi_{1}\left(\mathcal{N}, \mathbf{x}_{0}^{\prime}\right)$ be a homotopy class. The monodromy function $M_{[\tau]}(f): \tilde{\mathcal{N}} \rightarrow \mathbb{C}$ of $f$ at $[\tau]$ is defined by

$$
M_{[\tau]}(f):=\left(\mathrm{Q}_{[\tau]}-I\right)(f) .
$$

That is, for all paths $\gamma:[0,1] \rightarrow \mathcal{N}$ with base point $\gamma(0)=\mathbf{x}_{0}^{\prime}$,

$$
M_{[\tau]}(f)([\gamma]):=f([\tau \gamma])-f([\gamma]) .
$$

In the sequel we will need two different branches of the logarithm, defined as follows. We let $\log z$ denote the principal branch of the logarithm, cut along the negative real axis, with the negative real axis itself viewed as belonging to the upper half-plane, so $\log 1=0, \log (-1)=\pi i, \log (-i)=-\frac{\pi i}{2}$. The semi-principal branch $\log z$ of the logarithm is defined on the complex plane cut along the positive real axis, whose value at $z=-1$ is $\pi i$, and with the positive real axis connected to the upper half-plane, $\operatorname{so} \log (1)=0$, $\log (-1)=\pi i, \log (-i)=\frac{3 \pi i}{2}$. 
We now specify certain functions that will appear as monodromy functions of the Lerch transcendent. For each integer $n$, we now define the function

$$
f_{n}(s, z, c):=\left\{\begin{array}{cc}
e^{\pi i(s-1)} e^{2 \pi i n c} z^{-c}\left(n-\frac{1}{2 \pi i} \log z\right)^{s-1} & \text { if } n \geq 1, \\
e^{2 \pi i n c} z^{-c}\left(\frac{1}{2 \pi i} \log z-n\right)^{s-1} & \text { if } n \leq 0,
\end{array}\right.
$$

on the simply connected domain $\mathcal{D}$. Here $z^{-c}=e^{-c \log z}$, and $a=\frac{1}{2 \pi i} \log z$, but in (3.24) we evaluate the term $(a-n)^{s-1}:=e^{(s-1) \log (a-n)}$ using the principal branch of the logarithm, noting that $a-n$ (resp. $n-a)$ always has positive real part when $(s, a, c) \in \tilde{\Omega}$. The semi-principal branch $\log z$ in the formula above is needed to apply on the domain $z \in \mathbb{C} \backslash \mathbb{R}_{\geq 0}$ used in $\mathcal{D}$. The function (3.24) then extends to a function $f_{n}([\gamma])$ on $\tilde{\mathcal{N}}$ by analytic continuation.

Theorem 3.4 (Lerch Transcendent Monodromy Formulas) The Lerch transcendent $\Phi(s, z, c)$ analytically continues in three complex variables to a single-valued function $\tilde{Z}=\tilde{Z}(s, z, c,[\gamma])$ on the universal cover $\tilde{\mathcal{N}}$ of

$$
\mathcal{N}=\left\{(s, z, c) \in \mathbb{C} \times\left(\mathbb{P}^{1}(\mathbb{C}) \backslash\{0,1, \infty\}\right) \times(\mathbb{C} \backslash \mathbb{Z})\right\} .
$$

The monodromy functions of $\tilde{Z}$ for the generating set $\mathcal{G}^{\prime}$ of $\pi_{1}\left(\mathcal{N}, \mathbf{x}_{0}^{\prime}\right)$ are as follows:

(i-a) In the domain $(s, z, c) \in \mathcal{D} \subset \tilde{\mathcal{N}}$ given in (3.18) the monodromy function for the generators $\left[Z_{0}\right],\left[Z_{1}\right]$ are

$$
M_{\left[Z_{0}\right]}(\tilde{Z})(s, z, c) \equiv 0
$$

and

$$
M_{\left[Z_{1}\right]}(\tilde{Z})(s, z, c)=-\frac{(2 \pi)^{s}}{\Gamma(s)} e^{\frac{i \pi s}{2}} f_{0}(s, z, c)
$$

(i-b) On the domain $\mathcal{D}$ the functions $f_{p}(s, z, c)$ for a fixed $p \in \mathbb{Z}$ have monodromy functions

$$
M_{\left[Z_{0}\right]^{k}}\left(f_{p}\right)(s, z, c)=f_{p-k}(s, z, c)-f_{p}(s, z, c) \quad \text { for } k \in \mathbb{Z},
$$

and

$$
M_{\left[Z_{1}\right]^{k}}\left(f_{p}\right)(s, z, c) \equiv 0 \quad \text { for } k \in \mathbb{Z}
$$

In addition

$$
M_{\left[Y_{n}\right]^{k}}\left(f_{p}\right)(s, z, c) \equiv 0 \quad \text { for } k, n \in \mathbb{Z}
$$

(ii-a) On the domain $\mathcal{D}$, the monodromy functions for the generators $\left[Y_{n}\right]$ for all $n \in \mathbb{Z}$ are

$$
M_{\left[Y_{n}\right]}(\tilde{Z})(s, z, c)= \begin{cases}0 & \text { if } n \geq 1, \\ \left(e^{-2 \pi i s}-1\right) z^{-n}(c-n)^{-s} & \text { if } n \leq 0 .\end{cases}
$$


(ii-b) For a path $\gamma$ in $\mathcal{N}$ from $\mathbf{x}_{0}^{\prime}$ to an endpoint falling in the multiply-connected region

$$
\mathcal{N}_{s}=\{s\} \times\left(\mathbb{P}^{1}(\mathbb{C}) \backslash\{0,1, \infty\}\right) \times(\mathbb{C} \backslash \mathbb{Z}),
$$

there holds

$$
M_{\left[Y_{n}\right]^{-1}}(\tilde{Z})([\gamma])=-e^{2 \pi i s} M_{\left[Y_{n}\right]}(\tilde{Z})([\gamma])
$$

and

$$
M_{\left[Y_{n}\right]^{ \pm k}}(\tilde{Z})([\gamma])=\frac{e^{\mp 2 \pi i s k}-1}{e^{\mp 2 \pi i s}-1} M_{\left[Y_{n}\right]^{ \pm 1}}(\tilde{Z})([\gamma]) .
$$

Remarks. (1) The hypothesis "on the domain $\mathcal{D}$ " above means to view $(s, z, c,[\gamma]) \in \mathcal{D}$ as the endpoint of a path $\gamma$ based at $\mathbf{x}_{0}^{\prime}=\left(\frac{1}{2},-1, \frac{1}{2}\right)$ that remains entirely inside $\mathcal{D}$.

(2) The formulas given in (i-a), (i-b) above are sufficient to compute the monodromy functions for all elements of the subgroup $G_{Z}:=\left\langle\left[Z_{0}\right],\left[Z_{1}\right]\right\rangle$ of $\pi_{1}\left(\mathcal{N}, \mathbf{x}_{0}^{\prime}\right)$; these include all monodromy functions not already determined in part II.

Proof (i-a). We use the fact shown earlier that $\left(\pi^{\prime}\right)_{*}\left(\left[X_{m}\right]\right)=\left[Z_{0}\right]^{-m}\left[Z_{1}\right]\left[Z_{0}\right]^{m}$ for $m \in \mathbb{Z}$, which gives

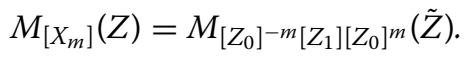

We can now apply the formula

$$
M_{\left[X_{m}\right]}(Z)(s, a, c)=\left\{\begin{array}{cc}
-\frac{(2 \pi)^{s} e^{\frac{\pi i s}{2}}}{\Gamma(s)} e^{\pi i(s-1)}(m-a)^{s-1} e^{-2 \pi i c(a-m)} & \text { if } m \geq 1, \\
-\frac{(2 \pi)^{s} e^{\frac{\pi i s}{2}}}{\Gamma(s)}(a-m)^{s-1} e^{-2 \pi i c(a-m)} & \text { if } m \leq 0,
\end{array}\right.
$$

of Theorem 4.1 of part II. This immediately gives, for $(s, z, c) \in \mathcal{D}$, on choosing $m=0$, that

$$
M_{\left[Z_{1}\right]}(\tilde{Z})(s, z, c)=-\frac{(2 \pi)^{s} e^{\frac{\pi i s}{2}}}{\Gamma(s)} z^{-c}\left(\frac{1}{2 \pi i} \log z\right)^{s-1}
$$

in which $z^{-c}=e^{-c \log z}$.

The Lerch zeta function $\zeta(s, a, c)=\sum_{n=0}^{\infty} e^{2 \pi i n a}(n+c)^{-s}$ is defined by the right side as an analytic function of three variables in the region

$$
\mathcal{U}^{+}:=\{s: \Re(s)>0\} \times\{a: \Im(a)>0\} \times\{c: \Re(c)>0\}
$$

and on this region it is invariant under $a \mapsto a+1$. Recall from part II [48, Theorem 4.1 proof] that this function analytically continues to a single-valued function in the larger domain

$$
\mathcal{O}_{1}:=\{s: \Re(s)>0\} \times\left\{a: a \in \mathcal{A}_{L}\right\} \times\{c: \Re(c)>0\},
$$

in which $\mathcal{A}_{L}=\mathbb{C} \backslash\left\{M_{k}: k \in \mathbb{Z}\right\}$ with $M_{k}=\{a=k-i t: t \geq 0\}$. We can find a lifting of the path in the class $\left[Z_{0}\right]$ in $\mathcal{M}$ that is a path in $\mathcal{O}_{1}$ from $\mathbf{x}_{0}=\left(\frac{1}{2}, \frac{1}{2}, \frac{1}{2}\right)$ to $\mathbf{x}_{1}=\left(\frac{1}{2}, \frac{3}{2}, \frac{1}{2}\right)$ in $\mathcal{M}$, which has $\Im(a)>0$ everywhere except at the endpoints, so lies in $\mathcal{U}^{+}$except at the 
endpoints. Therefore, the equality $\zeta(s, a, c)=\zeta(s, a+1, c)$ holds near both endpoints $\mathbf{x}_{0}$ and $\mathbf{x}_{1}$ of this path (in the upper half plane $\Im(a)>0$ ) and we obtain

$$
M_{\left[Z_{0}\right]}(\tilde{Z}) \equiv 0,
$$

since it is identically zero in a small open disk containing $\mathbf{x}_{0}^{\prime}$.

(i-b) The calculation of the monodromy functions of $f_{p}(s, z, c)$ for the loops $\left[Z_{0}\right]^{k},\left[Z_{1}\right]^{k}$, and $\left[Y_{n}\right]^{k}$ is straightforward, except that the case of $\left[Z_{0}\right]^{k}$ where $p+k$ and $p$ have opposite signs requires some care.

(ii-a), (ii-b) Clearly $\left(\pi^{\prime}\right)_{*}\left(\left[Y_{n}\right]\right)=\left[Y_{n}\right]$, the closed loops $\left[Y_{n}\right]^{k}$ in $\mathcal{M}$ based at $\mathbf{x}_{0}$ project to closed loops $\left[Y_{n}\right]^{k}$ based at $\mathbf{x}_{0}^{\prime}$ in $\mathcal{N}$. The monodromy formulas in Theorem 4.1(ii) of part II immediately apply to yield (3.30)-(3.32).

\subsection{Conditions for vanishing monodromy}

We study the action of the subgroups

$$
\mathrm{G}_{Z}:=\left\langle\left[Z_{0}\right],\left[Z_{1}\right]\right\rangle \text { and } \mathrm{G}_{Y}:=\left\langle\left[Y_{n}\right]: n \in \mathbb{Z}\right\rangle
$$

of $\pi\left(\mathcal{N}, \mathbf{x}_{0}^{\prime}\right)$ acting on monodromy functions. We show the actions commute and further analyze their actions to show that certain monodromy functions vanish identically.

Theorem 3.5 (Lerch Transcendent Vanishing Monodromy) Suppose that $[\tau] \in \pi_{1}\left(\mathcal{N}, \mathbf{x}_{0}^{\prime}\right)$ satisfies

$$
[\tau]:=\left[S_{1}\right]^{\epsilon_{1}} \cdots\left[S_{m}\right]^{\epsilon_{m}}
$$

with each $\left[S_{i}\right] \in\left\{\left[Z_{0}\right],\left[Z_{1}\right]\right\} \cup\left\{\left[Y_{n}\right]: n \in \mathbb{Z}\right\}$ and with each $\epsilon_{j}$ equal to one of $\{ \pm 1\}$. Define $\left[\tau_{Z}\right]$ (resp. $\left.\left[\tau_{Y}\right]\right)$ to be the element of $\pi_{1}\left(\mathcal{N}, \mathbf{x}_{0}^{\prime}\right)$ obtained by setting all generators $\left[Y_{n}\right]$ (resp. $\left[Z_{0}\right]$ and $\left.\left[Z_{1}\right]\right)$ in (3.37) to be the identity, so that

$$
\left[\tau_{Z}\right] \in \mathrm{G}_{Z}=\left\langle\left[Z_{0}\right],\left[Z_{1}\right]\right\rangle \text { and }\left[\tau_{Y}\right] \in \mathrm{G}_{Y}:=\left\langle\left[Y_{n}\right]: n \in \mathbb{Z}\right\rangle .
$$

Then the following hold:

(1) For all paths $\gamma$ based at $\mathbf{x}_{0}^{\prime}$, the monodromy function $M_{[\tau]}(\tilde{Z})([\gamma])$ satisfies

$$
M_{[\tau]}(\tilde{Z})([\gamma])=M_{\left[\tau_{Z}\right]}(\tilde{Z})([\gamma])+M_{\left[\tau_{Y}\right]}(\tilde{Z})([\gamma])
$$

(2) For all paths $\gamma$ based at $\mathbf{x}_{0}^{\prime}$, the monodromy function $M_{\left[\tau_{Y}\right]}(\tilde{Z})([\gamma])$ satisfies

$$
M_{\left[\tau_{Y}\right]}(\tilde{Z})([\gamma])=\sum_{n \in \mathbb{Z}} M_{\left[Y_{n}\right]^{k(n)}}(\tilde{Z})([\gamma]),
$$

in which $k(n) \in \mathbb{Z}$ is the sum of exponents of $\left[Y_{n}\right]$ occurring in $[\tau]$.

(3) The monodromy function $M_{[\tau]}(\tilde{Z})$ vanishes identically for any $[\tau]$ in the subgroup $\Gamma^{\prime}$ of $\pi_{1}\left(\mathcal{N}, \mathbf{x}_{0}^{\prime}\right)$ defined by

$$
\Gamma^{\prime}:=\left[\mathrm{H}_{0}: \mathrm{H}_{0}\right]
$$


in which

$$
\mathrm{H}_{0}:=\left\langle\left[Z_{0}\right]^{m}\left[Z_{1}\right]\left[Z_{0}\right]^{-m},\left[Z_{0}\right]^{m}\left[Y_{n}\right]\left[Z_{0}\right]^{-m}: m, n \in \mathbb{Z}\right\rangle .
$$

The group $\Gamma^{\prime}$ is a normal subgroup of $\left(\pi_{1}\left(\mathcal{N}, \mathbf{x}_{0}^{\prime}\right)\right)$ that contains its second commutator subgroup $\left(\pi_{1}\left(\mathcal{N}, \mathbf{x}_{0}^{\prime}\right)\right)^{\prime \prime}$. In particular its quotient $\pi_{1}\left(\mathcal{N}, \mathbf{x}_{0}^{\prime}\right) / \Gamma^{\prime}$ is a two-step solvable group.

Proof In this proof the argument is carried out for each homotopy class $[\gamma]$ separately. We regard it as fixed and so abbreviate $\left.M_{[S]}(f)[\gamma]\right)$ to $M_{[S]}(f)$, throughout.

We know that the generators $\left[Z_{0}\right]$ and $\left[Z_{1}\right]$ of $\pi_{1}\left(\mathcal{M}, \mathbf{x}_{0}^{\prime}\right)$ each commute with all generators $\left[Y_{n}\right]$, but this is not in itself sufficient to imply (3.38). The extra facts to be used are that the $\left[Z_{j}\right]$-generators annihilate all $\left[Y_{n}\right]$-monodromy functions, and the $\left[Y_{n}\right]$-generators annihilate all $\left[Z_{j}\right]$-monodromy functions. More precisely, since $z^{-n}(c-n)^{-s}$ is singlevalued in the $z$-variable for fixed $s$ and $c$ (for $n \leq 0$ ), it follows from Theorem 3.4 that

$$
M_{\left[Z_{j}\right]^{ \pm 1}}\left(M_{\left[Y_{n}\right]^{ \pm k}}(\tilde{Z})\right) \equiv 0
$$

for $j \in\{0,1\}$ and all $k, n \in \mathbb{Z}$. Also since $f_{p}(s, z, c)$ is single-valued in the $c$-variable for fixed $s$ and $z$, it follows from Theorem 3.4 that

$$
M_{\left[Y_{n}\right]^{ \pm 1}}\left(f_{p}\right) \equiv 0
$$

for all $p \in \mathbb{Z}$. These relations give for all $\left[\tau_{Y}\right] \in \mathrm{G}_{Y}$ and $\left[\tau_{Z}\right] \in \mathrm{G}_{Z}$ that

$$
\begin{aligned}
& M_{\left[Z_{j}\right]^{ \pm 1}}\left(M_{\left[\tau_{Y}\right]}(\tilde{Z})\right) \equiv 0 \quad \text { for } j \in\{0,1\} \\
& M_{\left[Y_{n}\right]^{ \pm 1}}\left(M_{\left[\tau_{Z}\right]}(\tilde{Z})\right) \equiv 0 \quad \text { for } n \in \mathbb{Z} .
\end{aligned}
$$

(1) We now prove (3.38) by induction on the length $m$ of the formula (3.37). The base case $m=1$ is clearly true. For the induction step, write $[\tau]=\left[S^{\prime}\right]\left[\tau^{\prime}\right]$, where $\left[S^{\prime}\right]=\left[S_{1}\right]^{\epsilon_{1}}$ and $\left[\tau^{\prime}\right]=\left[S_{2}\right]^{\epsilon_{2}} \ldots\left[S_{m}\right]^{\epsilon_{m}}$. Lemma 4.4 of part II gives

$$
M_{[\tau]}(\tilde{Z})=M_{\left[S^{\prime}\right]}(\tilde{Z})+M_{\left[\tau^{\prime}\right]}(\tilde{Z})+M_{\left[S^{\prime}\right]}\left(M_{\left[\tau^{\prime}\right]}(\tilde{Z})\right) .
$$

Applying the induction hypothesis to the terms on the right side gives

$$
M_{[\tau]}(\tilde{Z})=M_{\left[S_{Z}^{\prime}\right]}(\tilde{Z})+M_{\left[S_{Y}^{\prime}\right]}(\tilde{Z})+M_{\left[\tau_{Z}^{\prime}\right]}(\tilde{Z})+M_{\left[\tau_{Y}^{\prime}\right]}(\tilde{Z})+M_{\left[S^{\prime}\right]}\left(M_{\left[\tau_{Z}^{\prime}\right]}(\tilde{Z})+M_{\left[\tau_{Y}^{\prime}\right]}(\tilde{Z})\right)
$$

Next, the relations (3.42) and (3.43) imply that

$$
M_{\left[S^{\prime}\right]}\left(M_{\left[\tau_{Z}^{\prime}\right]}(\tilde{Z})+M_{\left[\tau_{Y}^{\prime}\right]}(\tilde{Z})\right)=M_{\left[S_{Y}^{\prime}\right]}\left(M_{\left[\tau_{Y}^{\prime}\right]}(\tilde{Z})\right)+M_{\left[S_{Z}^{\prime}\right]}\left(M_{\left[\tau_{Z}^{\prime}\right]}(\tilde{Z})\right)
$$

using the fact that $\left[S^{\prime}\right]$ equals one of $\left[S_{Y}^{\prime}\right]$ and $\left[S_{Z}^{\prime}\right]$ and the other term on the right side of (3.45) is identically zero since $M_{[I]}(f)=0$ for all functions $f$. Substituting (3.45) into (3.44) and applying Lemma 4.4 of part II twice to the right side yields

$$
\begin{aligned}
M_{[\tau]}(\tilde{Z}) & =M_{\left[S_{Z}^{\prime}\right]\left[\tau_{Z}^{\prime}\right]}(\tilde{Z})+M_{\left[S_{Y}^{\prime}\right]\left[\tau_{Y}^{\prime}\right]}(\tilde{Z}) \\
& =M_{\left[\tau_{Z}\right]}(\tilde{Z})+M_{\left[\tau_{Y}\right]}(\tilde{Z})
\end{aligned}
$$

which completes the induction step, yielding (3.38). 
(2) The subgroup $\mathrm{G}_{Y} \subseteq \pi_{1}\left(\mathcal{N}, \mathbf{x}_{0}^{\prime}\right)$ is the image under $\pi^{\prime}: \mathcal{M} \rightarrow \mathcal{N}$ of the subgroup $\left\langle\left[Y_{m}\right]: m \in \mathbb{Z}\right\rangle$ of $\pi_{1}\left(\mathcal{M}, \mathbf{x}_{0}\right)$. The map $\pi^{\prime}$ is injective when restricted to this subgroup; hence (3.39) is a direct consequence of the formula

$$
M_{[\tau]}(Z)=\sum_{[S] \in \mathcal{G}} M_{[S]^{k(S)}}(Z)
$$

given in Theorem 4.6 of part II when restricted to the subgroup $\mathrm{G}_{Y}$.

(3) We observe that the homomorphism from $\pi_{1}\left(\mathcal{N}, \mathbf{x}_{0}^{\prime}\right)$ to $\mathbb{Z}$ which maps $[\tau] \in$ $\pi_{1}\left(\mathcal{N}, \mathbf{x}_{0}^{\prime}\right)$ given by (3.37) to the sum of the exponents of $\left[Z_{0}\right]$ occurring in (3.37) has kernel $\mathrm{H}_{0}$ generated by

$$
\mathrm{H}_{0}:=\left\langle\left[Z_{0}\right]^{k}\left[Z_{1}\right]\left[Z_{0}\right]^{-k},\left[Z_{0}\right]^{k}\left[Y_{n}\right]\left[Z_{0}\right]^{-k}, k, n \in \mathbb{Z}\right\rangle .
$$

That is, we have the exact sequence

$$
0 \rightarrow \mathrm{H}_{0} \rightarrow \pi_{1}\left(\mathcal{N}, \mathbf{x}_{0}^{\prime}\right) \rightarrow \mathbb{Z} \rightarrow 0 .
$$

Furthermore, every element $[\tau]$ in $\pi_{1}\left(\mathcal{N}, \mathbf{x}_{0}^{\prime}\right)$ can be written as $[\tau]=[\sigma]\left[Z_{0}\right]^{k}$ for some $[\sigma]$ in $\mathrm{H}_{0}$ and $k \in \mathbb{Z}$. Note that the inclusions

$$
\left[\pi_{1}\left(\mathcal{N}, \mathbf{x}_{0}^{\prime}\right), \pi_{1}\left(\mathcal{N}, \mathbf{x}_{0}^{\prime}\right)\right] \subset \mathrm{H}_{1} \subset \mathrm{H}_{0} .
$$

follow using (3.13) and (3.14).

We next claim that the the monodromy functions $M_{[\tau]}(\tilde{Z})$ vanish identically for $[\tau] \in \Gamma^{\prime}=\left[\mathrm{H}_{0}, \mathrm{H}_{0}\right]$. To prove the claim, Theorem 4.6 of part II shows that the monodromy functions $M_{[\tau]}(Z)$ vanish identically for $[\tau]$ in the commutator subgroup $\Gamma=\left(\pi_{1}\left(\mathcal{M}, \mathbf{x}_{0}\right)\right)^{\prime}$. Consequently all monodromy functions of $\tilde{Z}$ vanish on the image group $\Gamma^{\prime}:=\left(\pi^{\prime}\right)_{*}(\Gamma)$. Now $\left(\pi^{\prime}\right)_{*}\left(\pi_{1}\left(\mathcal{M}, \mathbf{x}_{0}\right)\right)=\mathrm{H}_{0}$, so we conclude

$$
\Gamma^{\prime}=\left(\pi^{\prime}\right)_{*}(\Gamma)=\left[\mathrm{H}_{0}, \mathrm{H}_{0}\right]
$$

which is (3.40).

We next observe that $H_{0}$ is a normal subgroup of $\pi_{1}\left(\mathcal{N}, \mathbf{x}_{0}^{\prime}\right)$ because we have the inclusion of the commutator subgroup

$$
\left(\pi_{1}\left(\mathcal{N}, \mathbf{x}_{0}^{\prime}\right)\right)^{\prime} \subset \mathrm{H}_{1} \subset \mathrm{H}_{0} .
$$

It follows that $\Gamma^{\prime}=\left[\mathrm{H}_{0}, \mathrm{H}_{0}\right]$ is a normal subgroup of $\pi_{1}\left(\mathcal{N}, \mathbf{x}_{0}^{\prime}\right)$ because it is a characteristic subgroup of the normal subgroup $\mathrm{H}_{0}$ of the group (cf. Robinson [18, 1.5.6(3)]). In addition, the inclusion $\left(\pi_{1}\left(\mathcal{N}, \mathbf{x}_{0}^{\prime}\right)\right)^{\prime} \subset \mathrm{H}_{1}$ implies that

$$
\left(\pi_{1}\left(\mathcal{N}, \mathbf{x}_{0}^{\prime}\right)\right)^{\prime \prime} \subset\left[H_{1}, H_{1}\right] \subset\left[H_{0}, H_{0}\right]=\Gamma^{\prime} .
$$

It follows that $\pi_{1}\left(\mathcal{N}, \mathbf{x}_{0}^{\prime}\right) / \Gamma^{\prime}$ is solvable in two steps, with $\Gamma^{\prime} \triangleleft \mathrm{H}_{1} \triangleleft \pi_{1}\left(\mathcal{N}, \mathbf{x}_{0}^{\prime}\right)$.

The formulas in Theorem 3.5 together with those of Theorem 3.4 suffice to evaluate any monodromy function $M_{[\tau]}(\tilde{Z})$ for $[\tau] \in \pi_{1}\left(\mathcal{N}, \mathbf{x}_{0}^{\prime}\right)$. Indeed, for $[\tilde{\tau}] \in \pi_{1}\left(\mathcal{N}, \mathbf{x}_{0}^{\prime}\right)$, we 
may write $[\tilde{\tau}]=[\tau]\left[Z_{0}\right]^{n}$ for some $[\tau] \in \mathrm{H}_{0}$ and some integer $n$. Since $M_{\left[Z_{0}\right]^{n}}(\tilde{Z})=0$ by Theorem 3.4, we have

$$
M_{[\tilde{\tau}]}(\tilde{Z})=M_{[\tau]}(\tilde{Z})=M_{\left[\tau_{Z}\right]}(\tilde{Z})+M_{\left[\tau_{Y}\right]}(\tilde{Z}),
$$

where the last equality follows from (3.38). Note that $\left[\tau_{Y}\right]=\left[\tilde{\tau}_{Y}\right]$ so that $M_{\left[\tau_{Y}\right]}(\tilde{Z})$ is given by (3.39). The element $\left[\tau_{Z}\right]$ lies in the group

$$
\mathrm{H}_{Z}:=\left\langle\left[Z_{0}\right]^{k}\left[Z_{1}\right]\left[Z_{0}\right]^{-k}, k \in \mathbb{Z}\right\rangle
$$

As $M_{[\sigma]}(\tilde{Z})$ vanishes for $[\sigma]$ in the commutator subgroup $\mathrm{H}_{Z}^{\prime}=\left[\mathrm{H}_{Z}, \mathrm{H}_{Z}\right]$, we find

$$
M_{\left[\tau_{Z}\right]}(\tilde{Z})=\sum_{k \in \mathbb{Z}} M_{\left(\left[Z_{0}\right]^{k}\left[Z_{1}\right]\left[Z_{0}\right]^{-k}\right)^{h(k)}}(\tilde{Z}),
$$

in which $h(k)$ is the sum of exponents of $\left[Z_{0}\right]^{k}\left[Z_{1}\right]\left[Z_{0}\right]^{-k}$ occurring in $\left[\tau_{Z}\right]$. Recall that for $(s, z, c) \in \Delta$, we have, using (3.15),

$$
\begin{aligned}
M_{\left[Z_{0}\right]^{k}\left[Z_{1}\right]\left[Z_{0}\right]^{-k}}(\tilde{Z})(s, z, c) & =-\frac{(2 \pi)^{s} e^{\frac{\pi i s}{2}}}{\Gamma(s)} f_{k}(s, z, c), \\
M_{\left(\left[Z_{0}\right]^{k}\left[Z_{1}\right]\left[Z_{0}\right]^{-k}\right)^{-1}}(\tilde{Z})(s, z, c) & =-e^{-2 \pi i s} M_{\left[Z_{0}\right]^{k}\left[Z_{1}\right]\left[Z_{0}\right]^{-k}(\tilde{Z})(s, z, c),}
\end{aligned}
$$

and

$$
M_{\left(\left[Z_{0}\right]^{k}\left[Z_{1}\right]\left[Z_{0}\right]^{-k}\right)^{ \pm j}}(\tilde{Z})(s, z, c)=\frac{e^{ \pm 2 \pi i j s}-1}{e^{ \pm 2 \pi i s}-1} M_{\left(\left[Z_{0}\right]^{k}\left[Z_{1}\right]\left[Z_{0}\right]^{-k}\right)^{ \pm 1}}(\tilde{Z})(s, z, c) \text { for } k \geq(13.55)
$$

These formulas give a way to evaluate any $M_{[\tau]}(\tilde{Z})(s, z, c)$ explicitly.

Proof of Theorem 2.1. The result follows from Theorems 3.4 and 3.5.

\subsection{Extended analytic continuation}

We now obtain the extended analytic continuation of the Lerch transcendent, to $\mathcal{N}^{\#}$, the manifold obtained from $\mathcal{N}$ by gluing in the regions $\mathcal{V}(c=n):=\{(a, c): a \in \mathbb{C}, c=n\}$ for $c=n \in \mathbb{Z}_{\geq 1}$.

Theorem 3.6 (Lerch Transcendent Extended Analytic Continuation) The Lerch transcendent $\Phi(s, z, c)$ analytically continues to a single-valued function $\tilde{Z}=\tilde{Z}(s, z, c,[\gamma])$ on the universal cover $\tilde{\mathcal{N}}^{\#}$ of

$$
\mathcal{N}^{\#}=\left\{(s, z, c) \in \mathbb{C} \times\left(\mathbb{P}^{1}(\mathbb{C}) \backslash\{0,1, \infty\}\right) \times\left(\mathbb{C} \backslash \mathbb{Z}_{\leq 0}\right)\right\} .
$$

All monodromy functions $M_{[\tau]}(\tilde{Z})$ vanish identically for $[\tau]$ in the second commutator subgroup $\left(\pi_{1}\left(\mathcal{N}^{\#}, \mathbf{x}_{0}^{\prime}\right)\right)^{\prime \prime}$ of $\pi_{1}\left(\mathcal{N}^{\#}, \mathbf{x}_{0}^{\prime}\right)$. Thus the analytic continuation becomes single-valued on a covering manifold of $\mathcal{N}^{\#}$ which has a two-step solvable group of deck transformations.

Proof The fact that the possible singularities at $c=n$ are removable follows almost immediately from the corresponding result in Part II [47, Theorem 2.3]. It remains only to check that the additional monodromy functions in Theorems 3.4 and 3.5 remain holomorphic at points $(s, a, c)$ with $c=n \geq 1$, in $\tilde{\mathcal{N}}^{\#}$. This is apparent from their form given in Theorem 3.4 , i.e. the only locations where they are possibly not holomorphic are points $(s, z, c)$ with $z=0,1$ or $c \in \mathbb{Z}_{\leq 0}$. 
The manifold $\tilde{\mathcal{N}}^{\#}$ has a smaller fundamental group than $\mathcal{N}$, which is obtained as a quotient of $\pi_{1}\left(\mathcal{N}, \mathbf{x}_{0}^{\prime}\right)$ by setting all generators $\left\{\left[Y_{n}\right]: n \geq 1\right\}$ equal to the identity. However the vanishing of all relevant monodromy for $M_{\left[Y_{n}\right]}(f)$ for $n \geq 1$, for $f=\tilde{Z}$ or $f=$ $M_{[\tau]}(\tilde{Z})$ a monodromy function, allows the conclusion that all monodromy functions vanish identically for $[\tau]$ in the second commutator subgroup $\left(\pi_{1}\left(\mathcal{N}^{\#}, \mathbf{x}_{0}^{\prime}\right)\right)^{\prime \prime}$. This follows from the corresponding assertion of Theorem 3.5 for $\left(\pi_{1}\left(\mathcal{N}, \mathbf{x}_{0}^{\prime}\right)\right)^{\prime \prime}$.

Remark 3.7 The k-th lower central series group $\boldsymbol{\gamma}_{(k)}(\mathrm{G})$ of a group $\mathrm{G}$ is defined recursively by

$$
\gamma_{(k)}(\mathrm{G}):=\left[\gamma_{(k-1)}(\mathrm{G}): \mathrm{G}\right]
$$

Each quotient group $\mathrm{G} / \boldsymbol{\gamma}_{(k)}(\mathrm{G})$ is a nilpotent group. It can be shown that the group $\pi_{1}\left(\mathcal{N}, \mathbf{x}_{0}^{\prime}\right) / \Gamma^{\prime}$ is not nilpotent, where $\Gamma^{\prime}=\left[H_{0}, H_{0}\right]$ is the group given in Theorem 3.5. It follows that the lower central series group $\gamma_{(k)}\left(\pi_{1}\left(\mathcal{N}, \mathbf{x}_{0}^{\prime}\right)\right) \nsubseteq \Gamma^{\prime}$ for all $n \geq 1$. Thus the quotient group $\pi_{1}\left(\mathcal{N}, \mathbf{x}_{0}^{\prime}\right) / \Gamma^{\prime}$ falls outside the framework studied by Deligne [18].

\section{Differential-difference operators and monodromy functions}

As discussed in part II, the Lerch zeta function $\zeta(s, a, c)$ satisfies two differential-difference equations. We introduce the operators

$$
\mathrm{D}_{L}^{-}=\frac{1}{2 \pi i} \frac{\partial}{\partial a}+c \text { and } \mathrm{D}_{L}^{+}=\frac{\partial}{\partial c}
$$

Then we have

$$
\mathrm{D}_{L}^{-} \zeta(s, a, c)=\zeta(s-1, a, c)
$$

and

$$
\mathrm{D}_{L}^{+} \zeta(s, a, c)=-s \zeta(s+1, a, c)
$$

In consequence, the analytically continued Lerch zeta function satisfies the linear partial differential equation

$$
\mathrm{D}_{L} \zeta(s, a, c,[\gamma])=-s \zeta(s, a, c,[\gamma])
$$

in which

$$
\mathrm{D}_{L}:=\mathrm{D}_{L}^{-} \mathrm{D}_{L}^{+}=\frac{1}{2 \pi i} \frac{\partial}{\partial a} \frac{\partial}{\partial c}+c \frac{\partial}{\partial c} .
$$

The Lerch transcendent $\Phi(s, z, c)$ then satisfies suitable differential-difference equations and a linear partial differential equation inherited from these. The substitution $z=e^{2 \pi i a}$ transforms the Lerch differential operator to the polyzeta operator

$$
\mathrm{D}_{\Phi}:=\mathrm{D}_{\Phi}^{-} \mathrm{D}_{\Phi}^{+}=\left(z \frac{\partial}{\partial z}+c\right) \frac{\partial}{\partial c} .
$$

This lifts to an operator on functions on the universal cover $\tilde{\mathcal{N}} \equiv \tilde{\mathcal{M}}$ which is equivariant with respect to the group $\mathcal{G}$ of diffeomorphisms of $\tilde{\mathcal{N}}$ that preserve the projection from $\tilde{\mathcal{N}}$ to $\mathcal{N}$; the group $\mathcal{G}$ is isomorphic to $\pi_{1}\left(\mathcal{N}, \mathbf{x}_{0}^{\prime}\right)$. 
Theorem 4.1 (Lerch Transcendent Differential-Difference Operators)

(1) The analytic continuation $\tilde{Z}(s, z, c,[\gamma])$ of the Lerch transcendent $\Phi(s, z, c)$ on the universal cover $\tilde{\mathcal{N}}$ satisfies the two differential-difference equations

$$
\left(z \frac{\partial}{\partial z}+c\right) \tilde{Z}(s, z, c,[\gamma])=\tilde{Z}\left(s-1, z, c,\left[\gamma_{-}\right]\right),
$$

and

$$
\frac{\partial}{\partial c} \tilde{Z}(s, z, c,[\gamma])=-s \tilde{Z}\left(s+1, z, c,\left[\gamma_{+}\right]\right),
$$

in which $\left[\gamma_{+}\right]$and $\left[\gamma_{-}\right]$denote paths in $\tilde{\mathcal{N}}$ which first traverse $\gamma$ and then traverse a path from the endpoint of $\gamma$ that changes the s-variable only, moving from s to $s \pm 1$, respectively.

(2) The analytic continuation $\tilde{Z}(s, z, c,[\gamma])$ on $\tilde{\mathcal{N}}$ satisfies the linear partial differential equation

$$
\mathrm{D}_{\Phi}(\tilde{Z})(s, z, c,[\gamma])=-s \tilde{Z}(s, z, c,[\gamma])
$$

where $\mathrm{D}_{\Phi}=z \frac{\partial}{\partial z} \frac{\partial}{\partial c}+c \frac{\partial}{\partial c}$

(3) Foreach $[\tau] \in \pi_{1}\left(\mathcal{N}, \mathbf{x}_{0}^{\prime}\right)$, the Lerch transcendent monodromy function $M_{[\tau]}(\tilde{Z})(s, z, c,[\gamma])$ satisfies on $\tilde{\mathcal{N}}$ the two differential-difference equations and the linear differential equation above.

Proof (1), (2) These results for $\tilde{Z}(s, z, c,[\gamma])$ follow directly from Theorem 5.1 of part II by a change of variable $z=e^{2 \pi i a}$. Here we have

$$
z \frac{\partial}{\partial z}=\frac{1}{2 \pi i} \frac{\partial}{\partial a} .
$$

Thus in the $(s, z, c)$-variables, the corresponding differential-difference equations satisfied by the Lerch transcendent are

$$
\left(z \frac{\partial}{\partial z}+c\right) \Phi(s, z, c)=\Phi(s-1, z, c)
$$

and

$$
\frac{\partial}{\partial c} \Phi(s, z, c)=-s \Phi(s+1, z, c)
$$

and the corresponding differential equation lifts to that given in (4.9) by analytic continuation.

(3) The monodromy functions satisfy the same differential-difference equations and differential equation because the differential operators $z \frac{\partial}{\partial z}$ and $\frac{\partial}{\partial c}$ are equivariant with respect to the covering map from $\tilde{\mathcal{N}}$ to $\mathcal{N}$.

We now study the restricted Lerch transcendent monodromy functions $M_{\left[\tau^{\prime}\right]}^{s}(\tilde{Z})$ obtained by holding the variable $s$ fixed, in a fashion analogous to $\$ 7$ of part II. 
Definition 4.2 The Lerch transcendent monodromy space $\mathcal{W}_{s}$ is the $\mathbb{C}$-vector space spanned by all functions $\mathrm{Q}_{\left[\tau^{\prime}\right]}^{s}(\tilde{Z})$ (as $\left[\tau^{\prime}\right]$ varies) regarded as functions on the (simply connected) submanifold

$$
\tilde{\mathcal{N}}_{s}:=(\pi)^{-1}\left(\{s\} \times\left(\mathbb{P}^{1}(\mathbb{C}) \backslash\{0,1, \infty\}\right)\right) \times(\mathbb{C} \backslash \mathbb{Z}) \subset \tilde{\mathcal{N}}
$$

where $\pi: \tilde{\mathcal{N}} \rightarrow \mathcal{N}$ is the universal covering map. Here $\tilde{Z}^{s}:=\mathrm{Q}_{[i d]}(\tilde{Z})$ is the restriction of $\tilde{Z}$ to $\tilde{\mathcal{N}}_{s}$. This vector space is a direct sum of one-dimensional spaces:

$$
\mathcal{W}_{s}:=\mathbb{C}\left[\tilde{Z}^{s}\right] \bigoplus\left(\sum_{\left[\tau^{\prime}\right] \in \pi_{1}\left(\mathcal{N}, \mathbf{x}_{0}^{\prime}\right)} \mathbb{C}\left[M_{\left[\tau^{\prime}\right]}^{s}(\tilde{Z})\right]\right)
$$

consisting of all finite linear combinations of the given countable generating set of vectors.

These vector spaces have the following properties:

Theorem 4.3 (Lerch Transcendent Monodromy Space) The Lerch transcendent monodromy space $\mathcal{W}_{s}$ at $s$ depends on the parameter $s \in \mathbb{C}$ as follows.

(i) (Generic Case) If $s \notin \mathbb{Z}$, then $\mathcal{W}_{s}$ is an infinite-dimensional vector space and has as a basis the set of functions

$$
\left\{M_{\left[Z_{0}\right]^{k}\left[Z_{1}\right]\left[Z_{0}\right]^{-k}}^{s}(\tilde{Z}): k \in \mathbb{Z}\right\} \cup\left\{M_{\left[Y_{n}\right]}^{s}(\tilde{Z}): n \in \mathbb{Z}\right\} \cup\left\{\tilde{Z}^{s}\right\}
$$

(ii) (Positive Integer Case) If $s=m \in \mathbb{Z}_{>0}$, then $\mathcal{W}_{m}$ is an infinite-dimensional vector space and has as a basis the set of functions

$$
\left\{M_{\left[Z_{0}\right]^{k}\left[Z_{1}\right]\left[Z_{0}\right]^{-k}}^{m}(\tilde{Z}): k \in \mathbb{Z}\right\} \cup\left\{\tilde{Z}^{m}\right\}
$$

(iii) (Nonpositive Integer Case) If $s=-m \in \mathbb{Z}_{\leq 0}$, then all Lerch transcendent monodromy functions vanish identically, i.e.

$$
M_{[\tau]}^{-m}(\tilde{Z})=0 \quad \text { for all }[\tau] \in \pi_{1}\left(\mathcal{N}, \mathbf{x}_{0}^{\prime}\right)
$$

Thus $\mathcal{W}_{-m}=\mathbb{C}\left[\tilde{Z}^{-m}\right]$ is a one-dimensional vector space.

Proof We establish these cases in reverse order:

(iii) Theorem 3.4 shows that for $s=-m \in \mathbb{Z}_{\leq 0}$ the monodromy functions of all generators $[S] \in \mathcal{G}^{\prime}$ and their inverses vanish identically. In the case of $M_{\left[Z_{1}\right]}^{-m}(\tilde{Z})$ this is because $\frac{1}{\Gamma(-m)}=0$. This carries over to all $\left[\tau^{\prime}\right] \in \pi_{1}\left(\mathcal{N}, \mathbf{x}_{0}^{\prime}\right)$ by induction in the length of a word expressing $\left[\tau^{\prime}\right]$ in terms of the generators. Only the original function $\tilde{Z}$ remains.

(ii) For $s=m \in \mathbb{Z}_{\geq 1}$, the monodromy of all $\left\{M_{\left[Y_{n}\right]}^{m}(\tilde{Z}): n \in \mathbb{Z}\right\}$ vanishes identically. Theorem 3.5 implies that the space $\mathcal{W}_{m}$ is spanned by $\tilde{Z}^{s}=\mathrm{Q}_{[i d]}(\tilde{Z})$ together with $\left\{M_{\left[Z_{0}\right]^{k}\left[Z_{1}\right]\left[Z_{0}\right]^{-k}}^{m}(\tilde{Z}): n \in \mathbb{Z}\right\} \cup\left\{\tilde{Z}^{m}\right\}$. The formulas in Theorem 3.4 (i) then indicate that each $M_{\left[Z_{0}\right]^{k}\left[Z_{1}\right]\left[Z_{0}\right]^{-k}}^{m}(\tilde{Z})$ is a nonzero multiple of $\left.f_{n}\right|_{s=m}$, where $f_{n}$ at $(s, z, c) \in \Delta$ is given by (3.24). Thus

$$
\mathcal{W}_{m}:=\mathbb{C}\left[\tilde{Z}^{s}\right] \bigoplus\left(\oplus_{n \in \mathbb{Z}} \mathbb{C}\left[\left.f_{n}\right|_{s=m}\right] .\right)
$$


Each finite subset of the functions $\left.f_{n}(s, z, c)\right|_{s=m}$ together with $\tilde{Z}^{s}$ is easily checked to be linearly independent in a small neighborhood of the point $\left(m,-1, \frac{1}{2}\right)$, so (ii) follows.

(i) The proof in the generic case parallels that of Theorem 7.1 of part II ([48]). It also makes use of the independence formula (3.38) of Theorem 3.5.

Remark 4.4 Theorem 4.3 shows that values $s \in \mathbb{Z}$ are "special values" in the sense that the monodromy functions satisfy non-generic linear relations at these values. We note the coincidence that these same points $s \in \mathbb{Z}$ are "special values" in the sense of number theory, in that the values of these functions at these points encode important arithmetic information, discussed below. Non-positive integer $s=-m \leq 0$ are especially interesting because all monodromy functions vanish identically: therefore, the values at these points are well-defined on the base manifold $\mathcal{N}$, without having to lift to any covering manifold. This observation strengthens the observation made in part II of the vanishing property of monodromy for the Lerch zeta function, because we have a larger set of monodromy functions. The "well-definedness" property of these values seems particularly significant in that these values contain arithmetic information: $p$-adic $L$-functions can be obtained by $p$-adic interpolation through values at these points. More precisely, results in $\$ 6$ establish the following:

(i) The values of the periodic zeta function $F(a, s)=\sum_{n=1}^{\infty} \frac{e^{2 \pi i n a}}{n^{s}}$ at negative integers $s=$ $-m \leq 0$, corresponding to a singular strata degenerations of the Lerch transcendent, are recoverable as limits of non-singular Lerch transcendent values in this paper.

(ii) The classical $p$-adic $L$-functions can be constructed by $p$-adic interpolation starting from values of $F(a,-m)$.

Remark 4.5 Theorem 4.3 treats the monodromy functions as multivalued functions of two variables $(z, c)$. If the variable $c$ is also fixed, so that the monodomy functions depend on only the variable $z$, then in Sect. 8 we show that the monodromy vector spaces become finite-dimensional when $s=m \in \mathbb{Z}_{>0}$ is a positive integer, see Theorem 8.1.

\section{Specialization of Lerch transcendent: $\boldsymbol{s}$ a non-positive integer}

In this and the next section we specialize $s=-m(m \geq 0)$ to be a nonpositive integer. In Theorem 2.4 (3) we observed that the values $s=-m$ are distinguished by the fact that all monodromy functions vanish identically as functions of $(z, c)$. At these values of $s$ a great simplification occurs and the resulting two-variable functions are rational functions of $(z, c)$. These rational functions of two variables were determined by Apostol [2, Section $3]$ in 1951. They were later studied by Kanemitsu, Katsurada, and Yoshimoto [38, Sect. 4], who obtained various formulas for them, cf. their Theorem 6 .

Specifically, for $z=-m(M \geq 0)$ the modified function

$$
L i_{-m}(z, c):=z \Phi(-m, z, c)=\sum_{n=0}^{\infty}(n+c)^{m} z^{n+1},
$$

is a $c$-deformed polylogarithm of negative integer order. As mentioned above, it is known that the functions $L i_{-m}(z, c)$ meromorphically continue as rational functions of two variables $(z, c) \in \mathbb{C} \times \mathbb{C}$. These rational functions automatically give a meromorphic continuation in the $(z, c)$-variables to all integer points $c \in \mathbb{Z}$, thus including the singular strata points $c=-n \leq 0$ outside the analytic continuation of part II. 
In this section we determine recursions for these rational functions and deduce various symmetry properties they exhibit (Theorem 5.3). We begin with the following expression for $c$-deformed negative polylogarithms $L i_{-m}(z, c)$ :

Theorem 5.1 (c-Deformed Negative Polylogarithms) For $s=-m \in \mathbb{Z}_{\leq 0}$ the function $L i_{-m}(z, c)$ analytically continues to a rational function of $z$ and $c$ on $\mathbb{P}^{1}(\mathbb{C}) \times \mathbb{P}^{1}(\mathbb{C})$. Here $\operatorname{Li}_{0}(z, c)=z q_{0}(z)$ and

$$
L i_{-m}(z, c)=z\left(\sum_{k=0}^{m}\left(\begin{array}{l}
m \\
k
\end{array}\right) c^{k} q_{m-k}(z)\right), \quad \text { for } m \geq 1,
$$

in which the $q_{m}(z)$ are rational functions given by

$$
q_{0}(z)=\frac{1}{1-z}
$$

and

$$
q_{m+1}(z)=z \frac{d}{\mathrm{~d} z}\left(q_{m}(z)\right) \quad \text { for } \quad m \geq 0 .
$$

Proof The case $m=0$ with $c \neq 0, \infty$ follows immediately from (1.9),

$$
L i_{0}(z, c)=z\left(\sum_{n=0}^{\infty} z^{n}\right)=\frac{z}{1-z} .
$$

Since this function is independent of $c$, it extends trivially to all $c \in \mathbb{P}^{1}(\mathbb{C})$ as $L i_{0}(z, c)$. We next use the identity

$$
\Phi(s-1, z, c)=\left(z \frac{\partial}{\partial z}+c\right) \Phi(s, z, c)
$$

which yields, taking $s=-m$,

$$
L i_{-m-1}(z, c)=z\left(z \frac{\partial}{\partial z}+c\right)\left(z^{-1} L i_{-m}(z, c)\right) .
$$

This holds for $c \neq 0, \infty$ when $z$ is in a sufficiently small circular neighborhood of the origin (with radius depending on both $c$ and $m$ ). The fact that the function $L i_{-m}(z, c)$ has the form (5.2) for all $m \geq 1$ with $q_{j}(z)$ as described is proved by induction on $m \geq 1$ using (5.5), taking the differential Eq. (5.4) as the definition of $q_{m+1}(z)$. The differential Eq. (5.4) shows that each $q_{m}(z)$ is a rational function of $z$.

In the remainder of this section we study these "special values" with $s=-m \in \mathbb{Z}_{\leq 0}$ in more detail. One easily shows by induction on $m$ that the rational function $q_{m}(z)$ has the form

$$
q_{m}(z)=\frac{r_{m}(z)}{(1-z)^{m+1}}
$$

in which $r_{m}(z) \in \mathbb{Z}[z]$ is a monic polynomial of degree $m$. For $m \geq 1$ we have $r_{m}(0)=0$ and $r_{m}(1)=m$ !. The first few values of $r_{m}(z)$ are given in the following Table 1 .

To evaluate the rational functions $q_{m}(z)$ we use the following result, which is due to Apostol [2, (3.1)]. 
Table 1 Values of $r_{m}(z)$

\begin{tabular}{ll}
\hline $\mathbf{m}$ & $\boldsymbol{r}_{\boldsymbol{m}}(\boldsymbol{z})$ \\
\hline 1 & $z$ \\
2 & $z^{2}+z$ \\
3 & $z^{3}+4 z^{2}+z$ \\
4 & $z^{4}+11 z^{3}+11 z^{2}+z$ \\
5 & $z^{5}+26 z^{4}+66 z^{3}+26 z^{2}+z$ \\
\hline
\end{tabular}

Lemma 5.2 Let

$$
G(z, c ; u):=\sum_{m=0}^{\infty} L i_{-m}(z, c) \frac{u^{m}}{m !}
$$

be the exponential generating function for the functions $\left\{L i_{-m}(c, z): m \geq 0\right\}$. This series converges absolutely on the region $\left\{(z, c, u):|z|<e^{-|u|}, c \in \mathbb{C}\right\}$ where it is given by

$$
G(z, c ; u)=\frac{z e^{c u}}{1-z e^{u}} .
$$

The right side of (5.8) gives a meromorphic continuation of $G(z, c ; u)$ to $(z, c, u) \in \mathbb{C}^{3}$.

Proof For $|z|<1$ we have

$$
L i_{-m}(z, c)=\sum_{k=0}^{\infty} z^{k+1}(k+c)^{m},
$$

where the series converges absolutely for all $c \in \mathbb{C}$. If in addition $|z|<e^{-|u|}$, then we have the bounds

$$
\begin{aligned}
\sum_{m=0}^{\infty} \sum_{k=0}^{\infty}|z|^{k+1}(k+|c|)^{m} \frac{|u|^{m}}{m !} & =\sum_{m=0}^{\infty} \sum_{k=0}^{\infty}|z|^{k+1} \frac{((k+|c|)|u|)^{m}}{m !} \\
& \leq \sum_{k=0}^{\infty}|z|^{k+1} e^{(k+|c|)|u|}=e^{|c u|-|u|}\left(\frac{1}{1-|z| e^{|u|}}\right)<\infty .
\end{aligned}
$$

Thus when $|z|<e^{-|u|}$, we have

$$
\begin{aligned}
\sum_{m=0}^{\infty} L i_{-m}(z, c) \frac{u^{m}}{m !} & =\sum_{m=0}^{\infty}\left(\sum_{k=0}^{\infty} z^{k+1}(k+c)^{m}\right) \frac{u^{m}}{m !} \\
& =\sum_{k=0}^{\infty} z^{k+1}\left(\sum_{m=0}^{\infty} \frac{((k+c) u)^{m}}{m !}\right) \\
& =\sum_{k=0}^{\infty} z^{k+1} e^{(k+c) u}=\frac{z e^{c u}}{1-z e^{u}},
\end{aligned}
$$

which is (5.8).

We now establish various properties of the rational functions $q_{m}(z)$ appearing in these special values.

Theorem 5.3 ( Properties of $q_{m}(z)$ ) For $m \geq 0$ the rational functions $q_{m}(z)=\frac{r_{m}(z)}{(1-z)^{m+1}}$, where $r_{m}(z)$ is a monic polynomial of degree exactly $m$, having $r_{m}(1)=m !$. These polynomials have the following properties: 
(i) (Laurent expansion). The Laurent expansion $q_{m}(z)=\sum_{k=0}^{m+1} \frac{a_{m, k}}{(1-z)^{k}}$ around $z=1$ is given by

$$
r_{m}(z)=\sum_{k=0}^{m+1} a_{m, k}(1-z)^{m+1-k}
$$

in which $a_{m, 0}=0$ for $m \geq 0$, and for $1 \leq k \leq m+1$ we have

$$
a_{m, k}=(-1)^{m} \sum_{l=0}^{k-1}(-1)^{l}\left(\begin{array}{c}
k-1 \\
l
\end{array}\right)(l+1)^{m}
$$

(ii) (Reflection symmetry). For $m \geq 1$,

$$
z^{m+1} r_{m}\left(\frac{1}{z}\right)=r_{m}(z)
$$

(iii) (Recursion). The polynomials $r_{m}(z)$ satisfy

$$
r_{m}(z)=z \sum_{j=1}^{m}\left(\begin{array}{c}
m \\
j
\end{array}\right) r_{m-j}(z)(1-z)^{j-1}
$$

Proof We specialize the $c$ variable to $c=0$ in Theorem 5.1 to obtain

$$
L i_{-m}(z, 0)=z q_{m}(z)
$$

Letting $c=0$ in Lemma 5.2 and dividing by $z$ then yields the exponential generating function

$$
\sum_{m=0}^{\infty} q_{m}(z) \frac{u^{m}}{m !}=\frac{1}{1-z e^{u}}
$$

(i) Theorem 5.1 implies that the rational function $q_{m}(z)$ has the form $\sum_{k=0}^{m+1} \frac{a_{m, k}}{(1-z)^{k}}$. Substituting this expression for $q_{m}(z)$ into the left-hand side of (5.14) and interchanging the order of summation over $m$ and $k$ yields

$$
\sum_{m=0}^{\infty} q_{m}(z) \frac{u^{m}}{m !}=\sum_{m=0}^{\infty} \sum_{k=0}^{m+1} \frac{a_{m, k}}{(1-z)^{k}} \frac{u^{m}}{m !}=\sum_{k=1}^{\infty} \frac{1}{(1-z)^{k}}\left(\sum_{m=k-1}^{\infty} a_{m, k} \frac{u^{m}}{m !}\right) .
$$

We express the right-hand side of (5.14) also as an infinite series in powers of $\frac{1}{1-z}$ and $u$, valid for $\left|1-e^{-u}\right|<|1-z|$ :

$$
\begin{aligned}
\frac{1}{1-z e^{u}} & =\frac{e^{-u}}{e^{-u}-z}=\frac{e^{-u}}{(1-z)-\left(1-e^{-u}\right)}=\frac{1}{1-z} \cdot \frac{e^{-u}}{1-\frac{1-e^{-u}}{1-z}} \\
& =\frac{1}{1-z} \cdot e^{-u} \sum_{l=0}^{\infty}\left(\frac{1-e^{-u}}{1-z}\right)^{l}=\sum_{k=1}^{\infty} \frac{1}{(1-z)^{k}} \cdot e^{-u}\left(1-e^{-u}\right)^{k-1}
\end{aligned}
$$




$$
\begin{aligned}
& =\sum_{k=1}^{\infty} \frac{1}{(1-z)^{k}}\left(\sum_{l=0}^{k-1}(-1)^{l}\left(\begin{array}{c}
k-1 \\
l
\end{array}\right) e^{-(l+1) u}\right) \\
& =\sum_{k=1}^{\infty} \frac{1}{(1-z)^{k}}\left(\sum_{l=0}^{k-1}(-1)^{l}\left(\begin{array}{c}
k-1 \\
l
\end{array}\right)\left(\sum_{m=0}^{\infty}(-1)^{m}(l+1)^{m} \frac{u^{m}}{m !}\right)\right) .
\end{aligned}
$$

Comparing the coefficients of $\frac{1}{(1-z)^{k}} \frac{u^{m}}{m !}$ in (5.15) and (5.16) gives the expression for $a_{m, k}$.

(ii) The proof of this identity also uses (5.14). More precisely, replacing $z$ by $\frac{1}{z}$ in (5.14), we obtain

$$
\sum_{m=0}^{\infty} q_{m}\left(\frac{1}{z}\right) \frac{u^{m}}{m !}=\frac{1}{1-\frac{1}{z} e^{u}}=\frac{z e^{-u}}{z e^{-u}-1}=1-\frac{1}{1-z e^{-u}}=1-\sum_{m=0}^{\infty} q_{m}(z) \frac{(-u)^{m}}{m !} .
$$

This gives the relation

$$
q_{m}\left(\frac{1}{z}\right)=(-1)^{m+1} q_{m}(z) \text { for } m \geq 1 .
$$

Since

$$
q_{m}(z)=\frac{r_{m}(z)}{(1-z)^{m+1}}
$$

the above identity can be rewritten as

$$
\frac{z^{m+1} r_{m}\left(\frac{1}{z}\right)}{(z-1)^{m+1}}=\frac{r_{m}\left(\frac{1}{z}\right)}{\left(1-\frac{1}{z}\right)^{m+1}}=(-1)^{m+1} \frac{r_{m}(z)}{(1-z)^{m+1}}
$$

which proves (ii).

(iii) Dividing both sides of (5.12) by $(1-z)^{m+1}$, we convert (5.12) to an equivalent form

$$
q_{m}(z)=\frac{z}{1-z} \sum_{j=1}^{m}\left(\begin{array}{c}
m \\
j
\end{array}\right) q_{m-j}(z) \text { for } m \geq 1 .
$$

When $m=1$, the right-hand side is $\frac{z}{1-z} q_{0}(z)=\frac{z}{(1-z)^{2}}=q_{1}(z)$. We shall prove (5.17) by induction on $m$. Assume it holds for some $m \geq 1$. Rewrite the identity in this case as

$$
(1-z) q_{m}(z)=z \sum_{j=1}^{m}\left(\begin{array}{c}
m \\
j
\end{array}\right) q_{m-j}(z)
$$

Differentiating both sides of (5.18) and using the identity (5.4) that $q_{n+1}(z)=$ $z \frac{\partial}{\partial z}\left(q_{n}(z)\right)$ for $n \geq 0$, we arrive at

$$
\begin{aligned}
-q_{m}(z)+(1-z) \frac{\partial}{\partial z}\left(q_{m}(z)\right) & =\sum_{j=1}^{m}\left(\begin{array}{c}
m \\
j
\end{array}\right) q_{m-j}(z)+z \sum_{j=1}^{m}\left(\begin{array}{c}
m \\
j
\end{array}\right) \frac{\partial}{\partial z} q_{m-j}(z) \\
& =\sum_{j=1}^{m}\left(\begin{array}{c}
m \\
j
\end{array}\right) q_{m-j}(z)+\sum_{j=1}^{m}\left(\begin{array}{c}
m \\
j
\end{array}\right) q_{m-j+1}(z) \\
& =q_{0}(z)+\sum_{j=2}^{m}\left(\begin{array}{c}
m+1 \\
j
\end{array}\right) q_{m-j+1}(z)+m q_{m}(z) .
\end{aligned}
$$


In other words,

$$
(1-z) \frac{\partial}{\partial z}\left(q_{m}(z)\right)=\sum_{j=1}^{m+1}\left(\begin{array}{c}
m+1 \\
j
\end{array}\right) q_{m+1-j}(z) .
$$

Therefore,

$$
q_{m+1}(z)=z \frac{\partial}{\partial z}\left(q_{m}(z)\right)=\frac{z}{1-z} \sum_{j=1}^{m+1}\left(\begin{array}{c}
m+1 \\
j
\end{array}\right),
$$

as desired.

\section{Double specialization: periodic zeta function}

We now consider the double specialization of the Lerch transcendent setting $s=-m$ a non-positive integer and setting $c=0$. The importance of these "special values" $s=-m$ of the $s$-parameter is that values of zeta and $L$-functions at these points have arithmetic significance; they encode information about the arithmetic structure of number fields. We have the problem that the $c$-parameter value $c=0$ lies on the singular stratum outside the analytic continuation of $\Phi(s, z, c)$ given in Sect. 3. Our object is to show that sufficiently many of these "special values" can be recovered by a limiting process from "regular stratum" values of the Lerch transcendent so as to carry out the number-theoretic construction of $p$-adic $L$-functions. We shall take a limit as $c \rightarrow 0$, and to do this we set $z=e^{2 \pi i a}$ and will also suppose that $0<\Re(a)<1$, so that $z \in \mathbb{C} \backslash \mathbb{R}_{>0}$.

Concerning the number theoretic significance of the values $s=-m(m \geq 0)$ is that on a singular stratum of the Lerch transcendent giving the Riemann zeta function these values are the important rational numbers

$$
\zeta(-k)=-\frac{B_{k+1}}{k+1},
$$

where the $B_{k}$ are Bernoulli numbers. We follow the convention on Bernoulli numbers that they are defined by

$$
\frac{t}{e^{t}-1}=\sum_{k=1}^{\infty} \frac{B_{k}}{k !} t^{k}
$$

More generally the special values $L(-m, \chi)$ of Dirichlet $L$-functions, for $m \geq 0$ are arithmetically important algebraic numbers. These values can be expressed as linear combinations of either Hurwitz zeta function values $\zeta\left(-m, \frac{a}{d}\right)=\Phi\left(-m, 1, \frac{a}{d}\right)$ or, alternatively, of periodic zeta function values $P\left(\frac{a}{d},-m\right)=\Phi\left(-m, e^{2 \pi i \frac{a}{d}}, 1\right)$ taken at values which are roots of unity in the $z$ variable.

The arithmetic information in the "special values" $L(-m, \chi)$ include $p$-adic regularities captured by interpolating these values $p$-adically to obtain $p$-adic $L$-functions. Two different interpolation methods to construct $p$-adic $L$-functions are known. A original construction of Kubota and Leopoldt [43] in 1964 used interpolation of Hurwitz zeta function values; it is presented in Washington [73, Sect. 5.2,Theorem 5.11]. A second approach was given in 1977 by Morita [56]. which uses interpolation of periodic zeta function values, at the points $(z, a)$ with $a=\frac{j}{p^{k}}$ having $0<j<p^{k}$ and with $z=e^{\frac{2 \pi i j}{p^{k}}}$. More information on the periodic zeta function approach to $p$-adic $L$-functions is given in Amice and Fresnel [1] and Naito [59]. 
We use the periodic zeta function approach to the "special values", where limits exist, rather than the Hurwitz zeta function approach, where the limits do not exist, see Remark 6.2 below. The following result shows that it is possible to take a limit as $c \rightarrow 0^{+}$of $z \Phi(s, z, c)$, with $z=e^{2 \pi i a}$ to recover the values at $s=-m$ of the (analytically continued) periodic zeta function

$$
F(a, s)=\sum_{n=1}^{\infty} \frac{e^{2 \pi i n a}}{n^{s}} .
$$

That is, this limit exists, and these values $F(z, s)$ are extractable from data in the nonsingular part of the analytic continuation of $\Phi(s, z, c)$ at $z=-m$, when approaching the singularity.

Theorem 6.1 (Periodic Zeta Function Special Values)

(1) For $z \in \mathbb{P}^{1}(\mathbb{C}) \backslash\{0,1, \infty\}$ and $-m \in \mathbb{Z}_{\leq 0}$ there holds

$$
L i_{-m}(z, 0)=\lim _{c \rightarrow 0^{+}} L i_{-m}(z, c)=\lim _{c \rightarrow 0^{+}} z \Phi(-m, z, c)
$$

where the limit is taken through values of $c$ in $0<\Re(c)<1$.

(2) For $0<\Re(a)<1$ the periodic zeta function $F(a, s)=\sum_{n=1}^{\infty} \frac{e^{2 \pi i n a}}{n^{s}}$ analytically continues to an entire function of $s$. In particular, for $s=-m \in \mathbb{Z}_{\leq 0}$ there holds

$$
F(a,-m)=e^{-2 \pi i a} L i_{-m}\left(e^{2 \pi i a}, 0\right)=q_{m}\left(e^{2 \pi i a}\right)
$$

Proof (1) By Theorem $5.1 L i_{-m}(z, c)$ is a rational function of $(z, c)$, giving the left equality in

$$
L i_{-m}(z, 0)=\lim _{c \rightarrow 0^{+}} L i_{-m}(z, c)
$$

where the limit is taken over values in $0<\Re(c)<1$. Now the equality $\operatorname{Li}_{-m}(z, c)=$ $z \Phi(-m, z, c)$ (which holds by analytic continuation) gives the result (6.1).

(2) The analytic continuation of $F(a, s)$ to an entire function of $s$ for $0<\mathfrak{R}(a)<1$ follows directly from the integral representation

$$
F(a, s)=\frac{1}{\Gamma(s)} \int_{0}^{\infty} \frac{1}{1-e^{2 \pi i a} e^{-t}} t^{s-1} \mathrm{~d} t,
$$

since $e^{2 \pi i a}$ stays off the nonnegative real axis.

In (3.20) we have shown that on the extended fundamental polycylinder

$$
\tilde{\Omega}=\{s \in \mathbb{C}\} \times\{a: 0<\mathfrak{R}(a)<1\} \times\{c: 0<\Re(c)<1\}
$$

we have

$$
\Phi\left(s, e^{2 \pi i a}, c\right)=\zeta(s, a, c)
$$

We now suppose $a$ is real and apply the results from part I ([46]) on the limiting behavior of the Lerch zeta function $\zeta(s, a, c)$ approaching the boundary of $\square^{\circ}$. Namely, for $\Re(s)<0$, Theorem 2.3 (iii) and equation (2.13) of [46] give for $0<a<1$ that

$$
\lim _{c \rightarrow 0^{+}} \zeta_{*}(s, a, c)=\zeta_{*}(s, a, 0)=F(a, s)
$$


in which $F(a, s)$ is the analytic continuation to $s \in \mathbb{C}$ of the periodic zeta function in the $s$-variable. Here $\zeta_{*}(s, a, c)$ is the analytic continuation in the $s$-variable of

$$
\zeta_{*}(s, a, c)=\sum_{n+c>0} e^{2 \pi i n a}(n+c)^{-s},
$$

and it satisfies $\zeta_{*}(s, a, c)=\zeta(s, a, c)$ for $(a, c) \in \square^{\circ}$. A key point is that the $n=0$ term $c^{-s}$ approaches 0 as $c \rightarrow 0^{+}$, when $\Re(s)<0$.

Now (6.3)-(6.5) give, taking $z=e^{2 \pi i a}$ with $0<a<1$ and $s=-m$ that

$$
\begin{aligned}
F(-m, s) & =\lim _{c \rightarrow 0^{+}} \zeta(-m, a, c) \\
& =\lim _{c \rightarrow 0^{+}} \Phi\left(-m, e^{2 \pi i a}, c\right) \\
& =e^{-2 \pi i a} L i_{-m}\left(e^{e \pi i a}, 0\right),
\end{aligned}
$$

where the limits are taken over real $0<c<1$. Finally, the validity of (6.2) extends from real $a$ to $0<\Re(a)<1$ by uniqueness of analytic continuation in the $a$-variable.

Remark 6.2 The Kubota-Leopoldt [43] construction of $p$-adic $L$-functions in 1964 used interpolation of special values of the Hurwitz zeta function. However, it is not possible to obtain Hurwitz zeta function special values directly by a limiting process involving the Lerch transcendent. Theorem 2.3 of Part I shows that the Hurwitz zeta function values $\zeta(s, c)$ at negative integers $s=-m$ cannot be obtained as a limit of values $\Phi(s, z, c)$, as the parameter $a=\frac{1}{2 \pi i} \log z$ has $a \rightarrow 0$ (resp. $a \rightarrow 1$ ). Under a variable change this limit corresponds to taking $z \rightarrow 1$, which is a singular stratum value. As indicated by Theorem 6.1 above we can indirectly access Hurwitz zeta function values by expressing them as a linear combination of periodic zeta function values, see Apostol [3, Theorem 12.6]. The periodic zeta function values are obtainable as limiting values using Theorem 6.1.

\section{Specialization of Lerch transcendent: $s$ a positive integer}

In this section we treat specialization of variables related to polylogarithms, where we specialize the $s$-parameter to be a positive integer value $s=m \geq 1$. We will state results in terms of the extended polylogarithm

$$
L i_{m}(z, c):=z \Phi(m, z, c)=\sum_{k=0}^{\infty} \frac{z^{k+1}}{(k+c)^{m}}
$$

Recall that the classical polylogarithm $L i_{m}(z)$ is defined by

$$
L i_{m}(z):=\sum_{k=1}^{\infty} \frac{z^{k}}{k^{m}},
$$

for integer $m \geq 1$, see Lewin $[52,53]$. The function $\operatorname{Li}_{m}(z, c)$ is related to the classical polylogarithm by taking $c=1$, with

$$
L i_{m}(z)=L i_{m}(z, 1) \text { for } m \geq 1 \text {. }
$$

This function is included in the analytic continuation of the Lerch transcendent in Theorem 3.6. 
First note that for fixed $s \in \mathbb{C}$ as a function of two variables $F(z, c):=L i_{s}(z, c)$ satisfies the linear PDE

$$
\left(z\left(z \frac{\partial}{\partial z}+c\right) \frac{1}{z}\right) \frac{\partial}{\partial c} L i_{s}(z, c)=-s L i_{s}(z, c),
$$

which easily follows from the linear PDE (1.7) satisfied by $\Phi(s, z, c)$. Using the identity

$$
z\left(z \frac{\partial}{\partial z}+c\right) \frac{1}{z}=z \frac{\partial}{\partial z}+c-1
$$

and specializing to $s=m \in \mathbb{Z}$, we obtain

$$
\left(z \frac{\partial}{\partial z} \frac{\partial}{\partial c}+c \frac{\partial}{\partial c}-\frac{\partial}{\partial c}+m\right) L i_{m}(z, c)=0 .
$$

We now show that for $s=m \geq 1$ there is an analytic continuation of $L i_{m}(z, c)$ in the two variables $(z, c)$ that meromorphically extends to all $c \in \mathbb{C}$, including positive and negative integer values. Note that this continuation in two variables extends to nonpositive integer values of $c$ that fall in a "singular stratum" outside the three-variable analytic continuation in Theorem 3.6.

Theorem 7.1 (c-Deformed Polylogarithm Analytic Continuation) For each integer $m \geq 1$ the function $L i_{m}(z, c)$ has a meromorphic continuation in two variables $(z, c)$ to the universal cover $\tilde{\mathbb{C}}_{0,1, \infty} \times \mathbb{C}$ of $\left(\mathbb{P}^{1}(\mathbb{C}) \backslash\{0,1, \infty\}\right) \times \mathbb{C}$. For fixed $\tilde{z} \in \tilde{\mathbb{C}}_{01 \infty}$, this function is meromorphic as a function of $c \in \mathbb{C}$, with its singularities consisting of poles of exact order $m$ at each of the points $c \in \mathbb{Z}_{\leq 0}$.

Proof As shown in the proof of Theorem 4.1 of part II, the Lerch zeta function $\zeta(s, a, c)$ extends to a single-valued holomorphic function on any simply connected domains contained in

$$
\mathcal{O}_{1}^{\infty}:=\{s: \Re(s)>0\} \times\{a: a \in \mathbb{C} \backslash \mathbb{Z}\} \times\{c: \Re(c)>0\}
$$

Since the monodromy functions over $\mathcal{O}_{1}^{\infty}$ are holomorphic in $c$, for each $m \in \mathbb{Z}_{>0}$, the function $L i_{m}(z, c)$ defined for $|z|<1$ and $\Re(c)>0$ by

$$
L i_{m}(z, c)=\sum_{k=0}^{\infty} \frac{z^{k+1}}{(k+c)^{m}}
$$

extends to a holomorphic function on the covering space $\tilde{\mathbb{C}}_{0,1, \infty} \times\{c: \Re(c)>0\}$. To determine its behavior on the region $\mathfrak{R}(c)>-N$, rewrite (7.7) as

$$
\begin{aligned}
L i_{m}(z, c) & =\frac{z}{c^{m}}+\frac{z^{2}}{(c+1)^{m}}+\cdots+\frac{z^{N}}{(c+N-1)^{m}}+\sum_{k=N}^{\infty} \frac{z^{k+1}}{(k+c)^{m}} \\
& =\frac{z}{c^{m}}+\frac{z^{2}}{(c+1)^{m}}+\cdots+\frac{z^{N}}{(c+N-1)^{m}}+z^{N} L i_{m}(z, c+N) .
\end{aligned}
$$

The analytic continuation of $L i_{m}(z, c)$ to $\tilde{\mathbb{C}}_{0,1, \infty} \times\{c: \Re(c)>-N\}$ is given by the right side of (7.8), which clearly shows that for a fixed $\tilde{z} \in \tilde{\mathbb{C}}_{0,1, \infty}$, the function $L i_{m}(\tilde{z}, c)$ as a function of $c$ has poles of order $m$ at $c=0,-1, \ldots,-N+1$. Theorem 7.1 follows on letting $N \rightarrow \infty$. 


\section{Double specialization: Deformed polylogarithm}

We next study the extended polylogarithm $L i_{m}(z, c)$ when two variables $s=m \in \mathbb{Z}_{\geq 1}$ and $c \in \mathbb{C}$ are fixed, and only the parameter $z$ varies. This is a double specialization. We view the $c$-parameter as giving a deformation of the polylogarithm, and to emphasize this we rewrite it as

$$
L i_{m, c}(z):=L i_{m}(c, z)=z \Phi(m, z, c) .
$$

We observe that this function of $z$ satisfies a linear ordinary differential equation, of order $m+1$ with rational function coefficients in $\mathbb{C}(z)$. We show that for all $c \in \mathbb{C}$, its singular points are at $\{0,1, \infty\}$ and that is of Fuchsian type. Then we determine its monodromy as a function of the parameter $c$. We observe that the monodromy representation varies continuously in the parameter $c$ at nonsingular values $c \in \mathbb{C} \backslash \mathbb{Z}_{\leq 0}$, but has discontinuous jumps at "singular set" values $c \in \mathbb{Z}_{\leq 0}$.

Theorem 8.1 (c-Deformed Polylogarithm Ordinary Differential Equation) Let $m \in \mathbb{Z}_{\geq 0}$ and let $c \in \mathbb{C}$ be fixed.

(1) The function $F(z)=L i_{m, c}(z)$ satisfies the ordinary differential equation

$$
D_{m+1}^{c} F(z)=0
$$

where $D_{m+1}^{c} \in \mathbb{C}\left[z, \frac{d}{\mathrm{~d} z}\right]$ is the linear ordinary differerential operator

$$
D_{m+1}^{c}:=z^{2} \frac{d}{\mathrm{~d} z}\left(\frac{1-z}{z}\right)\left(z \frac{d}{\mathrm{~d} z}+c-1\right)^{m}
$$

of order $m+1$.

(2) The operator $D_{m+1}^{c}$ has singular points contained in the set $\{0,1, \infty\}$ on the Riemann sphere in $z$, all of which are regular singular points. In particular, this equation is a Fuchsian operator for all $c \in \mathbb{C}$.

(3) A basis of solutions of $D_{m+1}^{c}$ for $c \in \mathbb{C} \backslash \mathbb{Z}_{\leq 0}$ is, for $z \in \mathbb{C} \backslash\{(-\infty, 0] \cup[1, \infty)\}$, given by

$$
\mathcal{B}_{m+1, c}:=\left\{L i_{m, c}(z), z^{1-c}(\log z)^{m-1}, z^{1-c}(\log z)^{m-2}, \ldots, z^{1-c}\right\}
$$

(For $c \in \mathbb{Z}_{\leq 0}$ the function $L i_{k, c}(z)$ is not well-defined.)

(4) A basis of solutions of $D_{m+1}^{c}$ for $c=-k \in \mathbb{Z}_{\leq 0}$ is, for $z \in \mathbb{C} \backslash\{(-\infty, 0] \cup[1, \infty)\}$, given by

$$
\mathcal{B}_{m+1, c}^{*}:=\left\{L i_{m,-k}^{*}(z), z^{1-c}(\log z)^{m-1}, z^{1-c}(\log z)^{m-2}, \ldots, z^{1-c}\right\}
$$

in which

$$
L i_{m,-k}^{*}(z):=\sum_{\substack{n=0 \\ n \neq k}}^{\infty} \frac{z^{n+1}}{(n-k)^{m}}+\frac{1}{m !} z^{k+1}(\log z)^{m} .
$$

To prove this result, we use a preliminary lemma, concerning the form of the equation. 
Lemma 8.2 The operator

$$
D_{m+1}^{c}=\sum_{k=0}^{m+1} a_{m+1, k}^{c}(z) \frac{d^{k}}{\mathrm{~d} z^{k}}
$$

in which $a_{m+1, k}^{c}(z) \in \mathbb{C}[z]$ is of degree at most $j+1$, with factorization

$$
a_{m+1, k}^{c}(z)=\left(\alpha_{m+1, k}(c) z+\beta_{m+1, k}(c)\right) z^{k}
$$

and the coefficients $\alpha_{m+1, j}(c), \beta_{m+1, j}(c) \in \mathbb{Z}[c]$. The top order coefficient

$$
a_{m+1, m+1}^{c}(z)=(1-z) z^{m}
$$

has coefficients independent of the parameter $c$.

Proof By using the identity $z^{2} \frac{d}{\mathrm{~d} z}\left(\frac{1-z}{z}\right)=(1-z) z \frac{d}{\mathrm{~d} z}-1$ we may rewrite the definition (8.3) as

$$
\begin{aligned}
D_{m+1}^{c} & =\left((1-z) z \frac{d}{\mathrm{~d} z}-1\right)\left(z \frac{d}{\mathrm{~d} z}+c-1\right)^{m} \\
& =(1-z)\left(z \frac{d}{\mathrm{~d} z}+c-1\right)^{m+1}-c\left(z \frac{d}{\mathrm{~d} z}+c-1\right)^{m} .
\end{aligned}
$$

Using the Weyl algebra commutation relation $\frac{d}{\mathrm{~d} z} z=z \frac{d}{\mathrm{~d} z}+1$ we obtain by induction on $j \geq 0$ that

$$
\left(z \frac{d}{\mathrm{~d} z}\right)^{j}=\sum_{k=1}^{j} b_{j, k} z^{k} \frac{d^{k}}{\mathrm{~d} z^{k}}
$$

for certain scalars $b_{j k} \in \mathbb{Z}$, including $b_{j j}=1$. From this we obtain

$$
\left(z \frac{z}{\mathrm{~d} z}+(c-1)\right)^{m}=\sum_{k=0}^{m} f_{m, k}(c) z^{k} \frac{d^{k}}{\mathrm{~d} z^{k}},
$$

in which each $f_{m, k}(c) \in \mathbb{Z}[c]$. Combining this with (8.10) yields

$$
\begin{aligned}
D_{m+1}^{c} & =(1-z)\left(\sum_{k=0}^{m+1} f_{m+1, k}(c) z^{k} \frac{d^{k}}{\mathrm{~d} z^{k}}\right)-c\left(\sum_{k=0}^{m} f_{m, k}(c) z^{k} \frac{d^{k}}{\mathrm{~d} z^{k}}\right) \\
& =\sum_{k=0}^{m+1}\left(-f_{m+1, k}(c) z+f_{m+1, k}(c)-c f_{m, k}(c)\right) z^{k} \frac{d^{k}}{\mathrm{~d} z^{k}} .
\end{aligned}
$$

This yields (8.8) with

$$
\alpha_{m+1, k}(c):=-f_{m+1, k}(c), \quad \beta_{m+1, k}(c):=f_{m+1, k}(c)-c f_{m, k}(c)
$$

and (8.9) follows easily.

Proof of Theorem 8.1. (1) The factor of $z^{2}$ at the front of the differential operator $D_{m+1}^{c}$ is included so that it will have polynomial coefficients and belong to the Weyl algebra $\mathbb{C}\left[z, \frac{d}{\mathrm{~d} z}\right]$, as shown in Lemma 8.2. We check, for $m \geq 1$, 


$$
\left(z \frac{d}{\mathrm{~d} z}+c-1\right) L i_{m, c}(z)=L i_{m-1, c}(z),
$$

where

$$
L i_{0}(c, z)=\sum_{n=1}^{\infty} z^{n}=\frac{z}{1-z}
$$

is independent of $c$. This implies $D_{m+1}^{c} L i_{m, c}(z)=0$.

(2) Using Lemma 8.2 we have

$$
\begin{aligned}
\frac{1}{(1-z) z^{m+1}} D_{m+1}^{c} & =\frac{d^{m+1}}{\mathrm{~d} z^{m+1}}+\sum_{k=0}^{m} \frac{a_{m+1, k}^{c}(z)}{(1-z) z^{m+1}} \frac{d^{k}}{\mathrm{~d} z^{k}} \\
& =\frac{d^{m+1}}{\mathrm{~d} z^{m+1}}+\sum_{k=0}^{m} \frac{\alpha_{m+1, k}(c) z+\beta_{m+1, k}(c)}{(1-z) z^{m+1-j}} \frac{d^{k}}{\mathrm{~d} z^{k}}
\end{aligned}
$$

We now apply standard criteria for identifying singular points and determining if they are regular, given in Coddington and Levinson [14, Chap.4,Theorems5.1,6.1,6.2]. The finite singular points can only be at poles of the coefficients $c_{k}(z)$ of $\frac{1}{(1-z) z^{m+1}} D_{m+1}^{c}=$ $\sum_{k=0}^{m+1} c_{k}(z) \frac{d^{k}}{\mathrm{~d} z^{k}}$, which can occur only at $z=0,1$. The condition for a singular point of first kind (which implies regular singular point) at $z_{0} \in \mathbb{C}$ is that, for $0 \leq j \leq m+1$, the order of the pole at $z_{0}$ of the coefficient $c_{k}(z)$ is at most $m+1-k$. Now (8.12) shows that this condition holds at $z=0,1$. The necessary and sufficient condition for at most a regular singular point at $z=\infty$ is that, for $0 \leq k \leq m+1$, each $c_{k}(z)$ has a zero of order at least $m+1-k$ at $z=\infty$. This clearly holds in (8.12) as well. Thus the singular points are always a subset of $\{0,1, \infty\}$ and each point is either nonsingular or else a regular singular point is regular, for all $c \in \mathbb{C}$. Thus the differential operator is Fuchsian for all $c \in \mathbb{C}$. (In fact $z=1$ is not a singular point for $m=0$ and all $c \in \mathbb{C}$, but when $m \geq 1$ all three points $0,1, \infty$ are genuine singularities for all values of $c$.)

(3) For integer $k \geq 0$ we have

$$
\left(z \frac{d}{\mathrm{~d} z}+c-1\right) z^{1-c}(\log z)^{k}=k z^{1-c}(\log z)^{k-1} .
$$

It follows that $\left\{z^{1-c}(\log z)^{j}: 0 \leq j \leq m-1\right\}$ are annihilated by $\left(z \frac{d}{\mathrm{~d} z}+c-1\right)^{m}$ hence by $D_{m+1}^{c}$. This shows that the $m+1$ functions listed above in $\mathcal{B}_{m+1, c}$ are all in the solution space of $D_{m+1}^{c}$. It remains to check that they are linearly independent over $\mathbb{C}$. The functions $z^{1-c}(\log z)^{k}$ are well-defined solutions for all $c \in \mathbb{C}$, they are linearly independent from powers of the logarithm, and none of them have a singularity at $z=1$. For $c \in \mathbb{C} \backslash \mathbb{Z}_{\leq 0}$ the function $L i_{m, c}(z)$ is a well-defined solution, and it does have a singularity there since it diverges approaching this point along the real axis $z \rightarrow 1^{+}$. We conclude that these $m+1$ functions are a basis of solutions of the differential equation (8.2).

(4) The values $c=-k \in \mathbb{Z}_{\leq 0}$ are singular strata values of the three-variable analytic continuation. To see that $D_{m+1}^{c}\left(L i_{m,-k}^{*}(z)\right)=0$, observe that

$$
\left(z \frac{d}{\mathrm{~d} z}-k-1\right)^{m}\left(\sum_{\substack{n=0 \\ n \neq k}}^{\infty} \frac{z^{n+1}}{(n-k)^{m}}\right)=\sum_{\substack{n=1 \\ n \neq k+1}}^{\infty} z^{n}
$$


while

$$
\left(z \frac{d}{\mathrm{~d} z}-k-1\right)^{m}\left(z^{k+1}(\log z)^{m}\right)=m ! z^{k+1}
$$

Combining these results gives

$$
\left(z \frac{d}{\mathrm{~d} z}-k-1\right)^{m} L i_{m,-k}^{*}(z)=\sum_{n=1}^{\infty} z^{n}=\frac{z}{1-z},
$$

which gives the result. Linear independence over $\mathbb{C}$ is verified as in (3).

We now compute the monodromy representation of this differential equation. We view $L i_{m, c}(z):=z \Phi(m, z, c)$ as a multivalued function of the variable $z$ on the domain $\mathbb{P}^{1}(\mathbb{C}) \backslash\{0,1, \infty\}$. The fundamental group

$$
\mathrm{F}_{2}:=\pi_{1}\left(\mathbb{P}^{1}(\mathbb{C}) \backslash\{0,1, \infty\},-1\right)=\left\langle\left[Z_{0}\right],\left[Z_{1}\right]\right\rangle
$$

is a free group on two generators. It acts on the universal cover $\tilde{\mathbb{C}}_{0,1, \infty}$ by deck transformations. We treat the "non-singular" case that $c \in \mathbb{C} \backslash \mathbb{Z}_{\leq 0}$ and the "singular" case $c \in \mathbb{Z}_{\leq 0}$ separately.

Definition 8.3 Let $\mathcal{W}_{m+1, c}$ denote the $(m+1)$-dimensional complex vector space of functions on $\mathbb{P}^{1}(\mathbb{C}) \backslash\{0,1, \infty\}$ spanned by the vectors in $\mathcal{B}_{m+1, c}$ for $c \in \mathbb{C} \backslash \mathbb{Z}_{\leq 0}$ (resp. $\mathcal{B}_{m+1, c}^{*}$ if $\left.c \in \mathbb{Z}_{\leq 0}\right)$, making cuts along the real axis $(-\infty, 0)$ and $(1, \infty)$. The monodromy representation of $L i_{m, c}(z)$ is the induced action

$$
\rho_{m, c}: \mathrm{F}_{2} \rightarrow \operatorname{Aut}\left(\mathcal{W}_{m+1, c}\right) \simeq G L(m+1, \mathbb{C}) .
$$

The matrix representation on $G L(m+1, \mathbb{C})$ is obtained by viewing the entries of $\mathcal{B}_{m+1, c}$ as a $1 \times(m+1)$ column vector.

We may alternatively view $\mathcal{W}_{m+1, c}$ as a $(m+1)$-dimensional vector bundle over the manifold $\mathcal{X}:=\mathbb{P}^{1}(\mathbb{C}) \backslash\{0,1, \infty\}$.

Theorem 8.4 (c-Deformed Polylogarithm Monodromy-Nonsingular Case) For each integer $m \geq 1$ and each $c \in \mathbb{C} \backslash \mathbb{Z}_{\leq 0}$, the monodromy action on the basis

$$
\mathcal{B}_{m+1, c}:=\left\{L i_{m, c}(z), z^{1-c}(\log z)^{m-1}, z^{1-c}(\log z)^{m-2}, \ldots, z^{1-c}\right\}
$$

is given by

$$
\rho_{m, c}\left(\left[Z_{0}\right]\right):=\left(\begin{array}{cccccc}
1 & 0 & 0 & \cdots & 0 & 0 \\
0 & e^{-2 \pi i c} & \frac{2 \pi i}{1 !} e^{-2 \pi i c} & \cdots & \frac{(2 \pi i)^{m-2}}{(m-2) !} e^{-2 \pi i c} & \frac{(2 \pi i)^{m-1}}{(m-1) !} e^{-2 \pi i c} \\
\vdots & \vdots & \vdots & & \vdots & \vdots \\
0 & 0 & 0 & \cdots & e^{-2 \pi i c} & \frac{2 \pi i}{1 !} e^{-2 \pi i c} \\
0 & 0 & 0 & \cdots & 0 & e^{-2 \pi i c}
\end{array}\right)
$$

and

$$
\rho_{m, c}\left(\left[Z_{1}\right]\right):=\left(\begin{array}{cccccc}
1 & -2 \pi i & 0 & \cdots & 0 & 0 \\
0 & 1 & 0 & \cdots & 0 & 0 \\
\vdots & \vdots & \vdots & \vdots & \vdots & \vdots \\
0 & 0 & 0 & \cdots & 1 & 0 \\
0 & 0 & 0 & \cdots & 0 & 1
\end{array}\right)
$$


The image of $\rho_{m, c}$ falls in a Borel subgroup of $G L(m+1, \mathbb{C})$. The image is unipotent if $c \in \mathbb{Z}_{>1}$, and is quasi-unipotent if $c \in \mathbb{Q} \backslash \mathbb{Z}$.

Proof Around $z=0$ the function $L i_{m, c}(z)$ is analytic, so it has no monodromy. This gives the first row in the matrix (8.14). We also have, for all $c \in \mathbb{C}$,

$$
\begin{aligned}
M_{\left[Z_{0}\right]}\left(\frac{1}{n !} z^{1-c}(\log z)^{n}\right) & =\frac{1}{n !}\left(e^{-2 \pi i(1-c)} z^{1-c}(\log z+2 \pi i)^{n}-z^{1-c}(\log z)^{n}\right) \\
& =e^{-2 \pi i c} z^{1-c}\left(\sum_{j=1}^{n} \frac{1}{n !}\left(\begin{array}{c}
n \\
j
\end{array}\right)(2 \pi i)^{j}(\log z)^{n-j}\right) \\
& =e^{-2 \pi i c} z^{1-c}\left(\sum_{j=1}^{n} \frac{(2 \pi i)^{j}}{j !}\left(\frac{1}{(n-j) !}(\log z)^{n-j}\right)\right) .
\end{aligned}
$$

This gives the remaining rows in (8.14).

Around $z=1$, we note first that the $b_{n, c}(z)=\frac{1}{n !} z^{1-c}(\log z)^{n}$ in $\mathcal{B}_{m+1, c}$ are analytic at $z=1$, which give zero entries above the diagonal in the last $m$ rows of (8.15). It remains to evaluate the monodromy of $L i_{m, c}(z)$. We use the analytic continuation of the Lerch transcendent given in Sect. 3. Note that Theorem 3.4 shows that when $s=m \in \mathbb{Z}$, the monodromy of $\tilde{Z}$ along $\left[Y_{n}\right]$ vanishes for all $n \in \mathbb{Z}$; hence we may view the restricted function $\tilde{Z}^{m}(z, c,[\gamma])$ as a function on $\tilde{\mathbb{C}}_{01 \infty} \times\left(\mathbb{C} \backslash \mathbb{Z}_{\leq 0}\right)$. We denote by $\tilde{Z}_{m, c}(z)$ ( resp. $\left.M_{[\tau]}^{m, c}(\tilde{Z})\right)$ the restrictions of $\tilde{Z}^{m}(z, c,[\gamma])\left(\operatorname{resp} . M_{[\tau]}^{m}(\tilde{Z})\right)$ to $\tilde{\mathbb{C}}_{0,1, \infty} \times\{c\}$. We also treat these as functions of one variable, $z$, on the universal cover $\tilde{\mathbb{C}}_{0,1, \infty}$. By (3.53) we have

$$
M_{\left[Z_{0}\right]^{-k}\left[Z_{1}\right]\left[Z_{0}\right]^{k}}^{m, c}(\tilde{Z})=\alpha(m) f_{k}^{m, c}(z),
$$

in which the constants

$$
\alpha(m):=-\frac{(2 \pi)^{m} e^{\frac{\pi i m}{2}}}{\Gamma(m)}=-\frac{(2 \pi i)^{m}}{(m-1) !}
$$

and the functions

$$
f_{k}^{m, c}(z):=e^{2 \pi i k c} z^{-c}\left(\frac{1}{2 \pi i} \log z-k\right)^{m-1},
$$

are the restrictions of $f_{k}(s, z, c)$ in (3.24) to the domain $\{k\} \times \tilde{\mathbb{C}}_{01 \infty} \times\{c\}$. Choosing $k=0$ yields, for $z$ in the unit interval $(0,1)$,

$$
\begin{aligned}
z M_{\left[Z_{1}\right]}^{m, c}(\tilde{Z}) & =-\frac{(2 \pi i)^{m}}{(m-1) !} e^{-2 \pi i k c} z^{1-c}\left(\frac{1}{2 \pi i \log z}\right)^{m-1} \\
& =-2 \pi i\left(\frac{1}{(m-1) !} e^{-2 \pi i k c} z^{1-c}(\log z)^{m-1}\right)
\end{aligned}
$$

since $\log z=\log z$ on $(0,1)$. This gives the first row in (8.16).

Now we treat the "singular" case $c \in \mathbb{Z}_{\leq 0}$ and determine that the monodromy representation jumps discontinuously between the "nonsingular" and "singular" cases.

Theorem 8.5 (c-Deformed Polylogarithm Monodromy-Singular Case) For each integer $m \geq 1$ and each $c \in \mathbb{Z}_{\leq 0}$, the monodromy action on the modified basis

$$
\mathcal{B}_{m+1, c}^{*}:=\left\{L i_{m, c}^{*}(z), z^{1-c}(\log z)^{m-1}, z^{1-c}(\log z)^{m-2}, \ldots, z^{1-c}\right\}
$$


is given by

$$
\rho_{m, c}\left(\left[Z_{0}\right]\right):=\left(\begin{array}{cccccc}
1 & \frac{2 \pi i}{1 !} & \frac{(2 \pi i)^{2}}{2 !} & \ldots & \frac{(2 \pi i)^{m-1}}{(m-1) !} & \frac{(2 \pi i)^{m}}{m !} \\
0 & 1 & \frac{2 \pi i}{1 !} & \ldots & \frac{(2 \pi i)^{m-2}}{(m-2) !} & \frac{(2 \pi i)^{m-1}}{(m-1) !} \\
\vdots & \vdots & \vdots & & \vdots & \vdots \\
0 & 0 & 0 & \ldots & 1 & \frac{2 \pi i}{1 !} \\
0 & 0 & 0 & \ldots & 0 & 1
\end{array}\right)
$$

and

$$
\rho_{m, c}\left(\left[Z_{1}\right]\right):=\left(\begin{array}{cccccc}
1 & -2 \pi i & 0 & \cdots & 0 & 0 \\
0 & 1 & 0 & \cdots & 0 & 0 \\
\vdots & \vdots & \vdots & \vdots & \vdots & \vdots \\
0 & 0 & 0 & \cdots & 1 & 0 \\
0 & 0 & 0 & \cdots & 0 & 1
\end{array}\right) .
$$

In these cases the image of $\rho_{m, c}$ falls in a unipotent subgroup of $G L(m+1, \mathbb{C})$.

Proof Recall that for $c=-k \in \mathbb{Z}_{\leq 0}$,

$$
L i_{m,-k}^{*}=\sum_{\substack{n=0 \\ n \neq k}}^{\infty} \frac{z^{n+1}}{(n-k)^{m}}+\frac{1}{m !} z^{k+1}(\log z)^{m} .
$$

We compute the monodromy by taking a scaling limit approaching the singular point. Letting $c=-k+\varepsilon$ for positive $\varepsilon$, we assert that

$$
L i_{m,-k}^{*}=\lim _{\varepsilon \rightarrow 0^{+}} L i_{m, c}-\frac{1}{\varepsilon^{m}}\left(\sum_{j=0}^{m-1} \frac{\varepsilon^{j}}{j !} z^{1-c}(\log z)^{j}\right) .
$$

To verify this, we use the expansion

$$
z^{1-c}=z^{k+1} z^{-\varepsilon}=z^{k+1} \sum_{k=0}^{\infty} \frac{(-1)^{k}}{k !}(\varepsilon \log z)^{k} .
$$

Now we assert that the expansion in powers of $\varepsilon$ is

$$
\begin{aligned}
\frac{1}{\varepsilon^{m}}\left(\sum_{j=0}^{m-1} \frac{\varepsilon^{j}}{j !} z^{-\varepsilon}(\log z)^{j}\right) & =\frac{1}{\varepsilon^{m}} \sum_{j=0}^{m-1}\left(\sum_{k=0}^{\infty} \frac{1}{j !} \frac{(-1)^{k}}{k !} \varepsilon^{j+k}(\log z)^{j+k}\right) \\
& =\frac{1}{\varepsilon^{m}}-\frac{1}{m !}(\log z)^{m}+O(\varepsilon) .
\end{aligned}
$$

Here the last step follows using the identities; if $1 \leq n \leq m-1$,

$$
\sum_{\substack{j+k=n \\ 0 \leq j \leq m-1}} \frac{1}{j !} \frac{(-1)^{k}}{k !}=\frac{1}{n !}(1-1)^{n}=0
$$

while if $n=m$,

$$
\sum_{\substack{j+k=m \\ 0 \leq j \leq m-1}} \frac{1}{j !} \frac{(-1)^{k}}{k !}=-\frac{1}{m !} .
$$


This expansion in powers of $\varepsilon$, holding $z$ fixed, gives (8.23).

Now for $z=1$ the right side of (8.23) has no monodromy except that coming from $L i_{m, c}(z)$; hence the limit of the right side yields

$$
M_{\left[Z_{1}\right]}\left(L i_{m,-k}^{*}\right)(z)=-2 \pi i\left(\frac{1}{(m-1) !} z^{k+1}(\log z)^{m}\right) .
$$

The formula (8.22) follows since the other basis elements in $\mathcal{B}_{m+1,-k}^{*}$ are analytic at $z=1$. For $z=0$, we obtain

$$
\begin{aligned}
M_{\left[Z_{0}\right]}\left(L i_{m,-k}^{*}(z)\right)= & \lim _{\varepsilon \rightarrow 0^{+}} M_{\left[Z_{0}\right]}\left(L i_{m,-k+\varepsilon}(z)\right)-\frac{1}{\varepsilon^{m}} z^{k+1}\left(\sum_{j=0}^{m-1} \frac{\varepsilon^{j}}{j !} M_{\left[Z_{0}\right]}\left(z^{\varepsilon}(\log z)^{j}\right)\right) \\
= & -\frac{1}{\varepsilon^{m}} z^{k+1}\left(\sum_{j=0}^{m-1} \frac{\varepsilon^{j}}{j !}\left(e^{-\varepsilon(\log z+2 \pi i)}(\log z+2 \pi i)^{j}-e^{-\varepsilon \log z}(\log z)^{j}\right)\right) \\
= & -\frac{1}{\varepsilon^{m}} z^{k+1} \sum_{j=0}^{m-1}\left(\sum_{k=0}^{\infty} \frac{1}{j !} \frac{(-1)^{k}}{k !} \varepsilon^{j+k}(\log z+2 \pi i)^{j}\right) \\
& +\frac{1}{\varepsilon^{m}} z^{k+1} \sum_{j=0}^{m-1}\left(\sum_{k=0}^{\infty} \frac{1}{j !} \frac{(-1)^{k}}{k !} \varepsilon^{j+k}(\log z)^{j}\right) \\
= & \frac{1}{m !} z^{k+1}\left((\log z+2 \pi i)^{m}-(\log z)^{m}\right)+O(\varepsilon),
\end{aligned}
$$

where we again used (8.24) at the last step. Expanding this term gives the first row in the monodromy matrix (8.21). The remaining rows follow using (8.14).

Remark 8.6 There is an interpretation of the polylogarithm as a variation of Hodge structure over $\mathbb{P}^{1}(\mathbb{C}) \backslash\{0,1, \infty\}$, described in Bloch [11, p. 278]. This property does cannot extend to irrational parameters $c \notin \mathbb{Q}$ because the monodromy is then not quasiunipotent.

Remark 8.7 For $c \in \mathbb{C} \backslash \mathbb{Z}_{\leq 0}$ the vector space $\mathcal{W}_{m+1, c}$ is spanned by the vector space of functions on $\tilde{\mathbb{C}}_{0,1, \infty}$ that is the direct sum of all the images of $L i_{m, c}$ under the action of $\mathrm{F}_{2}$, i.e.

$$
\mathcal{W}_{m+1, c}=\bigoplus_{[\tau] \in \mathrm{F}_{2}} \mathbb{C}\left[Q_{[\tau]}\left(L i_{m, c}\right)\right]
$$

in which $Q_{[\tau]}$ are the operators given in Definition 3.2. Viewed as functions of $z$ on $\tilde{\mathbb{C}}_{01 \infty}$, using (8.16), the vector space spanned by $\left\{z M_{\left[Z_{0}\right]^{-k}\left[Z_{1}\right]\left[Z_{0}\right]^{k}}^{m, c}(\tilde{Z}): k \in \mathbb{Z}\right\}$ is the same vector space as that spanned by $\left\{z f_{k}^{m, c}: k \in \mathbb{Z}\right\}$. By expanding the products $\left(\frac{1}{2 \pi i} \log z-k\right)^{m-1}$ for each $k$ we see that this vector space is spanned by $\left\{z f_{0}^{j, c}: 1 \leq j \leq m\right\}$, or, alternatively, $\left\{z M_{\left[Z_{1}\right]}^{j, c}(\tilde{Z}): 1 \leq j \leq m\right\}$.

Remark 8.8 Comparing the formulas of Theorem 8.5 for $c=1$ with those of D. Ramakrishnan for the monodromy of the polylogarithm, in [67] and [68, Sect. 4.2 and Sect. 7.6], we note some discrepancies. The formulas for $\left[Z_{1}\right]$ given in [68, Sect 4.2] disagree with ours in a sign, and those in [68, Prop. 7.6.7] we believe have a misprint that interchanges $\left[Z_{0}\right]$ and $\left[Z_{1}\right]$. (We think our formulas are correct.) 


\section{Further directions}

There are many directions for further investigation; we discuss a few of them.

(1) Can one better understand the nature of the singularities on the singular strata? In particular, the Riemann zeta function is (formally) obtained as a limit function on a doubly specialized singular stratum. Part I showed that there are obstructions to this limiting process: for example, limiting values approaching the singular stratum $a=1$ in the $(a, c)$-variables exist only for $\operatorname{Re}(s)>1$. It is an interesting problem to obtain "renormalized" limits on singular strata for other ranges of the $s$-variable. In part I [44, Sect. 6] the authors gave a way to do this for the Lerch zeta function for one fixed singular stratum, by removing a small number of divergent terms.

In this paper in Sect. 6 we showed that one can extract data approaching the singular stratrum at $c=0^{+}$and negative integer $s$ sufficient to reconstruct $p$-adic $L$-functions.

(2) The Lerch zeta function possesses additional discrete symmetries. One can define an action of a commuting family of (two-variable) "Hecke operators" in the $(a, c)$ variables on the Lerch zeta function (resp. $(z, c)$-variables for the Lerch transcendent) for which $\zeta(s, a, c)$ (resp. $\Phi(s, z, c))$ is a simultaneous eigenfunction, acting on various function spaces. Part IV ([48]) of this series considers such operators on a function space with real variables. These additional discrete symmetries together with the differential equation (1.12) suggest that there should be an automorphic interpretation of the Lerch zeta function, made in terms of the related functions $L^{ \pm}(s, a, c)$. The first author has found such an interpretation for the real variables form treated in parts I and IV of this series, showing that symmetrized Lerch functions with characters are Eisenstein series on the real Heisenberg group quotiented by the integer Heisenberg group ([45]). It is an open problem to find an automorphic interpretation for the complex-analytic version of the Lerch zeta function (reap. Lerch transcendent) treated in part II and this paper.

(3) One can ask if results of this paper might be interpretable in the framework of an algebraic $D$-module over the Weyl algebra $\mathbb{C}\left[c, z, \frac{\partial}{\partial c}, \frac{\partial}{\partial z}\right]$, which is associated to a parametric family of linear PDE's $\Delta_{\Phi}-s I$ with

$$
\Delta_{\Phi}:=\frac{1}{2}\left(\mathrm{D}_{\Phi}^{+} \mathrm{D}_{\Phi}^{-}+\mathrm{D}_{\Phi}^{-} \mathrm{D}_{\Phi}^{+}\right)=z \frac{\partial}{\partial z} \frac{\partial}{\partial c}+c \frac{\partial}{\partial c}+\frac{1}{2} I,
$$

with $s$ as eigenvalue parameter. Such a reformulation may involve a non-holonomic $D$-module with an infinite set of singularities.

(4) What are the properties of the extension of polylogarithms under deformation in the $c$-variable? As mentioned in Sect. 1.3, one can ask whether functional equations such as the five-term relation for the dilogarithm might survive in some fashion under the $c$-deformation of the polylogarithm studied in Sect. 8. Specifically, the integer points $c=m \geq 2$ and $s=n \geq 1$ have maximally unipotent monodromy with apparent discontinuity in the monodromy matrices. One can ask whether the functions at these special points satisfy interesting identities in parallel fashion to the polylogarithms.

The dilogarithm is known to have a single-valued variant, the Rogers dilogarithm, obtained by adding a correction term. One may wonder if there exists analogous single-valued variant of the extended function in the $c$-variable, or at specific integer points $c=m \geq 2$. 
(5) One may investigate generalizations of the Lerch transcendent in the direction of an "elliptic Lerch zeta function", made in analogy with work of Beilinson [6] and Levin [50]) on the elliptic polylogarithm.

(6) The partial differential operator $\Delta_{\Phi}$ in (1.12) in the introduction can be viewed as an unbounded operator acting on functions restricted to the domain

$$
T:=\left\{(z, c) \in S^{1} \times[0,1]\right\}, \quad \text { with } \quad S^{1}=\{|z|=1\},
$$

inside the Hilbert space $L^{2}\left(T, \frac{d z}{z} d c\right)$. On this Hilbert space $\Delta_{\Phi}$ can be shown to be formally skew-adjoint and to have the $x p$-form suggested by Berry and Keating $([8,9])$ as the possible form of a Hilbert-Polya operator encoding the zeta zeros as eigenvalues.

This operator is obtained from the corresponding operator $D_{L}=\frac{1}{2 \pi i} \frac{\partial}{\partial a} \frac{\partial}{\partial c}+c \frac{\partial}{\partial c}+\frac{1}{2}$ for the Lerch zeta function, which satisfies

$$
D_{L}(s, a, c)=-\left(s-\frac{1}{2}\right) \zeta(s, a, c) .
$$

acting on the Hilbert space $L^{2}\left([0,1]^{2}, d a d c\right)$, which is treated in [47, Sect. 9.2].

One may search for natural skew-adjoint "boundary conditions" on the operator $\Delta_{\Phi}$ of $D_{L}$ which yield operators having spectra on the line $\mathfrak{R}\left(s-\frac{1}{2}\right)=0$. One such set of boundary conditions will be presented in [45, Sect. 9]; the spectrum of the resulting operator is purely continuous. It is an open question whether one can formulate natural geometric boundary conditions on $\Delta_{\Phi}$ that will yield a Hilbert-Polya operator for $\zeta(s)$.

\footnotetext{
Author details

'Department of Mathematics, University of Michigan, Ann Arbor, MI 48109-1043, USA, ${ }^{2}$ Department of Mathematics, Pennsylvania State University, University Park, PA 16802-8401, USA.
}

\section{Acknowledgements}

The authors thank Dinakar Ramakrishnan for conversations regarding his work on polylogarithms. The first author thanks Peter Scott for discussions and queries on multidimensional covering manifolds. The authors thank the reviewers for helpful comments. This project was initiated while the first author was at AT\&T Labs-Research and the second author consulted there; they thank AT\&T for support. The first author received support from the Mathematics Research Center at Stanford University in 2009-2010. The second author received support from the National Center for Theoretical Sciences and National Tsing Hua University in Taiwan in 2009-2014. To these institutions the authors express their gratitude. The research of the first author was supported by NSF grants DMS-1101373 and DMS-1401224 and that of the second author by NSF-grant DMS-1101368 and Simons Foundation grant No. 355798

Received: 20 June 2015 Accepted: 20 November 2015

Published online: 25 February 2016

\section{References}

1. Amice, Y., Fresnel, J.: Fonctions zeta p-adiques des corps de nombres abéliens réels. Acta Arith. 20, $353-384$ (1972)

2. Apostol, T.M.: On the Lerch zeta function. Pacific J. Math. 1, 161-167 (1951)

3. Apostol, T.M.: Introduction to Analytic Number Theory. Springer-Verlag, New York (1976)

4. Barnes, E.W.: On certain functions defined by Taylor's series with finite radius of convergence. Proc. Lond. Math. Soc. (Ser. 2) 4, 287-316 (1906)

5. Beilinson, A.: Higher regulators and the values of L-functions, (Russian) Current problems in mathematics, vol. 24, pp. 181-238 Itogi Nauki i Tekhiki, Moscow 1984. (English Translation: J. Soviet Math. 30 (1985), 2036-2070.)

6. Beilinson, A., Deligne, P.: Interprétation motivique de la conjecture de Zagier reliant polylogarithmes et régulateurs., in: Motives, (Seattle, Wash. 1991), 97-121, Proc. Symp. Pure Math. Vol. 55 , No. 2, American Mathematical Society, Providence (1994)

7. Beilinson, A., Levin, A.: The elliptic polylogarithm. In: Motives (Seattle, WA 1991), 123-190, Proc. Symp. Pure Math. 55 Part 2, American Mathematical Society: Providence (1994)

8. Berry, M.V., Keating, J.P.: H = xp and the Riemann zeros. In: Lerner, I.V., Keating, J.P., Khmelnitskii, D.E. (eds.) Supersymmetry and Trace Formulae: Chaos and Disorder. NATO ASI Series B: Physics No. B370, pp. 355-367. Kluwer Academic, New York (1999)

9. Berry, M.V., Keating, J.P.: The Riemann zeros and eigenvalue asymptotics. SIAM Rev. 41, 236-266 (1999)

10. Besser, A.: Finite and p-adic polylogarithms: Compositio Math. 130(2), 215-223 (2002)

11. Bloch, S.: Function theory of polylogarithms. In: Lewin, L. (ed.) Structural Properties of Polylogarithms, pp. $275-286$. American Mathematical Society, Providence (1991) 
12. Cartier, P.: Fonctions polylogaithmes, nombres polyzetas et groupes pro-unipotents, Séminarire Bourbaki, vol. 2000/2001, Astérisque No. 282 (2002), Exp. No. 885, pp. 137-173

13. Chakraborty, K., Kanemitsu, S., Tsukada, H.: Vistas of Special Functions II. World Scientific Publ. Co., Singapore (2010)

14. Coddington, E.A., Levinson, N.: Theory of Ordinary Differential Equations. McGraw-Hill Book Co., New York (1955)

15. Coleman, R.F.: Dilogarithms, regulators, and p-adic L-functions. Invent. Math. 69, 171-208 (1982)

16. Costin, O., Garoufalidis, S.: Resurgence of the Euler-MacLaurin summation formula. Ann. Inst. Fourier (Grenoble) 58(3), 893-914 (2008)

17. Costin, O., Garoufalidis, S.: Resurgence of the fractional polylogarithms. Math. Res. Lett. 16, 817-826 (2009)

18. Deligne, P.: Le groupe fondamental de la droite projective moins trois points. In: Ihara, Y., Ribet, K., Serre, J.-P. (eds.) Galois Groups over Q, pp. 79-297. New York, Springer-Verlag (1989)

19. Derek, J.S.: Robinson, A Course in the Theory of Groups. Springer-Verlag, New York (1982)

20. Dupont, J.L.: The dilogarithm as a characteristic class for flat bundles, Proc. of Northwestern Conference on cohomology of groups (Evanston III. 1985), J. Pure Appl. Alg. 44(1-3), 137-164 (1987)

21. Erdélyi, A., Magnus, W., Oberhettinger, F., Tricomi, F.: Higher transcendental functions. Based in part on notes left by Harry Bateman, vol. I. McGraw-Hill, New York (1953)

22. Euler, L.: De summatione serierum in hac forma contentarum $\frac{a}{1}+\frac{a^{2}}{4}+\frac{a^{3}}{9}+\frac{a^{4}}{16}+\frac{a^{5}}{25}+\frac{a^{6}}{36}+$ etc., Memoires de l'acadmie des sciences de St.-Petersbourg 3, 26-42 (1811). (Opera Omnia, Series 1, Vol. 16, pp. 117-138) [Enëstrom Catalogue: E736]

23. Faddeev, L.D., Kashaev, R.M.: Quantum dilogarithm. Mod. Phys. Lett. A 9(5), 427-434 (1994)

24. Furusho, H.: p-adic multiple zeta values I. p-adic multiple polylogarithms and the p-adic KZ equation. Invent. Math. 155, 253-286 (2004)

25. Furusho, H.: p-adic multiple zeta values II. Tannakian interpretations. Am. J. Math. 129(4), 1105-1144 (2007)

26. Garunkštis, R., Laurenčikas, A.: On one Hilbert's problem for the Lerch zeta function. Publ. Inst. Math. (Beograd) 65 (79), 63-68 (1999)

27. Gel'fand, I.M., MacPherson, R.D.: Geometry in Grassmannians and a generalization of the dilogarithm. Adv. Math. 44(3), 279-312 (1982)

28. Goncharov, A.B.: Polylogarithms and motivic Galois groups. In: Motives (Seattle, WA 1991), 43-96, Proc. Symp. Pure Math. 55, Part 2, American Mathematical Society, Providence (1994)

29. Goncharov, A.B.: Geometry of configurations, polylogarithms and motivic cohomology. Adv. Math. 114, 197-318 (1995)

30. Guillera, J., Sondow, J.: Double integrals and infinite products for some classical constants via analytic continuations of Lerch's transcendent. Ramanujan J. 16, 247-270 (2008)

31. Hain, R.: Classical polylogarithms. In: Motives (Seattle, WA 1991). 3-42, Proc. Symp. Pure Math. 55, Part 2, AMS, Providence (1994)

32. Hain, R., MacPherson, R.: Higher logarithms. III. J. Math. 34(2), 392-475 (1990)

33. Hatcher, A.: Algebraic Topology. Cambridge University Press, Cambridge (2001)

34. Hilbert, D.: Mathematische Probleme, Göttinger Nachrichten (1900) 253-297. Reprinted in: Archiv der Mathematik und Physik, 3rd Ser. 1, 44-63 and 213-237 (1901). [English translation: Mathematical Problems, Bull. Amer. Math. Soc. 8 (1902) 437-479. Reprinted in: Mathematical Developments Arising From Hilbert Problems, Proc. Symp. Pure Math. Volume 28, AMS: Providence 1976, pp. 1-34.]

35. Huber, A., Wildeshaus, J.: Classical motivic polylogarithm according to Beilinson and Deligne, Doc. Math. 3, 27-133 (1998). [Correction: 3 (1998), 197-199]

36. Jonquière, A.: Note sur la série $\sum_{n=1}^{\infty} \frac{x^{n}}{n^{5}}$. Bull. Soc. Math. France 17, 142-152 (1889)

37. Joyner, S.E.: On an extension of the universal monodromy representation for $P^{1}\{0,1, \infty\}$, Comm. Number Theory Phys. 8(3), 369-402 (2014)

38. Kanemitsu, S., Katsurada, M., Yoshimoto, M.: On the Hurwitz-Lerch zeta-function. Aequ. Math. 59, 1-19 (2000)

39. Kanemitsu, S., Tsukada, H.: Vistas of Special Functions. World Scientific, Singapore (2007)

40. Kashaev, R.M., Nakanishi, T.: Classical and quantum dilogarithm identities. SIGMA 7(102), 29 (2011)

41. Kirillov, A.N.: Dilogarithm identities, partitions, and spectra in conformal field theory, Algebra i Analiz 6(2), 152-175 (1994), translation: St. Petersburg Math. J. 6 (1995), no. 2, 327-348

42. Kirillov, A.N.: Dilogarithm identities. In: Quantum field theory, integrable models and beyond (Kyoto 1994), Prog. Theor. Phys. Suppl. No. 118, pp. 61-142 (1995)

43. Kubota, T., Leopoldt, H.W.: Eine p-adische Theorie der Zetawerte I. J. Reine Angew. 214(215), 328-339 (1964)

44. Lagarias, J.C.: Euler's constant: Euler's work and modern developments. Bull. Amer. Math. Soc. N. S. 50(4), 527-628 (2013)

45. Lagarias, J.C.: The Lerch zeta function and the Heisenberg group, eprint: arXiv:1511.08157

46. Lagarias, J.C., Li, W.-C.W.: The Lerch zeta function I. Zeta integrals. Forum Math. 24, 1-48 (2012)

47. Lagarias, J.C., Li, W-C.W.: The Lerch zeta function II. Analytic continuation, Forum Math. 24, 49-84 (2012)

48. Lagarias, J.C., Li, W-C.W.: The Lerch zeta function IV. Hecke operators, eprint: arxiv:1511.08116

49. Laurenčikas, A., Garunkštis, R.: The Lerch zeta-function. Kluwer Academic Publishers, Dordrecht (2002)

50. Lerch, M.: Note sur la fonction $\mathcal{R}(w, x, s)=\sum_{k=0}^{\infty} \frac{e^{2 k \pi i x}}{(w+k)^{s}}$. Acta Math. 11, 19-24 (1887)

51. Levin, A.: Elliptic polylogarithms: an analytic theory. Compos. Math. 106, 267-282 (1997)

52. Lewin, L.: Polyalgorithms and Associated Functions. North-Holland, New York (1981)

53. Lewin, L.: The Evolution of the Ladder Concept, pp. 1-10. In: Lewin, L. (ed.) Structural Properties of Polylogarithms, AMS, Providence (1991)

54. Lewin, L. (ed.): Structural Properties of Polylogarithms. AMS, Providence (1991)

55. Mihh, H.N., Petitot, M., van der Hoeven, J.: Shuffle algebra and polylogarithms. Discret. Math. 225(1-3), $217-230$ (2000)

56. Morita, Y.: On the Hurwitz-Lerch L-functions. J. Fac. Sci. Univ. Tokyo 14, 29-43 (1977)

57. Mumford, D.: Tata Lectures on Theta I. Birkhäuser, Boston (1983) 
58. Nahm, W., Recknagel, A., Terhoeven, M.: Dilogarithm identities in quantum field theory. Mod. Phys. Lett. A 8, 1835-1847 (1993)

59. Naito, H.: The $p$-adic Hurwitz L functions. Tohoku Math. J. 34, 553-558 (1982)

60. Nakamura, H., Wojtkowiak, Z:: On explicit formulae for $\ell$-adic polylogarithms. In: Arithmetic fundamental groups and noncommutative algebra (Berkeley, CA 1999), 285-294, Proc. Symp. Pure Math. 70, American Mathematical Society, Providence (2002)

61. Neumann, W.D.: Extended Bloch group and the Cheeger-Cherns-Simons class. Geom. Topol. 8, 413-474 (2004)

62. Oberhettinger, E.: Note on the Lerch zeta function. Pacific J. Math. 6, 117-120 (1956)

63. Oesterlé J.: Polylogarithmes, Séminare Bourbaki, 35, Exposé No. 762, pp. 19 (1992-1993)

64. Ostrowski, A.: Über Dirichletsche Reihen und algebraischen Differentialgleichungen. Math. Z. 8, 241-298 (1920)

65. Patterson, S.J.: An introduction to the theory of the Riemann zeta function. Cambridge University Press, Cambridge (1988)

66. Ramakrishnan, D.: A regulator for curves via the Heisenberg group. Bull. Amer. Math. Soc. N. S. 5(2), 191-195 (1981)

67. Ramakrishnan, D.: On the monodromy of higher logarithms. Proc. Amer. Math. Soc. 85, 596-599 (1982)

68. Ramakrishnan, D.: Regulators, Algebraic Cycles, and Values of L-Functions. In: Algebraic K-Theory and algebraic number theory (Honolulu, HI 1987), Contemporary Math. vol. 83, pp. 183-310 (1989)

69. Rogers, L.J.: On function sum theorems connected with the series $\sum_{1}^{\infty} \frac{x^{n}}{n^{2}}$. Proc. London Math. Soc. 4, 169-189 (1907)

70. Spence, W.: An essay on logarithmic transcendents. Edinburgh, London (1809)

71. Srivastava, H.M., Choi, J.: Series Associated with the Zeta and Related Functions. Kluwer Academic Publishers, Dordrecht (2001)

72. Vepstas, L: An efficient algorithm for accelerating the convergence of oscillatory series, useful for computing the polylogarithm and Hurwitz zeta functions. Numer. Algorithms 47(3), 211-252 (2008)

73. Washington, L.C.: Introduction to Cyclotomic Fields, 2nd edn. Springer-Verlag, New York (1997)

74. Weil, A.: Elliptic Functions according to Eisenstein and Kronecker. Springer-Verlag, New York (1976)

75. Whittaker, E.T., Watson, G.N.: A Course of Modern Analysis, 4th edn. Cambridge University Press, Cambridge (1927). (Reprint 1965)

76. Wildeshaus, J.: Realizations of polylogarithms. Lecture Notes in Mathematics, vol. 1650. Springer-Verlag, Berlin (1997)

77. Wojtkowiak, Z: On $\ell$-adic interated integrals I, Analog of Zagier conjecture. Nagoya Math. J. 176, 113-158 (2004)

78. Wojtkowiak, Z.: On $\ell$-adic interated integrals II. Functional equations and $\ell$-adic polylogarithms. Nagoya Math. J. 177, 117-153 (2005)

79. Wojtkowiak, Z:: On $\ell$-adic interated integrals III. Galois actions on fundamental groups. Nagoya Math. J. 178, 1-36 (2005)

80. Zagier, D.: The dilogarithm function in geometry and number theory. In: Number Theory and Related Topics (Bombay 1988), 231-249, Tata Inst. Fund. Res. Stud. Math. 12, Tata Institute, Bombay (1989) (Also: The remarkable dilogarithm, J. Math. Phys. Sci. 22 (1988), no. 1, 131-145.)

81. Zagier, D.: The dilogarithm function, pp. 3-65. In: Frontiers in number theory, physics, and geometry, Vol II, Springer, Berline (2007)

\section{Submit your manuscript to a SpringerOpen ${ }^{\circ}$ journal and benefit from:}

- Convenient online submission

- Rigorous peer review

- Immediate publication on acceptance

- Open access: articles freely available online

- High visibility within the field

- Retaining the copyright to your article

Submit your next manuscript at $\boldsymbol{s p r i n g e r o p e n . c o m ~}$ 